\title{
ON SOME APPLICATIONS OF THE UNIVERSAL ENVELOPING ALGEBRA OF A SEMISIMPLE LIE ALGEBRA
}

\author{
BY \\ HARISH-CHANDRA $(1)$
}

Introduction. The representation theory of semisimple Lie algebras over the field of complex numbers has been developed by Cartan and Weyl. However some of Cartan's proofs (see [2])( $\left.{ }^{2}\right)$ make explicit use of the classification of semisimple Lie algebras and in fact require a verification of the asserted statement in each case separately. Weyl [12] has given alternative proofs of these results by making use of general arguments depending on the theory of representations of compact groups and in particular on the PeterWeyl Theorem. His proofs therefore are necessarily of a nonalgebraic nature. In the first part of this paper we propose to give "general" algebraic proofs of some of these theorems. This work happens to overlap considerably with some recent results of Chevalley [3]. In particular the formulation of Theorem 1 and some of the ideas in the proof are due to him. I shall mention them more specifically later in due course.

Recently great interest has arisen in the theory of representations of a Lie group in a Hilbert space. Since every such representation defines a representation of the corresponding Lie algebra (see Gårding [8]) it is natural to study infinite-dimensional representations of a Lie algebra. Part II of this paper contains a theorem (Theorem 4) concerning such representations of complex semisimple Lie algebras. The desirability of proving such a result was pointed out to me by Mautner. Also its significance for unitary representations of complex semisimple Lie groups on a Hilbert space will be brought out by him in a separate paper.

In Part III we define and study the characters of the universal enveloping algebra $\mathfrak{B}$ of a semisimple Lie algebra $\mathfrak{R}$. They are essentially homomorphisms of the center of $\mathfrak{B}$ into the field of complex numbers. We show that every such homomorphism is determined by a linear function on a fixed Cartan subalgebra of $\mathbb{R}$. Theorems 5 and 6 contain the principal results of Part III.

Part IV is devoted to a brief study of the representations of a complex semisimple Lie group on a Hilbert space. With certain representations of the group (in particular with all those which are irreducible and unitary) we associate in a natural way a character of $\mathfrak{B}$. It follows from Theorem 6 that in order that a character may be associated to some representation of the

Received by the editors March 27, 1950.

(1) Frank B. Jewett Fellow.

(2) Numbers in brackets refer to the references at the end of the paper. 
group it must satisfy certain conditions. By a method due to Gelfand and Naimark [7] we show in Theorem 7 that these conditions are also sufficient.

I should like to thank Dr. F. I. Mautner for a number of very valuable discussions and also for his help in some questions concerning operator theory which arise in Part IV.

Part I. Representations of semisimple Lie algebras. Let $\mathfrak{l}$ be a semisimple Lie algebra over an algebraically closed field $K$ of characteristic zero. For any $X \in \mathbb{R}$ let ad $X$ denote the linear mapping $(\operatorname{ad} X) Y=[X, Y](Y \in \mathfrak{R})$ of $\mathfrak{R}$ into itself. Put $B(X, Y)=\operatorname{sp}(\operatorname{ad} X$ ad $Y)$. Then $B(X, Y)$ is a nondegenerate bilinear form defined on $\mathfrak{R}$. Let $\mathfrak{h}$ be a Cartan subalgebra of $\mathfrak{l}$ and $\alpha$ a root of $\mathfrak{Q}$ with respect to $\mathfrak{h}$. We denote by $H_{\alpha}^{\prime}$ the unique element in $\mathfrak{h}$ such that $B\left(H_{\alpha}^{\prime}, H\right)=\alpha(H)$ for all $H \in \mathfrak{h}$. It is known that $\alpha\left(H_{\alpha}^{\prime}\right)$ is a rational number greater than 0 . Put $H_{\alpha}=\left(2 / \alpha\left(H_{\alpha}^{\prime}\right)\right) H_{\alpha}^{\prime}$ so that $\alpha\left(H_{\alpha}\right)=2$ and let $\mathfrak{F}$ be the set of all linear functions on $\mathfrak{h}$ with values in $K$. Then $\mathfrak{F}$ is a vector space over $K$ dual to $\mathfrak{h}$. The linear transformation $s_{\alpha}$ in $\mathfrak{F}$ defined by $s_{\alpha} \lambda=\lambda-\lambda\left(H_{\alpha}\right) \alpha(\lambda \in \mathfrak{F})$ is called the Weyl reflexion with respect to the root $\alpha$. It is known that the group $W$ generated by the $s_{\alpha}$ 's for the various roots $\alpha$ is finite, and if $\alpha$ and $\beta$ are any two roots then $s_{\alpha} \beta$ is also a root. $W$ is called the Weyl group of $\mathcal{R}$ (with respect to $\mathfrak{h})$.

Let $\Sigma=\left\{\alpha_{i}, 1 \leqq i \leqq l\right\}(l=\operatorname{dim} \mathfrak{h})$ be a maximal set of linearly independent roots. We shall say that $\Sigma$ is a fundamental system of roots if every root $\alpha$ is of the form $\alpha=\sum_{1 \leqq i \leqq l} d_{i} \alpha_{i}$ where $d_{i}$ are integers which are either all nonnegative or all nonpositive. It is known that fundamental systems always exist. Further if $\Sigma=\left\{\alpha_{i}, 1 \leqq i \leqq l\right\}$ is a fundamental system then the Weyl reflexions $s_{\alpha_{i}}, 1 \leqq i \leqq l$, generate the whole group $W$ and every root $\alpha$ can be written in the form $\alpha=\sigma \alpha_{i}\left(\sigma \in W, \alpha_{i} \in \Sigma\right)$. Put $s_{i}=s_{\alpha_{i}}$ and $H_{\alpha_{i}}$ $=H_{i}, 1 \leqq i \leqq l$. Then $s_{i} \alpha_{j}=\alpha_{j}+a_{j i} \alpha_{i}$ where $a_{j i}=-\alpha_{j}\left(H_{i}\right)$. Since $s_{i} \alpha_{j}$ is a root, $a_{j i}$ is an integer such that $a_{i i}=-2, a_{i j} \geqq 0, i \neq j$. It is clear that if the integers $a_{i j}, 1 \leqq i, j \leqq l$, are given we can find out which linear combinations of $\alpha_{i}$ are roots since they are all of the form $s_{i_{1}} s_{i_{2}} \cdots s_{i_{r}}, \alpha_{j} 1 \leqq i_{1}, \cdots, i_{r}, j \leqq l, r \geqq 0$. Thus the matrix $A=\left(a_{i j}\right), 1 \leqq i, j \leqq l$ determines the root diagram of $\mathbb{R}$ completely. We shall call $A$ the Weyl matrix of $\mathfrak{R}$ (with respect to $\mathfrak{h}$ ). Notice that $A$ has the following three properties.

$$
\begin{aligned}
a_{i i} & =-2, \quad a_{i j} \geqq 0, \quad i \neq j, \\
a_{i j} & =a_{j i}, \\
\operatorname{det} A & \neq 0 .
\end{aligned}
$$

The last assertion follows from the fact that $\alpha_{i}, 1 \leqq i \leqq l$, are linearly independent. A natural question to ask is the following: Given a square matrix $A$ with integral coefficients what are the conditions which $A$ must satisfy in order that $A$ be the Weyl matrix of some semisimple Lie algebra 8 ? This is one of the two questions considered in Theorem 1. 
Now we come to the second question. Let us call an element $\lambda \in \mathfrak{F}$ rational if $\lambda\left(H_{i}\right) \in K_{0}, 1 \leqq i \leqq l$, where $K_{0}$ is the prime field of characteristic zero contained in $K$. It is clear that $\lambda=\sum_{1 \leqq i \leqq l} c_{i} \alpha_{i}\left(c_{i} \in K_{0}\right)$. We say that $\lambda>0$ if $\lambda \neq 0$ and $c_{i}>0, i$ being the least index such that $c_{i} \neq 0$. Let $\mathfrak{F}_{0}$ be the set of all rational linear functions on $\mathfrak{h}$. For any $\lambda, \mu \in \mathfrak{F}_{0}$ we write $\lambda>\mu$ or $\mu<\lambda$ if $\lambda-\mu>0$. In this way $\mathfrak{F}_{0}$ is completely ordered under the relation $>$. This order is called the lexicographic order in $\mathfrak{F}_{0}$ with respect to the ordered set $\left\{\alpha_{1}, \cdots, \alpha_{l}\right\}$. We shall call an element $\lambda \in \mathfrak{F}_{0}$ integral if $\lambda\left(H_{i}\right)$ is an integer for all $1 \leqq i \leqq l$, and dominant integral if, in addition, $\lambda\left(H_{i}\right) \geqq 0,1 \leqq i \leqq l$. Let $\rho$ be a representation of $\mathfrak{R}$ on a finite-dimensional space $V$. Given any $\lambda \in \mathfrak{F}$ we define $V_{\lambda}$ to be the set of all $\psi \in V$ such that $\rho(H) \psi=\lambda(H) \psi$ for all $H \in \mathfrak{h}$. $\lambda$ is called a weight of $\rho$ if $V_{\lambda} \neq\{0\}$. It is known that every weight $\Lambda$ of $\rho$ is an integral linear function on $\mathfrak{h}$ and $V=\sum_{\boldsymbol{\Lambda}} V_{\boldsymbol{\Delta}}$ where the sum is direct and $\Lambda$ runs over all the weights of the representation, these being only finite in number. Hence $\rho$ has a highest weight $\Lambda_{0}$ and it is known that $\Lambda_{0}\left(H_{i}\right) \geqq 0$, $1 \leqq i \leqq l$, so that $\Lambda_{0}$ is a dominant integral function. The second question can now be phrased as follows: Given a dominant integral function $\Lambda$ does there exist a finite-dimensional representation $\rho$ of $\&$ such that $\Lambda$ is the highest weight of $\rho$ ?

I should like to mention that in my original proof I had considered the second question alone. The idea of dealing with both questions simultaneously is due to Chevalley [3] who obtained independently a proof of the theorem given below. I present here a modified version of my original proof so as to be able to consider the two questions together. But in this modification I have adopted several of Chevalley's ideas. In particular the construction of the algebra $\mathfrak{A}$ and the consideration of its representations on $\mathcal{B}$ is due to him.

THEOREM 1. Let $a_{i j}, 1 \leqq i, j \leqq l$, be $l^{2}$ integers such that:

(1) $a_{i i}=-2, a_{i j} \geqq 0, i \neq j$, and $a_{i j}=0$ whenever $a_{j i}=0,1 \leqq i, j \leqq l$.

(2) $\operatorname{det}\left(a_{i j}\right) \neq 0$.

(3) The group $W$ generated by the linear transformations $s_{i}, 1 \leqq i \leqq l$, given by $s_{i} x_{j}=x_{j}+a_{j i} x_{i}\left(x_{i}, 1 \leqq i \leqq l\right.$, being indeterminates $)$ is finite.

Then there exists a semisimple Lie algebra $\mathbb{Q}$ over $K$ with a Cartan subalgebra $\mathfrak{h}$ such that the following conditions are fulfilled. It is possible to find a set of linear functions $\alpha_{i}, 1 \leqq i \leqq l$, on $\mathfrak{h}$ such that $a_{i}, 1 \leqq i \leqq l$, is a fundamental system of roots of $\mathfrak{R}$ with respect to $\mathfrak{h}$ and $\sigma_{i} \alpha_{j}=\alpha_{j}+a_{j i} \alpha_{i}$ where $\sigma_{i}$ is the Weyl reflexion with respect to $\alpha_{i}$. Finally if $\lambda_{i}, 1 \leqq i \leqq l$, are any given integers greater than or equal to 0 we can find an irreducible finite-dimensional representation $\rho$ of $\mathbb{R}$ such that the highest weight $\Lambda_{0}$ of $\rho$ is given by $\left.{ }^{3}\right) \Lambda_{0}\left(H_{\alpha_{i}}\right)=\lambda_{i}, 1 \leqq i \leqq l$.

Before proceeding with the proof we make some remarks about terminology. All vector spaces and algebras appearing in our discussion are understood to be over $K$. A vector space $V$ (or a representation) is not necessarily

(3) As before we define $H_{\alpha}$ for any root $\alpha$ of $\&$ in such a way that $\alpha\left(H_{\alpha}\right)=2$. 
assumed to be finite-dimensional unless it is explicitly stated to be so. Given any collection $\left\{U_{j} ; j \in I\right\}$ of subspaces of $V$ indexed by a set $I$ (finite or infinite) we denote by $\sum_{j \in I} U_{j}$ the smallest subspace of $V$ containing all $U_{j}$. In fact $\sum_{j} U_{j}$ consists of all finite sums of the form $\psi_{1}+\cdots+\psi_{r}$ where each $\psi_{i}$ belongs to some $U_{j}$. The sum $\sum_{j \in I} U_{j}$ is said to be direct if for every finite subset $I_{0}$ of $I$ the sum $\sum_{j \in I_{0}} U_{j}$ is direct. If $\Sigma$ is any set of linear mappings of $V$ into itself we say that $V$ is irreducible under $\Sigma$ if there exists no subspace $U$ of $V$ such that $U$ is invariant under $\Sigma$ and $U \neq V, U \neq\{0\}$.

Given an associative algebra $\mathfrak{A}$, we shall always write $[z, w]=z w-w z$ for any $z, w \in \mathfrak{A}$. We use a similar notation whenever $z$ and $w$ are matrices or linear transformations on a vector space. If $\mathfrak{M}$ is a left ideal in $\mathfrak{A}$ we define the natural representation $\pi$ of $\mathfrak{A}$ on the factor space $\mathfrak{A}^{*}=\mathfrak{A} / \mathfrak{M}$ as follows. Let $z \rightarrow z^{*}$ denote the natural mapping of $\mathfrak{A}$ on $\mathfrak{A}^{*}$. Then $\pi(z) w^{*}=(z w)^{*}$, $z, w \in \mathfrak{A}$. From the fact that $\mathfrak{M}$ is a left ideal it is easily verified that $\pi$ is a representation.

After these preliminary remarks we return to Theorem 1 . The proof is rather long but is otherwise not very complicated. It depends on the consideration of the representations of a certain infinite-dimensional associative algebra $\mathfrak{A}$. We shall have to prove a series of lemmas about left ideals in this algebra, some of which are very simple but are nevertheless essential. Let $\mathfrak{A}^{\prime}$ be the free associative algebra of all noncommutative polynomials in $3 l$ independent variables $x_{i}^{\prime}, y_{i}^{\prime}, H_{i}^{\prime}, 1 \leqq i \leqq l$, with coefficients in $K$. Let $\mathfrak{h}^{\prime}$ be the subspace of $\mathfrak{A}^{\prime}$ spanned by $H_{i}^{\prime}, 1 \leqq i \leqq l$. We define the linear functions $\alpha_{i}, 1 \leqq i \leqq l$, on $\mathfrak{h}^{\prime}$ by $\alpha_{i}\left(H_{j}^{\prime}\right)=-a_{i j}, i \leqq i, j \leqq l$. Since det $\left(a_{i j}\right) \neq 0, \alpha_{i}$ are linearly independent. Let $\mathfrak{U}^{\prime}$ be the smallest ideal in $\mathfrak{U}^{\prime}$ containing the set $\Im$ consisting of the following elements.

$$
\begin{array}{lr}
{\left[H_{i}^{\prime}, H_{j}^{\prime}\right], \quad\left[X_{i}^{\prime}, Y_{i}^{\prime}\right]-H_{i}^{\prime}, \quad\left[X_{i}^{\prime}, Y_{j}^{\prime}\right],} & i \neq j, \\
{\left[H_{j}^{\prime}, X_{i}^{\prime}\right]-\alpha_{i}\left(H_{j}^{\prime}\right) X_{i}^{\prime}, \quad\left[H_{j}^{\prime}, Y_{i}^{\prime}\right]+\alpha_{i}\left(H_{j}^{\prime}\right) Y_{i}^{\prime},} & 1 \leqq i, j \leqq l .
\end{array}
$$

Let $(\xi)$ be the free associative algebra over $K$ with $l$ generators $\eta_{1}, \cdots, \eta_{l}$. Given any linear function $\mu$ on $\mathfrak{h}^{\prime}$ we define a representation $\pi_{\mu}^{\prime}$ of $\mathfrak{A}^{\prime}$ on $(\mathfrak{S}$ as follows. $\pi_{\mu}^{\prime}(1)=I$ where $I$ is the identity mapping of (B) and

$$
\begin{aligned}
& \pi_{\mu}^{\prime}\left(Y_{i}^{\prime}\right) \eta_{j_{1}} \eta_{j_{2}} \cdots \eta_{j_{r}}=\eta_{i} \eta_{j_{1}} \eta_{j_{2}} \cdots \eta_{j_{r}}, \\
& \quad \pi_{\mu}^{\prime}\left(H^{\prime}\right) \eta_{j_{1}} \eta_{j_{2}} \cdots \eta_{j_{r}} \\
& \quad=-\left\{\alpha_{j_{1}}\left(H^{\prime}\right)+\alpha_{j_{2}}\left(H^{\prime}\right)+\cdots+\alpha_{j_{r}}\left(H^{\prime}\right)-\mu\left(H^{\prime}\right)\right\}_{\eta_{j_{1}} \eta_{j_{2}} \cdots \eta_{j_{r}}}
\end{aligned}
$$

where $H^{\prime} \in \mathfrak{h}^{\prime}, 1 \leqq i \leqq l, 1 \leqq j_{1}, \cdots, j_{r} \leqq l, r \geqq 0$ and $\eta_{j_{1}} \cdots \eta_{j_{r}}=1$ if $r=0$. Finally $\pi_{\mu}^{\prime}\left(X_{i}^{\prime}\right)$ is defined by induction on $r$ in the following way.

$$
\begin{aligned}
\pi_{\mu}^{\prime}\left(X_{i}^{\prime}\right) 1= & 0, \\
\pi_{\mu}^{\prime}\left(X_{i}^{\prime}\right) \eta_{j_{1}} \cdots \eta_{j_{r}}= & \delta_{i_{1} j_{\mu}} \pi_{\mu}^{\prime}\left(H_{i}^{\prime}\right) \eta_{j_{2}} \cdots \eta_{j_{r}} \\
& +\eta_{j_{1}}\left(\pi_{\mu}^{\prime}\left(X_{i}^{\prime}\right) \eta_{j_{2}} \cdots \eta_{j}\right)
\end{aligned}
$$


where $\delta_{i j}$ is the usual Kronecker symbol. Since $\mathfrak{A}^{\prime}$ is a free algebra and $\eta_{j_{1}} \cdots \eta_{j_{r}}, 1 \leqq j_{1}, \cdots, j_{r} \leqq l, r \geqq 0$, form a base for $\left(\mathcal{S}\right.$ it is clear that $\pi_{\mu}^{\prime}$ is uniquely defined by the above equations. It is easily verified that the kernel of $\pi_{\mu}^{\prime}$ contains the set $\subseteq$ and therefore $\mathfrak{U}^{\prime}$. Now suppose $H^{\prime} \in \mathfrak{h}^{\prime}$ and $\pi_{\mu}^{\prime}\left(H^{\prime}\right)=0$. Then

$$
\alpha_{j_{1}}\left(H^{\prime}\right)+\alpha_{j_{2}}\left(H^{\prime}\right)+\cdots+\alpha_{j_{r}}\left(H^{\prime}\right)-\mu\left(H^{\prime}\right)=0
$$

for all $1 \leqq j_{1}, \cdots, j_{r} \leqq l$. This implies that $\alpha_{j}\left(H^{\prime}\right)=0$ for all $1 \leqq j \leqq l$. Since $\alpha_{j}$ are linearly independent, $H^{\prime}=0$. Hence it is clear that $\mathfrak{h}^{\prime} \cap \mathfrak{u}^{\prime}=\{0\}$.

Let $\mathfrak{A}$ be the factor algebra $\mathfrak{H}^{\prime} / \mathfrak{u}^{\prime}$ and let $X_{\boldsymbol{i}}, Y_{i}, H_{i}$ respectively be the images of $X_{\mathfrak{i}}^{\prime}, Y_{\mathfrak{i}}^{\prime}, H_{i}^{\prime}$ in $\mathfrak{A}, 1 \leqq i \leqq l$. Since $\mathfrak{h}^{\prime} \cap \mathfrak{u}^{\prime}=\{0\}, \mathfrak{h}^{\prime}$ is mapped isomorphically under the natural mapping of $\mathfrak{A}^{\prime}$ on $\mathfrak{A}$, on the linear subspace $\mathfrak{h}$ of $\mathfrak{A}$ spanned by $H_{\boldsymbol{i}}, 1 \leqq i \leqq l$. Hence $\operatorname{dim} \mathfrak{h}=\operatorname{dim} \mathfrak{h}^{\prime}=l$ and every linear function $\lambda$ on $\mathfrak{h}^{\prime}$ can also be regarded as a linear function on $\mathfrak{h}$ if we put $\lambda\left(H_{i}\right)=\lambda\left(H_{i}^{\prime}\right)$, $1 \leqq i \leqq l$. In particular this holds for $\alpha_{j}$ and therefore $\alpha_{j}\left(H_{i}\right)=-a_{j i}$. Since the kernel of $\pi_{\mu}^{\prime}$ contains $\mathfrak{u}^{\prime}, \pi_{\mu}^{\prime}$ defines in the obvious way a representation $\pi_{\mu}$ of $\mathfrak{A}$ on $\mathfrak{B}$.

Consider the representation $\pi^{\prime}$ of $\mathfrak{A}^{\prime}$ on $\mathfrak{A}$ defined as follows:

$$
\begin{array}{rrr}
\pi^{\prime}(1) a=a, & \pi^{\prime}\left(X_{i}^{\prime}\right) a=\left[X_{i}^{\prime}, a\right], & \pi^{\prime}\left(Y_{i}^{\prime}\right) a=\left[Y_{i}, a\right], \\
\pi^{\prime}\left(H_{i}^{\prime}\right) a=\left[H_{i}{ }^{\prime}, a\right], & 1 \leqq i \leqq l, a \in \mathfrak{A} .
\end{array}
$$

It is easily seen that $\pi^{\prime}$ maps every element of the set $\subseteq$ into zero. Hence the kernel of $\pi^{\prime}$ contains $\mathfrak{u}^{\prime}$. Therefore $\pi^{\prime}$ actually determines a representation $\pi$ of $\mathfrak{A}=\mathfrak{H}^{\prime} / \mathfrak{U}^{\prime} . \pi$ is called the adjoint representation of $\mathfrak{A}$ and we shall write ad $z$ instead of $\pi(z)$ for any $z \in \mathfrak{A}$.

The subspace $\mathfrak{h}$ of $\mathfrak{A}$ is an abelian Lie algebra under the bracket operation. Given a representation $\theta$ of this Lie algebra on a vector space $V$ and a linear function $\lambda$ on $\mathfrak{h}$ we denote by $V_{\lambda}$ the subspace of $V$ consisting of all elements $\psi \in V$ such that $\theta(H) \psi=\lambda(H) \psi(H \in \mathfrak{h})$.

Lemma 1. The sum $\sum_{\lambda} V_{\lambda}$, where $\lambda$ runs over all linear functions on $\mathfrak{h}$, is direct. If $U$ is any subspace of $V$ which is invariant under $\theta(\mathfrak{h})$ then

$$
U \cap\left(\sum_{\lambda} V_{\lambda}\right)=\sum_{\lambda}\left(U \cap V_{\lambda}\right) .
$$

Let $\psi \in U \cap\left(\sum_{\lambda} V_{\lambda}\right)$. Then $\psi=\psi_{1}+\cdots+\psi_{r}$ where $\psi_{i} \in V_{\lambda_{i}}$ and $\lambda_{i} \neq \lambda_{j}$, $1 \leqq i, j \leqq r, i \neq j$. We claim that $\psi_{i} \in U, 1 \leqq i \leqq r$. If $r=1$ this is true trivially. Hence we may assume $r>1$ and use induction on $r$. We can find an $H \in \mathfrak{h}$ such that $\lambda_{i}(H) \neq \lambda_{1}(H), 2 \leqq i \leqq r$. Then

$$
\theta(H) \psi=\sum_{1 \leqq i \leqq r} \lambda_{i}(H) \psi_{i} \in U .
$$

Hence 


$$
\theta(H) \psi-\lambda_{1}(H) \psi=\sum_{2 \leqq i \leqq r}\left\{\lambda_{i}(H)-\lambda_{1}(H)\right\} \psi_{i} \in U
$$

Therefore by induction hypothesis $\left\{\lambda_{i}(H)-\lambda_{1}(H)\right\} \psi_{i} \in U, 2 \leqq i \leqq r$. But $\lambda_{i}(H)-\lambda_{1}(H) \neq 0$ for $2 \leqq i \leqq r$. Hence $\psi_{i} \in U, 2 \leqq i \leqq r$ and therefore $\psi_{i} \in U$, $1 \leqq i \leqq r$.

If we take $U=\{0\}$ above it follows that the sum $\sum_{\lambda} V_{\lambda}$ is direct. Also the above proof shows that $U \cap\left(\sum_{\lambda} V_{\lambda}\right)=\sum_{\lambda}\left(V_{\lambda} \cap U\right)$.

Let $\pi$ be a representation of $\mathfrak{A}$ on a vector space $V$. $\lambda$ being any linear function on $\mathfrak{h}$ we define $V_{\lambda}$ as above to be the set of all $\psi \in V$ such that $\pi(H) \psi$ $=\lambda(H) \psi(H \in \mathfrak{h}) . \psi$ is said to be homogeneous of weight $\lambda$ (or to have weight $\lambda$, or to belong to the weight $\lambda$ ) if $\psi \in V_{\lambda}$ and $\lambda$ is called a weight of $\pi$ if $V_{\lambda} \neq\{0\}$. It is clear that if $\psi$ has the weight $\lambda$ and $\lambda$ is not a weight then $\psi=0$. We shall call the dimension of $V_{\lambda}$ the multiplicity of $\lambda$ in $\pi$. Now in particular we may take $\pi$ to be the adjoint representation of $\mathfrak{A}$ and define the subspaces $\mathfrak{A}_{\lambda}$. A weight of the adjoint representation will be called a rank and an element $z$ will be said to be homogeneous of rank $\lambda$ (or to have rank $\lambda$ ) if $z \in \mathfrak{A}_{\lambda}$.

LEMma 2. $\mathfrak{A}=\sum_{\lambda} \mathfrak{H}_{\lambda}$ and every rank is a linear combination of $\alpha_{i}, 1 \leqq i \leqq l$, with integral coefficients.

Let $P, Q, M$ denote any ordered set of integers as follows:

$$
\begin{array}{rlrl}
P & =\left\{i_{1}, i_{2}, \cdots, i_{p}\right\}, & 1 \leqq i_{1}, \cdots, i_{p} \leqq l, p \geqq 0, \\
Q=\left\{j_{1}, j_{2}, \cdots, j_{q}\right\}, & 1 \leqq j_{1}, \cdots, j_{q} \leqq l, q \geqq 0, \\
M=\left\{m_{1}, \cdots, m_{l}\right\}, & m_{i} \geqq 0,1 \leqq i \leqq l,
\end{array}
$$

where $P$ or $Q$ is empty if $p$ or $q$ is zero. Put $|P|=p,|Q|=q$, rank $P=\alpha_{i_{1}}$ $+\alpha_{i_{2}}+\cdots+\alpha_{i_{p}}$, rank $Q=\alpha_{j_{1}}+\alpha_{j_{2}}+\cdots+\alpha_{j_{q}}$, the rank being understood to be zero in case the set is empty. We denote by $\phi$ the empty set and by 0 the set $M$ all of whose elements are zero. Put

$$
z(Q, M, P)=Y_{j_{1}} Y_{j_{2}} \cdots Y_{j_{q}} H_{1}^{m_{1}} \cdot H_{2}^{m_{2}} \cdots H_{l}^{m_{l}^{l}} X_{i_{1}} X_{i_{2}} \cdots X_{i_{p}}
$$

where $H_{i}^{m}=1$ if $m=0$. Making use of the relations

$$
\begin{gathered}
{\left[H_{i}, H_{j}\right]=0, \quad\left[X_{i}, Y_{i}\right]=H_{i}, \quad\left[X_{i}, Y_{j}\right]=0, \quad i \neq j,} \\
{\left[H, X_{i}\right]=\alpha_{i}(H) X_{i}, \quad\left[H, Y_{i}\right]=-\alpha_{i}(H) Y_{i},}
\end{gathered}
$$

$1 \leqq i, j \leqq l, H \in \mathfrak{h}$, which hold in $\mathfrak{A}$ it follows easily that $\mathfrak{A}$ is spanned by the elements $z(Q, M, P)$ taken together for all $Q, M$, and $P$. Clearly $z(Q, M, P)$ has the rank rank $P$-rank $Q$. Let $P$ denote the set of all linear functions on $\mathfrak{h}$ of the form rank $P$-rank $Q$ for all $P$ and $Q$. Then since $z(Q, M, P)$ span $\mathfrak{A}$, $\mathfrak{A} \subset \sum_{\lambda \in \mathrm{P}} \mathfrak{A}_{\lambda}$. Hence $\mathfrak{A}=\sum_{\lambda \in \mathrm{P}} \mathfrak{A}_{\lambda}=\sum_{\lambda} \mathfrak{A}_{\lambda}$. But from Lemma 1 the sum $\sum_{\lambda} \mathfrak{A}_{\lambda}$ is direct. Hence $\mathfrak{A}_{\lambda}=\{0\}$ if $\lambda \in P$. This proves the lemma.

Given $z \in \mathfrak{A}$ and a linear function $\lambda_{0}$ on $\mathfrak{h}$ it follows from Lemmas 1 and 2 
that we can find a unique element $z_{\lambda_{0}} \in \mathfrak{A}_{\lambda_{0}}$ such that $z-z_{\lambda_{0}} \in \sum_{\lambda \neq \lambda_{0}} A_{\lambda} z_{\lambda_{0}}$ will be called the homogeneous component of $z$ of rank $\lambda_{0}$. Clearly $z_{\lambda}=0$ for all linear functions $\lambda$ except a finite number and $z=\sum_{\lambda} z_{\lambda}$.

Exactly as before we call a linear function $\lambda$ on $\mathfrak{h}$ rational if $\lambda\left(H_{\mathfrak{i}}\right) \in K_{0}$ for all $1 \leqq i \leqq l$. We order rational functions lexicographically with respect to the ordered set $\left\{\alpha_{1}, \cdots, \alpha_{l}\right\}$. Let $\mathfrak{S}$ be the subalgebra of $\mathfrak{A}$ generated by $H_{\boldsymbol{i}}, 1 \leqq i \leqq l$, and 1 and let $\mathfrak{B}$ be the left ideal $\sum_{1 \leqq i \leqq l} \mathfrak{A} X_{\boldsymbol{i}}$.

Lemma 3. $\mathfrak{B}$ coincides with the subspace spanned by all elements of the form $\boldsymbol{z}(Q, M, P),|P|>0$. Further $\mathfrak{B} \supset \sum_{\lambda>0} \mathfrak{A}_{\lambda}$ where $\lambda$ runs over all rational functions on $\mathfrak{h}$ which are greater than 0 .

It is clear from the definition of $\mathfrak{B}$ that $z(Q, M, P) \in \mathfrak{P}$ if $|P|>0$. Conversely let $z \in P$. Then $z=\sum_{1 \leq i \leq l} z_{i} X_{i}\left(z_{i} \in \mathfrak{A}\right)$. Since $z_{i}$ is a linear combination of $z\left(Q, M, P^{\prime}\right)$ and since $z\left(Q, M, P^{\prime}\right) X_{i}=z(Q, M, P)$ with $|P|>0$, the first assertion follows.

Let $z \in \mathfrak{A}_{\lambda}$ where $\lambda$ is a rational function greater than 0 . We know that $z$ is a linear combination of $z(Q, M, P)$. Since $z(Q, M, P)$ has rank rank $P-\operatorname{rank}$ $Q$, we may, in view of Lemma 1 , assume that only such elements $z(Q, M, P)$ appear in this linear combination for which rank $P-\operatorname{rank} Q=\lambda$. Since $\lambda>0$ it follows that rank $P>0$ and therefore $|P|>0$. Hence $z \in \mathfrak{B}$.

LEMMA 4. $\mathfrak{B} \cap \mathfrak{S}=\{0\}$.

Let $z \in \mathfrak{B} \cap \mathfrak{S}$. Then $z=\sum_{m_{1}, \ldots, m_{l}} a\left(m_{1}, \cdots, m_{l}\right) H_{1}^{m_{1}} \cdots H_{l}^{m_{l}}$ where $a\left(m_{1}, \cdots, m_{l}\right) \in K$ and the sum is finite. Let $\mu$ be any linear function on $\mathfrak{h}$ and let $\pi_{\mu}$ be the representation of $\mathfrak{A}$ on $\&$ corresponding to (2). Then if $\mu_{i}=\mu\left(H_{i}\right), 1 \leqq i \leqq l$,

$$
\pi_{\mu}(z) 1=\sum_{m_{1}, \cdots, m_{l}} a\left(m_{1}, \cdots, m_{l}\right) \mu_{1}^{m_{1}} \cdots \mu_{l}^{m_{l}} .
$$

But since $z \in \mathfrak{P}$ and $\pi_{\mu}\left(x_{i}\right) 1=0$ it follows that

$$
\sum_{m_{1}, \cdots, m_{l}} a\left(m_{1}, \cdots, m_{l}\right) \mu_{1}^{m_{1}} \cdots \mu_{l}^{m_{l}}=0 .
$$

This is true for every $\mu$ and therefore for every choice of $\mu_{1}, \mu_{2}, \cdots, \mu_{l} \in K$. Since $K$ is an infinite field it follows that all the coefficients $a\left(m_{1}, \cdots, m_{l}\right)$ are zero. Hence $z=0$.

Let $\Lambda$ be any linear function on $\mathfrak{h}$. Put

$$
\mathfrak{Q}_{\Lambda}=\sum_{1 \leqq i \leqq l} \mathfrak{A}\left(H_{i}-\Lambda\left(H_{i}\right)\right), \quad \mathfrak{S}_{\Lambda}=\sum_{1 \leqq i \leqq l} \mathfrak{S}\left(H_{i}-\Lambda\left(H_{i}\right)\right) .
$$

Lemma 5. $\mathfrak{P}+\mathfrak{Q}_{\Delta} \neq \mathfrak{A}$.

It is sufficient to show that $1 \notin \mathfrak{B}+\mathfrak{Q}_{\Lambda}$. Suppose contrary to the assertion 
$1 \in \mathfrak{P}+\mathfrak{Q}_{\Lambda}$. Then we can find $z_{i}, u_{i} \in \mathfrak{A}$ such that

$$
1=\sum_{1 \leqq i \leqq l} z_{i} X_{i}+\sum_{1 \leqq i \leqq l} u_{i}\left(H_{i}-\Lambda\left(H_{i}\right)\right) .
$$

Since elements having different ranks are linearly independent we may assume that $z_{i}$ has rank $-\alpha_{i}$ and $u_{i}$ is of rank zero. Now

$$
1 \equiv \sum_{1 \leqq i \leqq l} u_{i}\left(H_{i}-\Lambda\left(H_{i}\right)\right) \bmod \mathfrak{P}
$$

and since $u_{i}$ is of rank zero, $u_{i}\left(H_{i}-\Lambda\left(H_{i}\right)\right)=\left(H_{i}-\Lambda\left(H_{i}\right)\right) u_{i}$. Further

$$
u_{i}=\sum a_{i}(Q, M, P) z(Q, M, P) \quad\left(a_{i}(Q, M, P) \in K\right)
$$

where the sum is only over such $(Q, M, P)$ for which $\operatorname{rank} Q=\operatorname{rank} P$ since $u_{i}$ is of rank zero and $z(Q, M, P)$ has the rank rank $P$-rank $Q$. Therefore from Lemma 3 ,

$$
u_{i} \equiv \sum a_{i}(\phi, M, \phi) z(\phi, M, \phi) \bmod \mathfrak{B} .
$$

Since $z(\phi, M, \phi) \in \mathfrak{S}$ it follows that

$$
\sum_{1 \leqq i \leqq l} u_{i}\left(H_{i}-\Lambda\left(H_{i}\right)\right)=\sum_{1 \leqq i \leqq l}\left(H_{i}-\Lambda\left(H_{i}\right)\right) u_{i} \in \mathfrak{P}+\mathfrak{S}_{\Lambda} .
$$

Hence $1 \in \mathfrak{P}+\mathfrak{S}_{\Lambda}$. Therefore $1=z+h$ where $z \in \mathfrak{P}, h \in \mathfrak{S}_{\Delta}$. Hence $1-h=z$ $\in \mathfrak{B} \cap \mathfrak{S}_{\mathrm{S}}=\{0\}$ from Lemma 4 . Therefore $1=h \in \mathfrak{S}_{\Lambda}$. Now consider the representation $\pi_{\Delta}$ of $\mathfrak{A}$ on $(B)$ corresponding to the linear function $\Lambda$ on $\mathfrak{h}$. Since $h \in \mathfrak{S}_{\Lambda}$ it follows from the definition of $\pi_{\Lambda}$ that $\pi_{\Lambda}(h) 1=0$. But since $1=h$, $\pi_{\Lambda}(h) \cdot 1=\pi_{\Lambda}(1) \cdot 1=1$. Since $1 \neq 0$ in $\&$ we get a contradiction. The lemma is therefore proved.

Lемма 6. Let $m$ be any integer greater than or equal to 0 . Then

$$
\left[X_{i}, Y_{i}^{m}\right]=m Y_{i}^{m-1}\left(H_{i}-m+1\right), \quad 1 \leqq i \leqq l,
$$

where by definition $Y_{i}^{p}=1$ if $p \leqq 0$.

The assertion is clearly true for $m=0,1$. Hence we may assume $m \geqq 2$ and use induction. Then

$$
\begin{aligned}
{\left[X_{i}, Y_{i}^{m}\right] } & =\left[X_{i}, Y_{i}\right] Y_{i}^{m-1}+Y_{i}\left[X_{i}, Y_{i}^{m-1}\right] \\
& =H_{i} Y_{i}^{m-1}+(m-1) Y_{i}^{m-1}\left(H_{i}-m+2\right)
\end{aligned}
$$

by induction hypothesis. But $\left[H_{i}, Y_{i}\right]=-\alpha_{i}\left(H_{i}\right) Y_{i}=-2 Y_{i}$. Therefore $H_{i} Y_{i}=Y_{i}\left(H_{i}-2\right)$ and

$$
\begin{aligned}
{\left[X_{i}, Y_{i}^{m}\right] } & =Y_{i}^{m-1}\left(H_{i}-2 m+2\right)+(m-1) Y_{i}^{m-1}\left(H_{i}-m+2\right) \\
& =m Y_{i}^{m-1}\left(H_{i}-m+1\right) .
\end{aligned}
$$


Now put $\theta_{i i}=0$ and

$$
\theta_{i j}=\left(\operatorname{ad} Y_{i}^{a_{i j}+1}\right) Y_{j},
$$

$1 \leqq i, j \leqq l$. This is well defined since $a_{j i} \geqq 0$ for $i \neq j$.

Lemma $7\left(^{4}\right) .\left[X_{k}, \theta_{i j}\right]=0$ for all $1 \leqq i, j, k \leqq l$.

We may assume that $i \neq j$. First suppose $k \neq i$. Then $\left[X_{k}, Y_{i}\right]=0$ and therefore $\left[\operatorname{ad} X_{k}\right.$, ad $\left.Y_{i}\right]=0$. Hence

$$
\begin{aligned}
\left(\operatorname{ad} X_{k}\right) \theta_{i j} & =\operatorname{ad} X_{k}\left(\operatorname{ad} Y_{i}\right)^{a_{i i}+1} Y_{j} \\
& =\left(\operatorname{ad} Y_{i}\right)^{a_{j i}+1}\left(\left[X_{k}, Y_{j}\right]\right) .
\end{aligned}
$$

If $k \neq j,\left[X_{k}, Y_{j}\right]=0$ and we get our result. If $k=j$, we get

$$
\begin{aligned}
{\left[X_{k}, \theta_{i j}\right] } & =\left(\operatorname{ad} Y_{i}\right)^{a_{j i}+1} H_{j} \\
& =\left(\operatorname{ad} Y_{i}\right)^{a_{i i}}\left[Y_{i}, H_{i}\right] \\
& =\alpha_{i}\left(H_{j}\right)\left(\operatorname{ad} Y_{i}\right)^{a_{j i} Y_{i} .}
\end{aligned}
$$

If $a_{j i}>0,\left(\operatorname{ad} Y_{i}\right)^{a_{j i}} Y_{i}=0$. On the other hand if $a_{j i}=0$, it follows from assumption (1) of Theorem 1 that $\alpha_{i}\left(H_{j}\right)=-a_{i j}=0$. Hence in either case $\left[X_{k}, \theta_{i j}\right]=0$.

Finally suppose $k=i$. Then $\left[X_{i}, \theta_{i j}\right]=\operatorname{ad}\left(X_{i} Y_{i}^{a_{i i}+1}\right) Y_{j}$. From Lemma 6 , $X_{i} Y_{i}^{a_{i i}+1}=\left(a_{j i}+1\right) Y_{i}^{a_{j i}}\left(H_{i}-a_{j i}\right)$. Hence

$$
\left[X_{i}, \theta_{i j}\right]=\left(a_{j i}+1\right)\left\{\operatorname{ad} Y_{i}^{a_{j i}} \text { ad }\left(H_{i}-a_{j i}\right)\right\} Y_{j} .
$$

But

$$
\left\{\operatorname{ad}\left(H_{i}-a_{j i}\right)\right\} Y_{j}=\left[H_{i}, Y_{j}\right]-a_{j i} Y_{j}=-\alpha_{j}\left(H_{i}\right) Y_{j}-a_{j i} Y_{j}=0 .
$$

Hence $\left[X_{i}, \theta_{i j}\right]=0$ and the lemma is proved.

Let $\lambda_{i}, 1 \leqq i \leqq l$, be any given set of non-negative integers and let $\Lambda_{0}$ be the linear function on $\mathfrak{h}$ defined by $\Lambda_{0}\left(H_{i}\right)=\lambda_{i}$. We consider the left ideal

$$
\mathfrak{B}_{\Lambda_{0}}=\mathfrak{P}+\mathfrak{Q}_{\Lambda_{0}}+\sum_{1 \leqq i, j \leqq l} \mathfrak{A} \theta_{i} \mathfrak{A} \mathfrak{A}+\sum_{1 \leqq i \leqq l} \mathfrak{A} Y_{i}^{\lambda_{i}+1}
$$

in $\mathfrak{A}$, where $\mathfrak{P}$ and $\mathfrak{Q}_{\Lambda_{0}}$ are defined as in Lemma 5 .

Lemma 8. $\mathfrak{B}_{\Lambda_{0}} \neq \mathfrak{A}$.

Suppose the assertion is false. Then $1 \in \mathfrak{B}_{\Lambda_{0}}$. Hence

$$
1 \equiv z+w \bmod \left(\mathfrak{B}+\mathfrak{Q}_{\Lambda_{0}}\right)
$$

where $z \in \sum_{1 \leqq i \leqq l} \mathfrak{A} Y_{i}^{\lambda_{i}+1}$ and $w \in \sum_{1 \leqq i, j \leqq l} \mathfrak{A} \theta_{i j} \mathfrak{A}$. Notice that $\theta_{i j}$ has the rank $-\left(a_{j i}+1\right) \alpha_{i}-\alpha_{j}$. By considering components of different ranks we can

(4) This lemma is due to Chevalley. 
show, as in the proof of Lemma 5, that $z$ can be chosen to be a linear combination of elements of the form $z(Q, M, P) Y_{i}^{\lambda_{i}+1}$ where rank $P$-rank $Q$ $-\left(\lambda_{i}+1\right) \alpha_{i}=0$. Similarly we may assume that $w$ is a linear combination of elements of the form $z(Q, M, P) \theta_{i j} z\left(Q^{\prime}, M^{\prime}, P^{\prime}\right)$ where rank $P+\operatorname{rank} P^{\prime}$ -rank $Q$-rank $Q^{\prime}-\left(a_{j i}+1\right) \alpha_{i}-\alpha_{j}=0 \quad(i \neq j)$. Now consider a term $z(Q, M, P) Y_{i}^{\lambda_{i}+1}$ such that rank $P$-rank $Q=\left(\lambda_{i}+1\right) \alpha_{i}$. If rank $Q>0$, rank $P>\left(\lambda_{i}+1\right) \alpha_{i}$. Hence $z(\phi, M, P) Y_{i}^{\lambda_{i}+1}$ has rank $P-\left(\lambda_{i}+1\right) \alpha_{i}>0$. Therefore by Lemma 3 , it is contained in $\mathfrak{B}$. Since $\mathfrak{P}$ is a left ideal, $z(Q, M, P) Y_{i}^{\lambda_{i}+1}$ $\in \mathfrak{B}$. Hence the term corresponding to $z(Q, M, P) Y_{i}^{\lambda_{i}+1}$ can be dropped from the above congruence. Therefore we may assume that $z$ is a linear combination of elements of the form $z(\phi, M, P) Y_{i}^{\lambda_{i}+1}$ with rank $P=\left(\lambda_{i}+1\right) \alpha_{i}$. But since $\alpha_{j}, 1 \leqq j \leqq l$, are linearly independent rank $P=\left(\lambda_{i}+1\right) \alpha_{i}$ implies that $z(\phi, 0, P)=X_{i}^{\lambda_{i}+1}$. Hence $z$ is a linear combination of elements of the form $H_{1}^{m_{1}} \cdots H_{l}^{m_{l}} X_{i}^{\lambda_{i}+1} Y_{i}^{\lambda_{i}+1}$. But from Lemma 6 ,

$$
\begin{aligned}
X_{i}^{\lambda_{i}+1} Y_{i}^{\lambda_{i}+1} & =X_{i}^{\lambda_{i}}\left[X_{i}, Y_{i}^{\lambda_{i}+1}\right]+X_{i}^{\lambda_{i}} Y_{i}^{\lambda_{i}+1} X_{i} \\
& \equiv\left(\lambda_{i}+1\right) X_{i}^{\lambda_{i}} Y_{i}^{\lambda_{i}}\left(H_{i}-\lambda_{i}\right) \bmod \mathfrak{B} .
\end{aligned}
$$

Since $H_{i}-\lambda_{i} \in \mathfrak{Q}_{\Lambda_{0}}, X_{i}^{\lambda_{i}+1} Y_{i}^{\lambda_{i}+1} \equiv 0 \bmod \left(\mathfrak{P}+\mathfrak{Q}_{\Lambda_{0}}\right)$. This shows that $z$ can be replaced by zero in our congruence. Hence

$$
1 \equiv w \bmod \left(\mathfrak{B}+\mathfrak{Q}_{\Lambda_{0}}\right)
$$

where $w$ is a linear combination of terms of the form $z(Q, M, P) \theta_{i j} z\left(Q^{\prime}, M^{\prime}, P^{\prime}\right)$ with rank $P+\operatorname{rank} P^{\prime}-\operatorname{rank} Q-\operatorname{rank} Q^{\prime}=\left(a_{j_{i}}+1\right) \alpha_{i}+\alpha_{j}(i \neq j)$. Since we are considering a congruence $\bmod \left(\mathfrak{P}+\mathfrak{Q}_{\Lambda_{0}}\right)$ we may clearly assume in addition that $P^{\prime}=\phi$ and $M^{\prime}=0$. Hence we have only terms of the form $z(Q, M, P) \theta_{i j} z\left(Q^{\prime}, 0, \phi\right)$ with $\operatorname{rank} P-\operatorname{rank} Q-\operatorname{rank} Q^{\prime}=\left(a_{j i}+1\right) \alpha_{i}+\alpha_{j}$. From Lemma $7, X_{k}$ commutes with $\theta_{i j}$. Hence

$$
z(Q, M, P) \theta_{i j} z\left(Q^{\prime}, 0, \phi\right)=z(Q, M, \phi) \theta_{i j} z(\phi, 0, P) z\left(Q^{\prime}, 0, \phi\right) .
$$

But rank $P$-rank $Q^{\prime}=\operatorname{rank} Q+\left(a_{j i}+1\right) \alpha_{i}+\alpha_{j}>0(i \neq j)$ and therefore by Lemma $3, z(\phi, 0, P) z\left(Q^{\prime}, 0, \phi\right) \in \mathfrak{B}$. Therefore $w \in \mathfrak{P}$ and we have $1 \in \mathfrak{P}+\mathfrak{Q}_{\Lambda_{0}}$. But by Lemma 5 this is impossible. Hence $\mathfrak{B}_{\Lambda_{0}} \neq \mathfrak{R}$.

As usual we call a left ideal $\mathfrak{N}$ in $\mathfrak{A}$ maximal if $\mathfrak{N} \neq \mathfrak{A}$ and if there exists no left ideal $\mathfrak{N}^{\prime}$ in $\mathfrak{A}$ such that $\mathfrak{N}^{\prime} \supset \mathfrak{N}, \mathfrak{N}^{\prime} \neq \mathfrak{N}$, and $\mathfrak{N}^{\prime} \neq \mathfrak{R}$.

Lemma 9. Let $\Lambda$ be any linear function on $\mathfrak{h}$. Then there exists at most one maximal ideal $\mathfrak{N}$ in $\mathfrak{A}$ such that $\mathfrak{N} \supset \mathfrak{P}+\mathfrak{Q}_{\Lambda}$.

For suppose $\mathfrak{R}_{1}, \mathfrak{R}_{2}$ are two distinct maximal left ideals containing $\mathfrak{B}+\mathfrak{Q}_{\Lambda}$. Then $\mathfrak{N}_{1}+\mathfrak{N}_{2}=\mathfrak{A}$. Hence $1=z_{1}+z_{2}$ where $z_{i} \in \mathfrak{N}_{i}, i=1$, 2. Notice that if $w \in \mathfrak{N}_{1}$, and $H \in \mathfrak{h}$, then

$$
[H, w]=H w-w(H-\Lambda(H))-\Lambda(H) w \in \mathfrak{R}_{1}
$$


since $\mathfrak{R}_{1} \supset \mathfrak{Q}_{\Lambda}$. Therefore $\mathfrak{R}_{1}$ is invariant under ad $\mathfrak{h}$ and from Lemmas 1 and 2, $\mathfrak{N}_{1}=\sum_{\lambda}\left(\mathfrak{R}_{1} \cap \mathfrak{A}_{\lambda}\right)$. Similarly for $\mathfrak{N}_{2}$. Hence if $z_{i, 0}$ is the homogeneous component of $z_{i}$ of rank zero $z_{i, 0} \in \mathfrak{N}_{i}(i=1,2)$ and $1=z_{1,0}+z_{2,0}$. Therefore we may assume that $z_{1}, z_{2}$ are both of rank zero. But then they can be written as linear combinations of $z(Q, M, P)$ with rank $P-\operatorname{rank} Q=0$. If $|P|>0$, $z(Q, M, P) \in \mathfrak{B}$. On the other hand if $P=\phi$ then $Q=\phi$ since rank $P=\operatorname{rank} Q$ and $z(\phi, M, \phi) \equiv c \bmod \mathfrak{Q}_{\Lambda}(c \in K)$ because $H_{i}-\Lambda\left(H_{i}\right) \in \mathfrak{Q}_{\Lambda}, 1 \leqq i \leqq l$. Hence it follows that $z_{i}-c_{i} \in \mathfrak{P}+\mathfrak{Q}_{\Lambda}(i=1,2)$ for some $c_{1}, c_{2} \in K$. Since $z_{1} \in \mathfrak{N}_{1}$ and $\mathfrak{R}_{1} \supset \mathfrak{P}+\mathfrak{Q}_{\Lambda}, c_{1} \in \mathfrak{R}_{1}$. But $\mathfrak{N}_{1}$ is maximal and therefore $1 \in \mathfrak{R}_{1}$. Hence $c_{1}=0$. Similarly $c_{2}=0$. Hence $z_{1}, z_{2} \in \mathfrak{P}+\mathfrak{Q}_{\Lambda}$ and therefore $1 \in \mathfrak{P}+\mathfrak{Q}_{\Lambda}$. But, in view of Lemma 5 , this is false. Thus the lemma is established.

REMARK. Since $\mathfrak{B}+\mathfrak{Q}_{\Lambda} \neq \mathfrak{A}$ it follows from Zorn's lemma that there exists at least one maximal left ideal in $\mathfrak{A}$ containing $\mathfrak{P}+\mathfrak{Q}_{\Lambda}$. The above lemma then shows that it is unique. However we shall not have to invoke Zorn's lemma for our purpose.

LeMma 10. Let $\pi$ be a representation of $\mathfrak{A}$ on $V$. If $\lambda$ and $\mu$ are linear functions on $\mathfrak{h}$ and $z \in \mathfrak{A}_{\lambda}, \psi \in V_{\mu}$ then $\pi(z) \psi \in V_{\lambda+\mu}$.

Let $H \in \mathfrak{h}$. Then

$$
\begin{aligned}
\pi(H) \pi(z) \psi & =\pi([H, z]) \psi+\pi(z) \pi(H) \psi \\
& =\lambda(H) \pi(z) \psi+\mu(H) \pi(z) \psi \\
& =(\lambda(H)+\mu(H)) \pi(z) \psi .
\end{aligned}
$$

Hence $\pi(z) \psi \notin V_{\lambda+\mu}$.

We now define a linear transformation $\sigma_{i}, 1 \leqq i \leqq l$, in the space of all linear functions on $\mathfrak{h}$ as follows:

$$
\sigma_{i} \lambda=\lambda-\lambda\left(H_{i}\right) \alpha_{i} .
$$

Since $\sigma_{i}^{2}$ is the identity, $\sigma_{i}, 1 \leqq i \leqq l$, generate a group $W$. Further, we recall that a linear function $\lambda$ is called integral if $\lambda\left(H_{i}\right), 1 \leqq i \leqq l$, are all integers.

Lemma 11. Let $\pi$ be a representation of $\mathfrak{A}$ on $V$. Suppose for every $\psi \in V$ we can find an integer $\nu \geqq 0$ such that $\pi\left(X_{i}^{\nu}\right) \psi=\pi\left(Y_{i}^{\nu}\right) \psi=0,1 \leqq i \leqq l$. Then every weight of $\pi$ is an integral function. Also if $\Lambda$ is a weight of $\pi$ then for any $i$, $1 \leqq i \leqq l, \Lambda-k \alpha_{i}$ is also a weight of $\pi$ for every integer $k$ such that

$$
\min \left(0, \Lambda\left(H_{i}\right)\right) \leqq k \leqq \max \left(0, \Lambda\left(H_{i}\right)\right) .
$$

In particular $\sigma \Lambda$ is a weight of $\pi$ for every $\sigma \in W$.

Let $\Lambda$ be any weight of $\pi$. Consider any fixed $i$ and choose an element $\psi \in V_{\Lambda}, \psi \neq 0$. Let $k_{0}$ be the least integer greater than or equal to 0 such that $\pi\left(X_{i}^{k_{1}+1}\right) \psi=0$. Put $\psi_{0}=\pi\left(X_{i}^{k_{0}}\right) \psi \neq 0$. Define $\psi_{k}=\pi\left(Y_{i}^{k}\right) \psi_{0}, k \geqq 1$. Then by hypothesis $\psi_{\nu}=0$ for some integer $\nu \geqq 0$. Let $J$ be the least integer greater than 
or equal to 0 such that $\psi_{J_{+1}}=0$. Put $\psi_{-1}=1$. From Lemma 10 it is clear that $\psi_{k} \in V_{\Delta+\left(k_{0}-k\right)} \alpha_{i}$. Using this fact together with the relation $\left[X_{i}, Y_{i}\right]=H_{i}$ we easily prove by induction on $k$ that

$$
\pi\left(X_{i}\right) \psi_{k}=k\left[\Lambda\left(H_{i}\right)+\left(2 k_{0}-k+1\right)\right] \psi_{k-1}, \quad k \geqq 0 .
$$

On substituting $k=J+1$ and remembering that $\psi_{J+1}=0, \psi_{J} \neq 0, J+1 \neq 0$ we get

$$
J=\Lambda\left(H_{i}\right)+2 k_{0} .
$$

This shows that $\Lambda\left(H_{i}\right)$ is an integer. This being true for every $i, 1 \leqq i \leqq l$, it follows that $\Lambda$ is integral. Further $\psi_{k} \in V_{\Lambda+\left(k_{0}-k\right) \alpha_{i}}$ and $\psi_{k} \neq 0$ for $0 \leqq k \leqq J$. Hence $\Lambda-k \alpha_{i}$ is a weight of $\pi$ for

$$
-k_{0} \leqq k \leqq J-k_{0}=\Lambda\left(H_{i}\right)+k_{0} .
$$

Similarly let $k_{0}^{\prime}$ be the least integer greater than or equal to 0 such that $\pi\left(Y_{i}^{k_{0}^{\prime}+1}\right) \psi=0$. Put $\phi_{0}=\pi\left(Y_{i}^{k_{0}^{\prime}}\right) \psi \neq 0$ and $\phi_{k}=\pi\left(X_{i}^{k}\right) \phi_{0}, k \geqq 1$, and $\phi_{-1}=0$. Again we prove by induction on $k$ that

$$
\pi\left(Y_{i}\right) \phi_{k}=k\left\{-\Lambda\left(H_{i}\right)+\left(2 k_{0}^{\prime}-k+1\right)\right\}_{\phi_{k-1}}, \quad k \geqq 0 .
$$

Let $J^{\prime}$ be the least integer greater than or equal to 0 such that $\phi_{J^{\prime}+1}=0$. Substituting $k=J^{\prime}$ in the above equation we get, as before,

$$
J^{\prime}=-\Lambda\left(H_{i}\right)+2 k_{0}^{\prime} \text {. }
$$

Now $\phi_{k} \in V_{\Lambda}-\left(k_{0}^{\prime}-k\right) \alpha_{i}$ and $\phi_{k} \neq 0$ for $0 \leqq k \leqq J^{\prime}$. Hence $\Lambda-k \alpha_{i}$ is a weight of $\pi$ for

$$
k_{0}^{\prime}-J^{\prime}=-k_{0}^{\prime}+\Lambda\left(H_{i}\right) \leqq k \leqq k_{0}^{\prime} .
$$

Combining this with the earlier result we find that $\Lambda-k \alpha_{i}$ is a weight of $\pi$ for all integers $k$ such that

$$
\min \left\{-k_{0},-k_{0}^{\prime}+\Lambda\left(H_{i}\right)\right\} \leqq k \leqq \max \left\{k_{0}^{\prime}, \Lambda\left(H_{i}\right)+k_{0}\right\} .
$$

Since the integer $k=\Lambda\left(H_{i}\right)$ always lies in this range, $\sigma_{i} \Lambda=\Lambda-\Lambda\left(H_{i}\right) \alpha_{i}$ is a weight. This being true for any $i$ and any weight $\Lambda$ of $\pi$ it follows immediately that $\sigma \Lambda$ is a weight for every $\sigma \in W$. Finally since $k_{0} \geqq 0, k_{0}^{\prime} \geqq 0$,

$$
\begin{aligned}
\min \left\{-k_{0},-k_{0}{ }^{\prime}+\Lambda\left(H_{i}\right)\right\} & \leqq \min \left(0, \Lambda\left(H_{i}\right)\right) \leqq \max \left(0, \Lambda\left(H_{i}\right)\right) \\
& \leqq \max \left\{k_{0}^{\prime}, \Lambda\left(H_{i}\right)+k_{0}\right\} .
\end{aligned}
$$

Therefore the lemma is proved.

We now return to the left ideal $\mathfrak{B}_{\Lambda_{0}}$ of Lemma 8 .

Lemma 12. Let $\pi$ be the natural representation of $\mathfrak{A}$ on $\mathfrak{A} / \mathfrak{B}_{\Lambda_{0}}=\mathfrak{A} *$. Then $\Lambda_{0}$ is a weight of $\pi$, and every weight of $\pi$ is of the form $\Lambda=\Lambda_{0}-\left(d_{1} \alpha_{1}+\cdots+d_{l} \alpha_{l}\right)$ where $d_{i}$ are integers greater than or equal to 0 . Further $\mathfrak{A}^{*}=\sum_{\Lambda} \mathfrak{I}_{\Lambda}^{*}$ where the 
sum is direct and is over all weights $\Lambda$ of $\pi$. Finally, given any $z^{*} \in \mathfrak{Y}^{*}$ we can find an integer $\nu \geqq 0$ such that

$$
\pi\left(X_{i}^{\nu}\right) z^{*}=\pi\left(Y_{i}^{\nu}\right) z^{*}=0
$$

Let $z \rightarrow z^{*}$ denote the natural mapping of $\mathfrak{A}$ on $\mathfrak{A}^{*}$. Since from Lemma 8 , $1 \in \mathfrak{B}_{\Lambda_{0}}, 1^{*} \neq 0$. Also $\mathfrak{B}_{\Lambda_{0}} \supset \mathfrak{Q}_{\Lambda_{0}}$ and therefore it is clear that $1^{*} \in \mathfrak{Q}_{\Lambda_{0}}^{*}$. Hence $\Lambda_{0}$ is a weight of $\pi$.

Given any $z \in \mathfrak{A}$ we can write it as a linear combination of $z(Q, M, P)$. But clearly $z(Q, M, P) \in \mathfrak{P}$ if $|P|>0$ or is congruent to $c z(Q, 0, \phi) \bmod$ $\mathfrak{Q}_{\Lambda_{0}}(c \in K)$ if $|P|=0$. Hence $z$ is congruent mod $\mathfrak{P}+\mathfrak{Q}_{\Lambda_{0}}$ to a linear combination of $z(Q, 0, \phi)$. Therefore $(z(Q, 0, \phi))^{*}$ taken together for all $Q$ span $\mathfrak{A}^{*}$. Since $z(Q, 0, \phi)$ has the rank-rank $Q$ and $(z(Q, 0, \phi))^{*}=\pi(z(Q, 0, \phi)) 1^{*}$ it follows from Lemma 10 that $(z(Q, 0, \phi))^{*} \in \mathfrak{Y}_{\Lambda_{0}-\text { rank } Q \text {. Hence }}^{*}$

$$
\mathfrak{A}^{*}=\sum_{Q} \mathfrak{A}_{\Lambda_{0}-\mathrm{rank} Q}^{*}
$$

We now deduce from Lemma 1 that this sum is direct and every weight $\Lambda$ of $\pi$ is of the form $\Lambda_{0}-\operatorname{rank} Q$. The first part of the lemma is therefore proved.

Since $(z(Q, 0, \phi))^{*}$ span $\mathfrak{A}^{*}$ we may, in proving the second part, assume that $z^{*}=(z(Q, 0, \phi))^{*}$. Hence $z^{*} \in \mathfrak{U}_{\Lambda_{0}-\text { rank }}^{*}$. For a fixed $i$ consider $\pi\left(X_{i}^{\nu}\right) z^{*}$. From Lemma $10, \pi\left(X_{i}^{\nu}\right) z^{*} \in \mathfrak{H}_{\Lambda_{0}-\mathbf{r a n k}}^{*} Q+\nu \alpha_{i}$. Now suppose $\pi\left(X_{i}^{\nu}\right) z^{*} \neq 0$. Then $\Lambda_{0}$-rank $Q+\nu \alpha_{i}$ is a weight and therefore is of the form $\Lambda_{0}$-rank $Q^{\prime}$ for some $Q^{\prime}$. Therefore rank $Q-\nu \alpha_{i}=\operatorname{rank} Q^{\prime}$. Let

$$
\begin{aligned}
& \operatorname{rank} Q=d_{1} \alpha_{1}+\cdots+d_{l} \alpha_{l}, \\
& \operatorname{rank} Q^{\prime}=d_{1}^{\prime} \alpha_{1}+\cdots+d_{l}^{\prime} \alpha_{l} .
\end{aligned}
$$

Then $d_{j}, d_{j}^{\prime}, 1 \leqq j \leqq l$, are all integers greater than or equal to 0 . Since $\alpha_{1}, \cdots, \alpha_{l}$ are linearly independent it follows that $d_{i}-\nu=d_{i}^{\prime}$. Hence $\nu=d_{i}$ $-d_{i}^{\prime} \leqq d_{i}$. Therefore if $\nu>d_{i}, \pi\left(X_{i}^{\nu}\right) z^{*}=0$.

Now we consider $\pi\left(Y_{i}^{\nu}\right) z^{*}$. Let $R_{i}, L_{i}$, and $D_{i}$ denote the linear mappings of $\mathfrak{A}$ defined as follows:

$$
R_{i} w=w Y_{i}, \quad L_{i} w=Y_{i} w, \quad D_{i} w=\left[Y_{i}, w\right] \quad(w \in \mathfrak{A}) .
$$

Clearly $L_{i}=D_{i}+R_{i}$ and $L_{i}, R_{i}, D_{i}$ all commute with each other. Hence for any integer $m \geqq 0$

$$
L_{i}^{m}=\left(D_{i}+R_{i}\right)^{m}=\sum_{0 \leqq p \leqq m} \frac{m !}{m-p ! p !} R_{i}^{m-p} D_{i}^{p}
$$

(where $L_{i}^{p}=R_{i}^{p}=D_{i}^{p}$ is the identity mapping if $p=0$ ). Notice that $D_{i}^{a{ }^{+1}{ }^{+1}} Y_{j}=\theta_{i j}$ if $i \neq j$ and $D_{i} Y_{i}=0$. Hence we can find an integer $\nu_{0}$ such that if $p>\nu_{0}$, $D_{i}^{p} Y_{j} \in \mathfrak{B}, 1 \leqq j \leqq l$, where $\mathfrak{B}$ is the ideal $\sum_{1 \leqq j, k \leqq l} \mathfrak{A} \theta_{j k} \mathfrak{A}$. We claim that if 
$p>\nu_{0}|Q|, D_{i}^{p} z(Q, 0, \phi) \in \mathfrak{B}$. This is easily proved by induction on $|Q|$ if we make use of the rule

$$
D_{i}^{p}(u v)=\sum_{0 \leqq r \leqq p} \frac{p !}{p-r ! r !} D_{i}^{r}(u) D_{i}^{p-r}(v) \quad(u, v \in \mathfrak{U}) .
$$

Let $\nu=\nu_{0}|Q|+\max _{1 \leqq j \leqq l} \lambda_{j}$ where $\lambda_{j}=\Lambda_{0}\left(H_{j}\right)$ and let $m>\nu$. Then from (6),

$$
Y_{i z}^{m} z(Q, 0, \phi)=\sum_{0 \leqq p \leqq m} \frac{m !}{m-p ! p !}\left\{D_{i}^{p}(z(Q, 0, \phi)\} Y_{i}^{m-p} .\right.
$$

Now if $p>\nu_{0}|Q|, D_{i}^{p}(z(Q, 0, \phi)) \in \mathfrak{B}$ and if $p \leqq \nu_{0}|Q|, m-p \geqq \lambda_{i}+1$ and

$$
\left\{D_{i}^{p}(z(Q, 0, \phi))\right\} Y_{i}^{m-p} \in \mathfrak{A} Y_{i}^{\lambda_{i}+1} \subset \mathfrak{B}_{\Lambda_{0}} .
$$

Therefore $Y_{i}^{m} z(Q, 0, \phi) \in \mathfrak{B}_{\Lambda_{0}}$ and

$$
\pi\left(Y_{i}^{m}\right)(z(Q, 0, \phi))^{*}=0 .
$$

Thus the lemma is proved.

Let $\pi$ be as above. We shall call a weight $\Lambda$ of $\pi$ extreme if it is impossible to find an $\alpha_{i}, 1 \leqq i \leqq l$, and $\sigma \in W$ such that $\Lambda+\sigma \alpha_{i}$ and $\Lambda-\sigma \alpha_{i}$ are both weights of $\pi$. Obviously $\Lambda_{0}$ is the highest weight of $\pi$ and therefore it is also extreme. From Lemmas 11 and 12 it is clear that if $\Lambda$ is a weight of $\pi$ then $\sigma \Lambda$ is also a weight of $\pi$ for all $\sigma \in W$.

LemMA 13. Let $\pi$ be as in Lemma 12 and let $\Lambda$ be an extreme weight of $\pi$. Then for any $\sigma \in W, \sigma \Lambda$ is also an extreme weight of $\pi$.

Suppose $\sigma \Lambda$ is not extreme. Then for some $\alpha_{i}$ and $\sigma_{0} \in W, \sigma \Lambda+\sigma_{0} \alpha_{i}$ and $\sigma \Lambda-\sigma_{0} \alpha_{i}$ are both weights of $\pi$. But it follows from Lemmas 11 and 12 that $\sigma^{-1}$ $\left(\sigma \Lambda+\sigma_{0} \alpha_{i}\right)=\Lambda+\sigma^{-1} \sigma_{0} \alpha_{i}$ and $\sigma^{-1}\left(\sigma \Lambda-\sigma_{0} \alpha_{i}\right)=\Lambda-\sigma^{-1} \sigma_{0} \alpha_{i}$ are also weights of $\pi$. Since $\sigma^{-1} \sigma_{0} \in W$ this contradicts the hypothesis that $\Lambda$ is extreme. Hence $\sigma \Lambda$ must be extreme.

LEMMA 14. Let $\pi$ be as above and let $\Lambda$ be an extreme weight of $\pi$ such that $\sigma_{i} \Lambda \geqq \Lambda$ for all $1 \leqq i \leqq l$. Then $\Lambda-\alpha_{i}$ is not a weight for any $\sigma_{i}, 1 \leqq i \leqq l$.

Suppose $\Lambda-\alpha_{i}$ is a weight. Since $\sigma_{i} \Lambda=\Lambda-\Lambda\left(H_{i}\right) \alpha_{i} \geqq \Lambda, \Lambda\left(H_{i}\right) \leqq 0$. From Lemma $11, \Lambda-\nu \alpha_{i}$ is a weight for $\Lambda\left(H_{i}\right) \leqq \nu \leqq 0$. Hence if $\Lambda\left(H_{i}\right)<0, \Lambda+\alpha_{i}$ is a weight. On the other hand if $\Lambda\left(H_{i}\right)=0, \sigma_{i}\left(\Lambda-\alpha_{i}\right)=\Lambda+\alpha_{i}$ is again a weight. Therefore in either case both $\Lambda+\alpha_{i}$ and $\Lambda-\alpha_{i}$ are weights, thus contradicting our hypothesis that $\Lambda$ is extreme.

Notice that $\sigma_{i} \alpha_{j}=\alpha_{j}-\alpha_{j}\left(H_{i}\right) \alpha_{i}=\alpha_{j}+a_{j i} \alpha_{i}$. Since $\alpha_{i}, 1 \leqq i \leqq l$, form a base for the space of all linear functions on $\mathfrak{h}$ it follows that the group $W$ generated by $\sigma_{i}$ is exactly the same as that appearing in the statement of Theorem 1. So far we have made no use of the hypothesis that $W$ is a finite group. But now it will enter in an essential way in the proof. 
LEMmA 15. If $W$ is a finite group the space $\mathfrak{A}^{*}=\mathfrak{A} / \mathfrak{B}_{\Lambda_{0}}$ is finite-dimensional.

Let $\pi$ be the natural representation of $\mathfrak{A}$ on $\mathfrak{A}^{*}$ and let $\subseteq$ denote the set of all weights of $\pi$ of the form $\sigma \Lambda_{0}(\sigma \in W)$. Since $W$ is finite, $\subseteq$ is a finite set. Also since $\Lambda_{0}$ is extreme it follows from Lemma 13 that every weight in $\mathfrak{S}$ is extreme. Let $\Lambda_{1}$ be the lowest weight in $\mathfrak{S}$. Then $\sigma_{i} \Lambda_{1} \geqq \Lambda_{1}$ for all $1 \leqq i \leqq l$ and $\Lambda_{1}$ is an extreme weight. Hence from Lemma $14, \Lambda_{1}-\alpha_{i}$ is not a weight of $\pi$ for any $\alpha_{i}, 1 \leqq i \leqq l$. Choose any $z^{*} \in \mathfrak{A}_{\Lambda_{1}}^{*}\left(z^{*} \neq 0\right)$ and put $\mathfrak{N}^{*}=\pi(\mathfrak{U}) z^{*}$. Then $\mathfrak{N}^{*}$ is an invariant subspace of $\mathfrak{A}^{*}$. We claim that $\mathfrak{N}^{*}$ is finite-dimensional. Corresponding to any $P, M, Q$ put

$$
w(P, M, Q)=X_{i_{1}} X_{i_{2}} \cdots X_{i_{p}} H_{1}^{m_{i}} \cdots H_{l}^{m_{l}} Y_{j_{1}} Y_{j_{2}} \cdots Y_{j_{q}}
$$

where $P=\left\{i_{1}, \cdots, i_{p}\right\}, M=\left\{m_{1}, \cdots, m_{l}\right\}$, and $Q=\left\{j_{1}, \cdots, j_{q}\right\}$ as in (3). Then exactly as in the case of $z(Q, M, P)$ we prove that $w(P, M, Q)$ taken together for all $P, M, Q$ span $\mathfrak{A}$. From Lemma 10, $\pi\left(Y_{i}\right) z^{*} \in \mathfrak{A}_{\Lambda_{1}-\alpha_{i}}^{*}$. But since $\Lambda_{1}-\alpha_{i}$ is not a weight of $\pi, \pi\left(Y_{i}\right) z^{*}=0$. Hence $\pi(w(P, M, Q)) z^{*}$ $=0$ if $|Q|>0$. Also $\pi(H) z^{*}=\Lambda_{1}(H) z^{*}(H \in \mathfrak{h})$. Hence it is obvious that $\mathfrak{R}^{*}$ is spanned by elements of the form $\pi(w(P, 0, \phi)) z^{*}$. But again by Lemma 10 ,

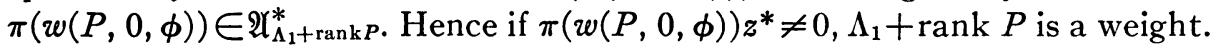
Therefore from Lemma 12,

$$
\Lambda_{1}+\operatorname{rank} P=\Lambda_{0}-\operatorname{rank} Q
$$

for some $Q$. Also $\Lambda_{1}=\Lambda_{0}-\operatorname{rank} Q^{\prime}$ for a suitable $Q^{\prime}$. Therefore

$$
\text { rank } P=\operatorname{rank} Q^{\prime}-\operatorname{rank} Q \text {. }
$$

Let rank $P=e_{1} \alpha_{1}+\cdots+e_{l} \alpha_{l}$, rank $Q=d_{1} \alpha_{1}+\cdots+d_{l} \alpha_{l}$, rank $Q^{\prime}=d_{1}^{\prime} \alpha_{1}$ $+\cdots+d_{l}^{\prime} \alpha_{l}$. Then $e_{i}, d_{i}, d_{i}^{\prime} \geqq 0,1 \leqq i \leqq l$, and again from the linear independence of $\alpha_{i}$ we deduce that $e_{i}=d_{i}^{\prime}-d_{i}, 1 \leqq i \leqq l$. Hence $e_{i} \leqq d_{i}^{\prime}$. Therefore $|P|=\sum e_{i} \leqq \sum d_{i}^{\prime}=\left|Q^{\prime}\right|$. Thus it is clear that there are only a finite number of possibilities for $P$. Since $\mathfrak{N}^{*}$ is spanned by $\pi(w(P, 0, \phi)) z^{*}$ with $|P| \leqq\left|Q^{\prime}\right|$, it follows that $\operatorname{dim} \mathfrak{R}^{*}$ is finite. Also since $z^{*} \in \mathfrak{R}^{*}, z^{*} \neq 0, \mathfrak{R}^{*} \neq\{0\}$.

Since $\mathfrak{N}^{*}$ is invariant under $\pi$, it follows from Lemmas 1 and 12 that $\mathfrak{N}^{*}=\sum_{\Lambda} \mathfrak{N}_{\Lambda}^{*}$ where $\mathfrak{N}_{\Lambda}^{*}=\mathfrak{A}_{\Delta}^{*} \cap \mathfrak{N}^{*}$ and $\Lambda$ runs over all the weights of $\pi$. Since the sum $\sum_{\Lambda} \mathfrak{A}_{\Lambda}^{*}$ is direct and $\operatorname{dim} \mathfrak{N}^{*}$ is finite, $\mathfrak{N}_{\Lambda}^{*} \neq\{0\}$ for only a finite number of weights $\Lambda$. Let $\pi^{*}$ denote the representation of $\mathfrak{A}$ induced on $\mathfrak{N}^{*}$. Then it is obvious from the above remark that $\pi^{*}$ has only a finite number of weights. Let $\Lambda$ be a weight of $\pi^{*}$ and let $w^{*} \in \mathfrak{N}_{\Lambda}^{*}$. Then by Lemma 10, $\pi^{*}\left(X_{i}^{\nu}\right) w^{*} \in \mathfrak{N}_{\Lambda+\nu \alpha_{i}}^{*}$. Since $\alpha_{i} \neq 0$, the linear functions $\Lambda+\nu \alpha_{i}, \nu=1,2, \cdots$, are all distinct. Hence they cannot all be weights of $\pi^{*}$. Hence for sufficiently large $\nu, \pi^{*}\left(X_{i}^{\nu}\right) w^{*}=0$. Similarly we prove that $\pi^{*}\left(Y_{i}^{\nu}\right) w^{*}=0$ for $\nu$ sufficiently large. Since $\mathfrak{N}^{*}=\sum_{\Lambda} \mathfrak{N}_{\Lambda}^{*}$ it follows that for any $w^{*} \in \mathfrak{N}^{*}$ we can find an integer $\nu \geqq 0$ such that $\pi^{*}\left(X_{i}^{\nu}\right) w^{*}=\pi^{*}\left(Y_{i}^{\nu}\right) w^{*}=0,1 \leqq i \leqq l$. Hence Lemma 11 is applicable. Since $z^{*} \in \mathfrak{N}_{\Lambda_{1}}^{*}\left(z^{*} \neq 0\right)$ it follows that $\Lambda_{1}$ is a weight of $\pi^{*}$. But 
$\Lambda_{1} \in \Im$ and therefore $\Lambda_{1}=\sigma \Lambda_{0}(\sigma \in W)$. Therefore from Lemma $11, \sigma^{-1} \Lambda_{1}=\Lambda_{0}$ is also a weight of $\pi^{*}$. Now $(z(Q, 0, \phi))^{*}$ has the weight $\Lambda_{0}-\operatorname{rank} Q$. Hence $(z(Q, 0, \phi))^{*} \in \mathfrak{A}_{\Lambda_{0}}^{*}$ and $(z(Q, 0, \phi))^{*} \neq 0$ implies that rank $Q=0$, that is, $Q=\phi$. Since the elements $(z(Q, 0, \phi))^{*} \operatorname{span} \mathfrak{A}^{*}$, it follows from Lemma 1 that $\mathfrak{A}_{\Lambda_{0}}^{*}=K \cdot 1^{*}$. Since $\mathfrak{N}_{\Lambda_{0}}^{*} \neq\{0\}$ it follows that $1^{*} \in \mathfrak{N}^{*}$. Hence

$$
\mathfrak{U}^{*}=\pi(\mathfrak{U}) 1^{*} \subset \mathfrak{N}^{*} \text {. }
$$

Therefore $\mathfrak{I}^{*}$ is finite-dimensional.

Let $\mathfrak{M}^{*}$ be an invariant subspace of $\mathfrak{U}^{*}$ of the maximum possible dimension such that $\mathfrak{M}^{*} \neq \mathfrak{N}^{*}$. Let $\mathfrak{M}_{\Lambda_{0}}$ be the complete inverse image of $\mathfrak{M}^{*}$ in $\mathfrak{A}$. Clearly $\mathfrak{M}_{\Lambda_{0}}$ is a maximal left ideal containing $\mathfrak{B}_{\Lambda_{0}}$. Since $\mathfrak{B}_{\Lambda_{0}} \supset \mathfrak{P}+\mathfrak{Q}_{\Lambda_{0}}$, it follows from Lemma 9 that $\mathfrak{M}_{\Lambda_{0}}$ is the unique maximal left ideal containing $\mathfrak{B}_{\Lambda_{0}}$. The natural representation $\pi_{\Lambda_{0}}$ on $\mathfrak{A} / \mathfrak{M}_{\Lambda_{0}} \cong \mathfrak{H}^{*} / \mathfrak{M}^{*}$ is then irreducible and finite-dimensional. We note for later use that if $\Lambda_{0}=0$ then $\pi_{\Lambda_{0}}\left(X_{i}\right)=\pi_{\Lambda_{0}}\left(Y_{i}\right)$ $=\pi_{\Lambda_{0}}\left(H_{i}\right)=0,1 \leqq i \leqq l$. This follows from the fact that $X_{i}, Y_{i}, H_{i} \in \mathfrak{B}_{\Delta_{0}}$ and therefore $\mathfrak{A} / \mathfrak{B}_{\Lambda_{0}}=K \cdot 1^{*}$.

Let $\pi$ be any representation of $\mathfrak{A}$ on a vector space $V$. We shall say that $\lambda$ is the highest weight of $\pi$ if $\lambda$ is a weight of $\pi$ and for any weight $\mu$ of $\pi(\mu \neq \lambda)$, $\lambda-\mu$ is a rational function greater than 0 . Given any linear function $\Lambda$ on $\mathfrak{h}$, let $\mathfrak{M}_{\Lambda}$ be the unique maximal ideal containing $\left(^{5}\right) \mathfrak{B}+\mathfrak{Q}_{\Lambda}$. We denote by $\pi_{\Lambda}$ the natural representation of $\mathfrak{A}$ on $\mathfrak{A} / \mathfrak{M}_{\Lambda}$. Since $\mathfrak{M}_{\Lambda}$ is maximal, $\pi_{\Lambda}$ is irreducible. It is easily seen that $\Lambda$ is the highest weight of $\pi_{\Lambda}$.

LEMMA 16. Let $\pi$ be an irreducible representation of $\mathfrak{A}$ on $V$ such that $\Lambda$ is the highest weight of $\pi$. Then $\pi$ is equivalent $\left({ }^{6}\right)$ to $\pi_{\Delta}$. Also $\operatorname{dim} V_{\Delta}=1$.

Let $\psi \in V_{\Lambda}, \psi \neq 0$. Since $\pi$ is irreducible, $\pi(\mathfrak{A}) \psi=V$. Let $\mathfrak{M}$ be the left ideal in $\mathfrak{A}$ consisting of all elements $z$ such that $\pi(z) \psi=0$. Put $\mathfrak{A} *=\mathfrak{A} / \mathfrak{M}$ and let $z \rightarrow z^{*}$ denote the natural mapping of $\mathfrak{A}$ on $\mathfrak{U}^{*}$. Let $\theta$ be the linear mapping of $\mathfrak{A}^{*}$ into $V$ defined as follows. For any $z \in \mathfrak{U}$ put $\theta z^{*}=\pi(z) \psi$. It is easily seen that this mapping is well defined. Since $V=\pi(\mathfrak{A}) \psi, \theta$ maps $\mathfrak{A}^{*}$ onto $V$. If $\theta z^{*}=0$ then $\pi(z) \psi=0$. Hence $z \in \mathfrak{M}$ and so $z^{*}=0$. Therefore $\theta$ is an isomorphism of $\mathfrak{A}^{*}$ on $V$. Let $\pi^{*}$ be the natural representation of $\mathfrak{A}$ on $\mathfrak{A}^{*}$. Then if $w, z \in \mathfrak{A}$,

$$
\begin{aligned}
\theta \pi^{*}(w) z^{*} & =\theta(w z)^{*}=\pi(w z) \psi=\pi(w) \pi(z) \psi \\
& =\pi(w) \theta z^{*} .
\end{aligned}
$$

This shows that $\pi$ and $\pi^{*}$ are equivalent. Since $\pi$ is irreducible the same

(5) We have assumed the existence of $\mathfrak{M}_{\Lambda}$ here and therefore made use of Zorn's lemma. This is done only for convenience. It would be sufficient for our purpose to define $\pi_{\Lambda}$ whenever $\mathfrak{M}_{\mathbf{\Lambda}}$ exists.

(6) $\mathfrak{A}$ being any associative algebra and $\pi, \pi^{\prime}$ two representations of $\mathfrak{A}$ on $V, V^{\prime}$ respectively we say that $\pi$ and $\pi^{\prime}$ are equivalent if there exists an isomorphism $\theta$ of $V$ onto $V^{\prime}$ such that $\pi^{\prime}(z) \theta \psi=\theta \pi(z) \psi$ for every $\psi \in V$ and $z \in \mathscr{R}$. 
holds for $\pi^{*}$ and therefore $\mathfrak{M}$ is a maximal left ideal. Now it follows from Lemma 10 that

$$
\pi\left(X_{i}\right) \psi \in V_{\Lambda+\alpha_{i}} .
$$

Since $\Lambda$ is the highest weight of $\pi, \Lambda+\alpha_{i}$ is not a weight of $\pi$. Hence $\pi\left(X_{i}\right) \psi=0$. Also since $\psi \in V_{\Delta}$,

$$
\{\pi(H)-\Lambda(H) \dot{\xi} \psi=0
$$

Since $\mathfrak{M}$ is a left ideal these relations imply that $\mathfrak{M} \supset \mathfrak{P}+\mathfrak{Q}_{\Lambda}$. Since $\mathfrak{M}$ is maximal it must coincide with $\mathfrak{M}_{\Lambda}$. Hence $\pi^{*}=\pi_{\Lambda}$. Finally, since $\mathfrak{M} \supset \mathfrak{P}+\mathfrak{Q}_{\Lambda}$ it is clear that $(z(Q, 0, \phi))^{*}$ span $\mathfrak{A}^{*}$. Since $z(Q, 0, \phi)$ is of rank rank $Q$, $(z(Q, 0, \phi))^{*} \in \mathfrak{H}_{\Lambda-\text { rank } \boldsymbol{Q}}^{*}$. Therefore $\mathfrak{A}_{\Lambda}^{*}$ is spanned by $1^{*}$. Since $1 \notin \mathfrak{M}$, dim $\mathfrak{A}_{\Lambda}^{*}$ $=1$. Since $\pi$ and $\pi^{*}$ are equivalent $\operatorname{dim} V_{\Delta}=\operatorname{dim} \mathfrak{R}_{\Lambda}^{*}=1$. The lemma is therefore proved.

We recall that an integral function $\Lambda$ on $\mathfrak{h}$ is called dominant if $\Lambda\left(H_{\boldsymbol{i}}\right) \geqq 0$ for all $1 \leqq i \leqq l$.

Lemma 17. Let $\pi$ be an irreducible representation of $\mathfrak{A}$ on a finite-dimensional space $V \neq\{0\}$. Then $\pi$ has a highest weight $\Lambda$ which is a dominant integral function. Also $V=\sum_{\lambda} V_{\lambda}$ where $\lambda$ runs over all the weights of $\pi$.

Since $K$ is algebraically closed and $\pi\left(H_{i}\right), 1 \leqq i \leqq l$, commute with each other it follows that we can find an element $\psi \in V(\psi \neq 0)$ such that $\psi$ is a common eigenvector of $\pi\left(H_{i}\right), 1 \leqq i \leqq l$. Therefore there exists a linear function $\lambda$ on $\mathfrak{h}$ such that $\pi(H) \psi=\lambda(H) \psi(H \in \mathfrak{h})$. Since $V$ is irreducible, $V=\pi(\mathfrak{l}) \psi$. Hence $V$ is spanned by $\pi(z(P, M, Q)) \psi$ for all $P, M$, and $Q$. But from Lemma $10, \pi(z(P, M, Q)) \psi$ has the weight $\lambda+$ rank $P$-rank $Q$. Therefore $V=\sum_{\mu} V_{\mu}$ where $\mu$ runs over all the weights of $\pi$. Since $\operatorname{dim} V$ is finite it follows from Lemma 1 that $\pi$ has only a finite number of weights. Now if $\varphi \in V_{\mu}$ then $\pi\left(X_{i}^{\nu}\right) \varphi \neq V_{\mu+\nu \alpha_{i}}$. Since $\mu+\nu \alpha_{i}, \nu=1,2, \cdots$, are all distinct linear functions on $\mathfrak{h}$ they cannot all be weights of $\pi$. Hence $\pi\left(X_{i}^{v}\right) \varphi=0$ for some $\nu$. Similarly we show that $\pi\left(Y_{i}^{\nu}\right) \varphi=0$ for some $\nu$. Since $V=\sum_{\mu} V_{\mu}$ it is clear that the hypotheses of Lemma 11 are fulfilled. Hence every weight of $\pi$ is an integral function. Since $\pi$ has only a finite number of weights it has a highest weight $\Lambda$. From Lemma $11, \sigma_{i} \Lambda$ is also a weight for every $i, 1 \leqq i \leqq l$. Since $\Lambda$ is highest, $\Lambda \geqq \sigma_{i} \Lambda=\Lambda-\Lambda\left(H_{i}\right) \alpha_{i}$. Hence $\Lambda\left(H_{i}\right) \geqq 0$, and $\Lambda$ is dominant.

Corollary. Every finite-dimensional irreducible representation of $\mathfrak{A}$ is equivalent to some $\pi_{\Delta}$ where $\Lambda$ is a dominant integral function on $\mathfrak{h}$.

This is an immediate consequence of Lemmas 16 and 17.

Let $\mathrm{g}$ be the smallest subspace of $\mathscr{A}$ which contains $X_{i}, Y_{i}, H_{i}, 1 \leqq i \leqq l$, and which is invariant under the adjoint representation of $\mathfrak{A}$. Let $\mathfrak{X}$ be the smallest subspace which contains $X_{i}, 1 \leqq i \leqq l$, and which is invariant under ad $X_{i}, 1 \leqq i \leqq l$. Similarly let $\mathfrak{V}$ be the smallest subspace containing $Y_{i}$, 
$1 \leqq i \leqq l$, and invariant under ad $Y_{i}, 1 \leqq i \leqq l$.

LEMmA 18. $\mathfrak{g}=\mathfrak{h}+\mathfrak{X}+\mathfrak{Y}$ and $(\operatorname{ad} z) w=[z, w]$ for any $z \in \mathfrak{g}$ and $w \in \mathfrak{A}$. Further if $z, w \in \mathfrak{g}$ then $[z, w] \in \mathfrak{g}$.

It is obvious that $\mathfrak{h}+\mathfrak{X}+\mathfrak{Y} \subset \mathfrak{g}$. Hence in order to prove the equality it is sufficient to show that $\mathfrak{h}+\mathfrak{X}+\mathfrak{Y}$ is invariant under ad $\mathfrak{l}_{0}$ where $\mathfrak{R}_{0}$ is the linear space spanned by $X_{i}, Y_{i}, H_{i}, 1 \leqq i \leqq l$. It is clear from its definition that $\mathfrak{X}$ is spanned by all elements of the form $\{\operatorname{ad} z(\phi, 0, P)\} X_{i}$. Put $z_{P}=z(\phi, 0, P)$ and $z=\left(\operatorname{ad} z_{P}\right) X_{i}$ for brevity. Then if $H \in \mathfrak{h}$,

$$
\begin{aligned}
(\operatorname{ad} H) z & =\left(\operatorname{ad}\left[H, z_{P}\right]\right) X_{i}+\left(\operatorname{ad} z_{P}\right)\left[H, X_{i}\right] \\
& =\lambda(H) z
\end{aligned}
$$

where $\lambda(H)=$ rank $P+\alpha_{i}$. This shows that $\mathfrak{X}$ is invariant under ad $H$. Now consider

$$
\left(\operatorname{ad} Y_{j}\right) z=\left(\operatorname{ad}\left[Y_{j}, z_{P}\right]\right) X_{i}+\left(\operatorname{ad} z_{P}\right)\left[Y_{j}, X_{i}\right] .
$$

Since $\left[Y_{j}, X_{i}\right]=-\delta_{i j} H_{i}$, it is clear that $\left(\operatorname{ad} z_{P}\right)\left[Y_{j}, X_{i}\right] \in \mathfrak{h}+\mathfrak{X}$. We claim further that $\left(\operatorname{ad}\left[Y_{j}, z_{P}\right]\right) X_{i} \in \mathfrak{h}+\mathfrak{X}$. If $|P|=0$ this is true. Hence we may assume $|P| \geqq 1$ and use induction on $|P|$. Then

$$
z_{P}=X_{k} z\left(\phi, 0, P^{\prime}\right)
$$

for some $k$ and $P^{\prime}$ such that $\left|P^{\prime}\right|=|P|-1$ and $1 \leqq k \leqq l$. Put $z\left(\phi, 0, P^{\prime}\right)=z_{P^{\prime}}$. Then

$$
\left[Y_{j}, z_{P}\right]=\left[Y_{j}, X_{k}\right] z_{P^{\prime}}+X_{k}\left[Y_{j}, z_{P^{\prime}}\right]=-\delta_{j k} H_{k} z_{P^{\prime}}+X_{k}\left[Y_{j}, z_{P^{\prime}}\right] .
$$

Therefore

$$
\left(\operatorname{ad}\left[Y_{j}, z_{P}\right]\right) X_{i}=-\delta_{j k}\left(\operatorname{ad} H_{k}\right)\left(\operatorname{ad} z_{P^{\prime}}\right) X_{i}+\left(\operatorname{ad} X_{k}\right)\left(\operatorname{ad}\left[Y_{j}, z_{P^{\prime}}\right]\right) X_{i \bullet}
$$

Clearly $\left(\operatorname{ad} z_{P^{\prime}}\right) X_{i} \in \mathfrak{X}$ and $\left(\operatorname{ad}\left[Y_{j}, z_{P^{\prime}}\right]\right) X_{i} \in \mathfrak{h}+\mathfrak{X}$ by induction hypothesis. Since $\mathfrak{h}+\mathfrak{X}$ is invariant under ad $H_{k}$ and ad $X_{k}$ the assertion follows. Hence $\left(\operatorname{ad} Y_{j}\right) z \in \mathfrak{h}+\mathfrak{X}$. Since $\mathfrak{X}$ is invariant under ad $X_{j}$ we have shown that $(\operatorname{ad} w) z \in \mathfrak{h}+\mathfrak{X}$ for any $w \in \Omega_{0}$ and $z \in \mathfrak{X}$. Similarly we prove that $(\operatorname{ad} w) z$ $\in \mathfrak{h}+\mathfrak{Y}$ for any $w \in \mathfrak{R}_{0}$ and $z \in \mathfrak{Y}$. Finally it is clear that if $w \in \mathfrak{R}_{0}$ and $H \in \mathfrak{h}$ then $(\operatorname{ad} w) H \in \mathfrak{X}+\mathfrak{Z}$. Hence it follows that $\mathfrak{h}+\mathfrak{X}+\mathfrak{Y}$ is invariant under ad $\mathfrak{R}_{0}$ and therefore $\mathfrak{g}=\mathfrak{h}+\mathfrak{X}+\mathfrak{Y}$.

Keeping to the above notation, let $z=\left(\operatorname{ad} z_{P}\right) X_{i}$ and $w \in \mathfrak{A}$. We claim that $(\operatorname{ad} z) w=[z, w]$. If $|P|=0, z=X_{i}$ and this is true. Hence again we may assume $|P| \geqq 1$ and use induction on $|P|$. Then as above $z_{P}=X_{k} z_{P}$, with $\left|P^{\prime}\right|=|P|-1$. Put $z^{\prime}=\left(\operatorname{ad} z_{P^{\prime}}\right) X_{i}$. Then

$$
z=\left(\operatorname{ad} z_{P}\right) X_{i}=\left(\operatorname{ad} X_{k} \text { ad } z_{P^{\prime}}\right) X_{i}=\left[X_{k}, z^{\prime}\right]
$$

Hence 


$$
\begin{aligned}
(\operatorname{ad} z) w & =\left(\operatorname{ad}\left[X_{k}, z^{\prime}\right]\right) w=\left[X_{k},\left(\operatorname{ad} z^{\prime}\right) w\right]-\left(\operatorname{ad} z^{\prime}\right)\left[X_{k}, w\right] \\
& =\left[X_{k},\left[z^{\prime}, w\right]\right]-\left[z^{\prime}\left[X_{k}, w\right]\right] \text { (by the induction hypothesis) } \\
& =\left[\left[X_{k}, z^{\prime}\right], w\right]=[z, w] .
\end{aligned}
$$

Our assertion is therefore proved. Hence by linearity $(\operatorname{ad} z) w=[z, w]$ for any $z \in \mathfrak{X}$. Similarly we prove that $(\operatorname{ad} z) w=[z, w]$ for any $z \in \mathfrak{Y}$ and $w \in \mathfrak{A}$. Finally if $H \in \mathfrak{h},(\operatorname{ad} H) w=[H, w]$. Hence $(\operatorname{ad} z) w=[z, w]$ for all $z \in \mathfrak{h}+\mathfrak{X}+\mathfrak{V}$ $=\mathfrak{g}$ and $w \in \mathfrak{A}$. Since $\mathfrak{g}$ is invariant under the adjoint representation it follows that $(\operatorname{ad} z) w \in \mathfrak{g}$ for any $z \in \mathfrak{A}$ and $w \in \mathfrak{g}$. Therefore if $z \in \mathfrak{g},[z, w]=(\operatorname{ad} z) w \in \mathfrak{g}$. This completes the proof of the lemma.

Let $\Lambda_{i}, 1 \leqq i \leqq l$, be the dominant integral functions on $\mathfrak{h}$ defined by $\Lambda_{i}\left(H_{j}\right)$ $=\delta_{i j}, 1 \leqq j \leqq l$. $\Lambda_{0}$ being any given dominant integral function on $\mathfrak{h}$, let $\pi$ denote the direct sum of the finite-dimensional representations $\pi_{\Delta_{i}}, 0 \leqq i \leqq l$. Then $\pi(\mathfrak{U})$ is a finite-dimensional associative algebra and therefore from Lemma $18, \pi(g)$ is a linear $\left(^{7}\right)$ Lie algebra.

Leмма 19. $\pi$ maps $\mathfrak{h}$ isomorphically on $\pi(\mathfrak{h})$. Further $\pi(\mathfrak{g})$ is a semisimple Lie algebra and $\pi(\mathfrak{h})$ is a Cartan subalgebra of $\pi(\mathfrak{g})$.

Let $V$ be the representation space of $\pi$ and let $H \in \mathfrak{h}(H \neq 0)$. Since $\Lambda_{i}$, $1 \leqq i \leqq l$, are linearly independent, $\Lambda_{i}(H) \neq 0$ for some $i$. Since $\Lambda_{i}$ is the highest weight of $\pi_{\Lambda_{i}}$ it is also a weight of $\pi$. Hence we can choose $\psi \in V_{\Lambda_{i}}(\psi \neq 0)$. Then $\pi(H) \psi=\Lambda_{i}\left(H_{i}\right) \psi \neq 0$. Hence $\pi(H) \neq 0$ and this shows that $\pi$ maps $\mathfrak{h}$ isomorphically.

Let $\mathfrak{B}$ be the kernel of $\pi$ and $(\mathfrak{S}$ the set of all elements $z \in \mathfrak{g}$ such that $[H, z] \in \mathfrak{B}$ for all $H \in \mathfrak{h}$. Clearly $\mathfrak{S}$ is invariant under ad $H(H \in \mathfrak{h})$. Let $z \in \mathfrak{C}$. From Lemma 1 every homogeneous component of $z$ belongs to $\mathfrak{c}$. Let $z_{\lambda}$ be such a component of $\operatorname{rank} \lambda$. Then $\left[H, z_{\lambda}\right]=\lambda(H) z_{\lambda} \in \mathfrak{B}$. If $\lambda \neq 0, \lambda(H) \neq 0$ for some $H \in \mathfrak{h}$. Hence $z_{\lambda} \in \mathfrak{B}$ and $\pi\left(z_{\lambda}\right)=0$. On the other hand let us now suppose that $\lambda=0$. We have seen above that $\mathfrak{X}$ is spanned by suitable homogeneous elements of rank greater than 0 and $\mathfrak{Y}$ by similar elements of rank less than 0 . Since $z_{\lambda} \in \mathfrak{g}=\mathfrak{h}+\mathfrak{X}+\mathfrak{Y}$ it follows that $z_{\lambda} \in \mathfrak{h}$. Therefore since $z=\sum_{\lambda} z_{\lambda}, \pi(z)=\pi\left(z_{0}\right) \in \pi(\mathfrak{h})$ and so $\pi(\mathfrak{C}) \subset \pi(\mathfrak{h})$. This shows that $\pi(\mathfrak{h})$ is a maximal abelian subalgebra of $\pi(\mathfrak{g})$. In particular the center of $\pi(\mathfrak{g})$ is contained in $\pi(\mathfrak{h})$. Let $\pi(H) \quad(H \in \mathfrak{h})$ belong to the center of $\pi(\mathfrak{g})$. Then $\left[\pi(H), \pi\left(X_{i}\right)\right]=\pi\left(\left[H, X_{i}\right]\right)=\alpha_{i}(H) \pi\left(X_{i}\right)=0$. But $\left[\pi\left(X_{i}\right), \pi\left(Y_{i}\right)\right]=\pi\left(H_{i}\right) \neq 0$ as we saw above. Hence $\pi\left(X_{i}\right) \neq 0$. Therefore $\alpha_{i}(H)=0,1 \leqq i \leqq l$. Since $\alpha_{i}$ are linearly independent this implies that $H=0$. Hence the center of $\pi(g)$ is $\{0\}$.

Now for each $i, 0 \leqq i \leqq l, \pi_{\Lambda_{i}}$ is an irreducible representation of $\mathfrak{A}$. Since $1, X_{j}, Y_{j}, H_{j}, 1 \leqq j \leqq l$, generate $\mathfrak{A}$ it follows that $\pi_{\Lambda_{i}}(\mathfrak{g})$ is an irreducible set of linear transformations, $0 \leqq i \leqq l$. Since $\pi$ is the direct sum of $\pi_{\Delta_{i}}, 0 \leqq i \leqq l$,

$\left({ }^{7}\right)$ A Lie algebra consisting of endomorphisms of a finite-dimensional vector space with the usual bracket operation $[A, B]=A B-B A$ is called a linear Lie algebra. 
$\pi(\mathrm{g})$ is a fully reducible set of endomorphisms of $V$. Since the center of $\pi(\mathfrak{g})$ is $\{0\}$ it follows (see Chevalley [5]) that $\pi(\mathfrak{g})$ is a semisimple Lie algebra.

Since $\pi$ maps $\mathfrak{h}$ isomorphically we can regard every linear function $\lambda$ on $\mathfrak{h}$ also as a linear function on $\pi(\mathfrak{h})$ by setting $\lambda(\pi(H))=\lambda(H)(H \in \mathfrak{h})$. In particular therefore $\alpha_{i}, 1 \leqq i \leqq l$, are now linear functions also on $\pi(\mathfrak{h})$. We shall now show that $\pi(\mathfrak{h})$ is a Cartan subalgebra of $\pi(\mathfrak{g})$ and $\alpha_{i}, 1 \leqq i \leqq l$, is a fundamental system of roots of $\pi(\mathfrak{g})$ with respect to $\pi(\mathfrak{h})$.

For a fixed $z \in \mathfrak{A}$ consider the linear mapping $w \rightarrow \pi((\operatorname{ad} z) w)$ of $\mathfrak{A}$ into $\pi(\mathfrak{H})$. Since the kernel $\mathfrak{B}$ of $\pi$ is an ideal, it is invariant under the adjoint representation of $\mathfrak{A}$. Hence $\pi(w)=0$ implies $\pi((\operatorname{ad} z) w)=0$. Therefore we get a linear mapping $\pi(w) \rightarrow \pi((\operatorname{ad} z) w)$ of $\pi(\mathfrak{H})$ into itself, which we denote by $\rho^{\prime}(z)$. Now

$$
\begin{aligned}
\rho^{\prime}\left(z_{1} z_{2}\right) \pi(w) & =\pi\left(\operatorname{ad}\left(z_{1} z_{2}\right) w\right)=\pi\left(\left(\operatorname{ad} z_{1}\right)\left(\operatorname{ad} z_{2}\right) w\right) \\
& =\rho^{\prime}\left(z_{1}\right) \rho^{\prime}\left(z_{2}\right) \pi(w) \quad\left(z_{1}, z_{2}, w \in \mathfrak{U}\right) .
\end{aligned}
$$

Hence $\rho^{\prime}$ is a representation of $\mathfrak{A}$. Since $\mathfrak{g}$ is invariant under the adjoint representation of $\mathfrak{A},(\mathrm{ad} z) w \in \mathfrak{g}$ if $w \in \mathfrak{g}$ and $z \in \mathfrak{A}$. Hence $\pi(\mathfrak{g})$ is invariant under $\rho^{\prime}(\mathfrak{A})$. Let $\rho$ be the representation of $\mathfrak{A}$ induced by $\rho^{\prime}$ on $\pi(\mathfrak{g})$. Then

$$
\rho(z) \pi(w)=\pi((\operatorname{ad} z) w)=\pi([z, w])=[\pi(z), \pi(w)] \quad(z, w \in \mathfrak{g})
$$

from Lemma 18. Since $\pi(\mathfrak{g})$ is semisimple its adjoint representation is fully reducible. Hence $\rho(\mathfrak{g})$ is a fully reducible set of endomorphisms of $\pi(\mathfrak{g})$. Since $\rho(\mathfrak{A})$ is generated by $\rho(\mathfrak{g})$ and 1 , it follows that $\rho$ is a fully reducible representation. Let $U=\pi(\mathfrak{g})$. Applying Lemma 17 to each irreducible component of $\rho$ we immediately get $U=\sum_{\lambda} U_{\lambda}$ where $\lambda$ runs over all weights of $\rho$. This shows that $\rho(H)$ is semisimple $\left.{ }^{8}\right)$ for each $H \in \mathfrak{h}$. But $\rho(H)=\operatorname{ad} \pi(H)$ where $\pi(z) \rightarrow \operatorname{ad} \pi(z)(z \in \mathfrak{g})$ denotes the adjoint representation of $\pi(\mathfrak{g})$. Hence we have shown that $\pi(\mathfrak{h})$ is a maximal abelian algebra of $\pi(\mathfrak{g})$ every element of which is mapped on a semisimple endomorphism under the adjoint representation of $\pi(\mathfrak{g})$. This proves that $\pi(\mathfrak{h})$ is a Cartan subalgebra of $\pi(\mathfrak{g})$.

Lemma 20. $\alpha_{i}, 1 \leqq i \leqq l$, is a fundamental system of roots of $\pi(\mathrm{g})$ with respect to $\pi(\mathfrak{h})$. Also $\operatorname{dim} \pi(\mathfrak{g})=l+g$ where $g$ is the number of distinct functions of the form $\sigma \alpha_{i}(\sigma \in W, 1 \leqq i \leqq l)$.

We keep to the above notation. We have seen that $U=\sum_{\lambda} U_{\lambda}$ where $\lambda$ runs over all weights of $\rho$. Notice that a nonzero weight of $\rho$ is exactly the same thing as a root of $\pi(\mathfrak{g}) . \lambda$ being a weight of $\rho$ choose a $z \in \mathfrak{g}$ such that $\pi(z) \in U_{\lambda}(\pi(z) \neq 0)$. Then $\rho(H) \pi(z)=[\pi(H), \pi(z)]=\lambda(H) \pi(z)$. Hence $[H, z]$ $-\lambda(H) z \in \mathfrak{B}$ for all $H \in \mathfrak{h}$. Let $z_{\mu}$ be the homogeneous component of $z$ of rank $\mu$. Then the corresponding component of $[H, z]-\lambda(H) z$ is $\left[H, z_{\mu}\right]-\lambda(H) z_{\mu}$

(8) An endomorphism $A$ of a finite-dimensional vector space $V$ is called semisimple if $V$ is fully reducible under $A$. 
$=\{\mu(H)-\lambda(H)\} z_{\mu}$. Since $\mathfrak{B}$ is invariant under ad $\mathfrak{h}$ it follows from Lemma 1 that $\{\mu(H)-\lambda(H)\} z_{\mu} \in \mathfrak{B}$ for all $H \in \mathfrak{h}$. If $\mu \neq \lambda$ we can find an $H \in \mathfrak{h}$ such that $\mu(H) \neq \lambda(H)$. Hence $z_{\mu} \in \mathfrak{B}$. Therefore $\pi(z)=\pi\left(z_{\lambda}\right) \neq 0$. Since $\mathfrak{g}$ is invariant under ad $\mathfrak{h}$ and $z \in \mathfrak{g}$ it follows again by Lemma 1 that $z_{\lambda} \in \mathfrak{g}$. Since $\mathfrak{g}=\mathfrak{h}+\mathfrak{X}+\mathfrak{Y}$ it is clear that $\mathfrak{g} \cap \mathfrak{A}_{\lambda}=\{0\}$ unless $\lambda=d_{1} \alpha_{1}+\cdots+d_{l} \alpha_{l}$ where $d_{i}$ are integers which are either all greater than or equal to 0 or all less than or equal to 0 . But $z_{\lambda} \in g$ and $z_{\lambda} \neq 0$. Hence $\lambda$ must be of the above form. Finally $\pi\left(X_{i}\right) \in U_{\alpha_{i}}$ and we have already seen in the proof of Lemma 19 that $\pi\left(X_{i}\right) \neq 0$. Hence $\alpha_{i}, 1 \leqq i \leqq l$, are roots of $\pi(\mathfrak{g})$. This proves that $\alpha_{i}, 1 \leqq i \leqq l$, is a fundamental system of roots of $\pi(\mathfrak{g})$.

Since $\pi\left(X_{i}\right) \neq 0$ and similarly $\pi\left(Y_{i}\right) \neq 0$, they are the root elements in $\pi(\mathfrak{g})$ corresponding to the roots $\alpha_{i}$ and $-\alpha_{i}$ respectively. Since $\left[\pi\left(X_{i}\right)\right.$, $\left.\pi\left(Y_{i}\right)\right]=\pi\left(H_{i}\right)$ it follows that if $\sigma_{j}^{\prime}$ is the Weyl reflexion in $\pi(\mathrm{g})$ with respect to $\alpha_{j}^{\prime}$,

$$
\sigma_{j}^{\prime} \alpha_{i}=\alpha_{i}-2 \frac{\alpha_{i}\left(H_{j}\right)}{\alpha_{j}\left(H_{j}\right)} \alpha_{j}=\sigma_{j} \alpha_{i}
$$

since $\alpha_{j}\left(H_{j}\right)=2$. Hence the Weyl matrix of $\pi(\mathfrak{g})$ with respect to the fundamental system $\left\{\alpha_{1}, \cdots, \alpha_{l}\right\}$ is $\left(a_{i j}\right), 1 \leqq i, j \leqq l$, and the Weyl group of $\pi(\mathfrak{g})$ is $W$. Therefore $\pi(\mathfrak{g})$ has exactly $g$ distinct roots. Since $\operatorname{dim} \pi(\mathfrak{h})=l$, $\operatorname{dim} \pi(\mathfrak{g})=l+g$.

Now consider the special case when $\Lambda_{0}=0$. As remarked earlier (cf. p. 43), in this case $\pi_{\Lambda_{0}}(\mathfrak{g})=\{0\}$. Let $\bar{\pi}$ be the direct sum of $\pi_{\Delta_{i}}, 1 \leqq i \leqq l$. Then clearly $\pi(\mathfrak{g})$ is isomorphic to $\bar{\pi}(\mathfrak{g})$ under the mapping $\pi(z) \leftrightarrow \bar{\pi}(z)(z \in \mathfrak{g})$. We put $\mathfrak{l}=\bar{\pi}(\mathfrak{g})$. Again we may regard $\alpha_{i}$ as linear functions on $\bar{\pi}(\mathfrak{h})$ in the obvious way. Then $\mathfrak{R}$ is a semisimple Lie algebra with the fundamental system of roots $\alpha_{i}, 1 \leqq i \leqq l$, with respect to the Cartan subalgebra $\bar{\pi}(\mathfrak{h})$ and $\operatorname{dim} \mathfrak{R}$ $=\operatorname{dim} \pi(g)=l+g$. Returning to the general case when $\Lambda_{0}$ is arbitrary, it is obvious that $\pi(z) \rightarrow \bar{\pi}(z)(z \in g)$ is a homomorphism of $\pi(\mathfrak{g})$ onto $\mathbb{R}$. But since both $\pi(\mathfrak{g})$ and $\mathfrak{R}$ have the same dimension $l+g$, this must be an isomorphism. Also $\pi(z) \rightarrow \pi_{\Lambda_{0}}(z) \quad(z \in \mathfrak{g})$ is evidently a representation of $\pi(\mathfrak{g})$. Therefore $\bar{\pi}(z) \rightarrow \pi_{\Lambda_{0}}(z)(z \in g)$ is a representation of $\mathfrak{l}$ whose highest weight is $\Lambda_{0}$. The proof of Theorem 1 is therefore complete.

We now consider the question of the uniqueness of the Lie algebra whose existence is asserted in Theorem 1 . Let $\overline{\mathfrak{Q}}$ be any semisimple Lie algebra with a Cartan subalgebra $\overline{\mathfrak{h}}$ satisfying the requirements of the theorem. Let $\bar{X}_{i}, \bar{Y}_{i}$ be the root elements of $\overline{\mathfrak{l}}$ corresponding to the roots $\alpha_{i}$ and $-\alpha_{i}$, $1 \leqq i \leqq l$. Put $\left[\bar{X}_{i}, \bar{Y}_{i}\right]=\bar{H}_{i}$. Since $\alpha_{i}$ is a fundamental system of roots, $\alpha_{i}-\alpha_{j}$ $(i \neq j)$ is not a root. Hence $\left[\bar{X}_{i}, \bar{Y}_{j}\right]=0$. By multiplying $\bar{X}_{i}$ with an element $c \in K(c \neq 0)$ we may arrange that $\alpha_{i}\left(\bar{H}_{i}\right)=2,1 \leqq i \leqq l$. Then it follows that

$$
\sigma_{i} \alpha_{j}=\alpha_{j}-\frac{2 \alpha_{j}\left(\bar{H}_{i}\right)}{\alpha_{i}\left(\bar{H}_{i}\right)} \alpha_{i}=\alpha_{j}+a_{j i} \alpha_{i} .
$$


Hence $\alpha_{j}\left(\bar{H}_{i}\right)=\alpha_{j}\left(H_{i}\right), 1 \leqq i, j \leqq l$. Let $\rho^{\prime}$ denote the representation of the free algebra $\mathfrak{A}^{\prime}$ on $\overline{\mathfrak{Q}}$ defined uniquely by the equations

$$
\rho^{\prime}(1)=I, \quad \rho^{\prime}\left(X_{i}^{\prime}\right)=\operatorname{ad} \bar{X}_{i}, \rho^{\prime}\left(Y_{i}^{\prime}\right)=\operatorname{ad} \bar{Y}_{i}, \rho^{\prime}\left(H_{i}^{\prime}\right)=\operatorname{ad} \bar{H}_{i}, \quad 1 \leqq i \leqq l,
$$

where $I$ is the identity mapping of $\mathbb{R}$ and $\bar{X} \rightarrow$ ad $\bar{X}(\bar{X} \in \overline{\mathfrak{R}})$ denotes the adjoint representation of $\overline{\mathfrak{Q}}$. It is easily seen that the kernel of $\rho^{\prime}$ contains the ideal $\mathfrak{U}^{\prime}$ and so $\rho^{\prime}$ actually defines a representation $\bar{\rho}$ of $\mathfrak{A}$ on $\overline{\mathfrak{R}}$. Since $\overline{\boldsymbol{\Omega}}$ is semisimple its adjoint representation is fully reducible. Hence $\bar{\rho}$ is also fully reducible. From the corollary to Lemma 17, every finite-dimensional irreducible representation of $\mathfrak{A}$ is equivalent to $\pi_{\Delta}$ for some dominant integral function $\Lambda$. But we have seen above that $\bar{\pi}(z) \rightarrow \pi_{\Lambda}(z)(z \in \mathfrak{g})$ is a representation of $\mathfrak{R}$. Hence it is clear that $\bar{\pi}(z) \rightarrow \bar{\rho}(z)(z \neq \mathfrak{g})$ is also a representation of $\mathfrak{R}$. We denote this representation by $\theta$. Then $\theta(\Omega)$ is a linear Lie algebra and since $\operatorname{dim} \mathfrak{l}=l+g, \operatorname{dim} \theta(\mathfrak{R}) \leqq l+g$. But clearly $\theta(\overline{\mathfrak{l}}) \supset$ ad $\overline{\mathfrak{l}}$ and $\operatorname{dim}(\operatorname{ad} \overline{\mathfrak{l}})$ $=\operatorname{dim} \bar{\Omega}=l+g$ since $g$ depends only on the group $W$ which is the same for both $\overline{\mathfrak{R}}$ and $\mathfrak{R}$. Hence $\theta(\mathfrak{R})=$ ad $\overline{\mathfrak{R}}$ and $\theta$ must be an isomorphism. Therefore $\mathbb{R} \cong$ ad $\overline{\mathbb{Q}} \cong \overline{\mathbb{R}}$. This shows that $\mathbb{R}$ is uniquely determined up to an isomorphism.

Also notice that if $\theta$ is any representation of $\&$ on $V$ then $\theta$ defines uniquely a representation $\varphi$ of $\mathfrak{A}$ by the rule

$$
\varphi(1)=I, \quad \varphi(z)=\theta(\bar{\pi}(z))
$$

where $I$ is the identity mapping of $V$ and $z$ is any one of the elements $X_{i}, Y_{i}$, $H_{i}, 1 \leqq i \leqq l$. We claim that

$$
\varphi(z)=\theta(\bar{\pi}(z))
$$

for all $z \in \mathfrak{g}$. This is obvious if $z \in \mathfrak{h}$. Now suppose $z \in \mathfrak{X}$. It is sufficient to consider the case when $z=\left(\operatorname{ad} z_{P}\right) X_{i}$ where $z_{P}=z(\phi, 0, P)$. Again if $|P|=0$ the statement is true. Hence we may assume that $|P| \geqq 1$ and use induction on $|P|$. Let $z_{P}=X_{k} z_{P^{\prime}}$, where $z_{P^{\prime}}=z\left(\phi, 0, P^{\prime}\right),\left|P^{\prime}\right|=|P|-1,1 \leqq k \leqq l$. Then

$$
z=\left[X_{k}, z^{\prime}\right]
$$

where $z^{\prime}=\left(\operatorname{ad} z_{P^{\prime}}\right) X_{i}$. Hence

$$
\begin{aligned}
\varphi(z) & =\varphi\left(\left[X_{k}, z^{\prime}\right]\right)=\left[\varphi\left(X_{k}\right), \varphi\left(z^{\prime}\right)\right] \\
& =\left[\theta\left(\bar{\pi}\left(X_{k}\right)\right), \theta\left(\bar{\pi}\left(z^{\prime}\right)\right)\right]
\end{aligned}
$$

by induction hypothesis. Therefore

$$
\begin{aligned}
\varphi(z) & =\theta\left(\left[\bar{\pi}\left(X_{k}\right), \bar{\pi}\left(z^{\prime}\right)\right]\right)=\theta\left(\bar{\pi}\left(\left[X_{k}, z^{\prime}\right]\right)\right) \\
& =\theta(\bar{\pi}(z)) .
\end{aligned}
$$

Similarly if $z \in \mathfrak{Y}$. Since $\mathfrak{g}=\mathfrak{h}+\mathfrak{X}+\mathfrak{Y}$ the assertion follows. In particular if $\theta$ is irreducible the same is true of $\varphi$ and the weights of $\varphi$ are the same as those of $\theta$. Hence if $\theta$ is irreducible and finite-dimensional and $\Lambda$ is the highest weight 
of $\theta$ then it follows from Lemma 16 that $\varphi$ is equivalent to $\pi_{\Delta}$. Hence $\varphi$ and therefore $\theta$ is uniquely determined up to equivalence, and the multiplicity of $\Lambda$ in $\theta$ is 1 . Thus we have proved the following theorem.

THEOREM 2. The Lie algebra $\mathbb{R}$ of Theorem 1 is unique within isomorphism. Also the irreducible representation $\rho$ of $\mathbb{R}$ with the highest weight $\Lambda_{0}$ is uniquely determined within equivalence and the multiplicity of $\Lambda_{0}$ in $\rho$ is 1 .

Another way of stating the first part of Theorem 2 is to say that two semisimple Lie algebras with isomorphic root diagrams are isomorphic. In this form this result was first proved by Weyl [12]. The uniqueness of the representation $\rho$ is due to Cartan [2].

Finally we shall prove a result on the degree of the representation $\rho$. The exact formula for this degree has been obtained by Weyl [12] by using transcendental methods.

Theorem 3. Let $\mathfrak{R}, \mathfrak{h}, \alpha_{i}, 1 \leqq i \leqq l$, and $\rho$ be as in Theorems 1 and 2 and let $d_{\rho}$ be the degree of the representation $\rho$. Then

$$
\coprod_{1 \leqq i \leqq l}\left(\frac{2 \Lambda_{0}\left(H_{\alpha_{i}}\right)}{\alpha_{i}\left(H_{\alpha_{i}}\right)}+1\right) \leqq d_{\rho} \leqq \prod_{\alpha>0}\left(\frac{2 \Lambda_{0}\left(H_{\alpha}\right)}{\alpha\left(H_{\alpha}\right)}+1\right)
$$

where $\alpha$ denotes any root of $\mathfrak{R}$ with respect to $\mathfrak{h}$.

Let $\alpha_{i}, 1 \leqq i \leqq r$, be the set of all roots of $\mathfrak{R}$ which are greater than 0 and let $x_{i} \neq 0$ and $y_{i} \neq 0$ be root elements in $\&$ corresponding to the roots $\alpha_{i}$ and $-\alpha_{i}$ respectively, $1 \leqq i \leqq r$. Also let $H_{i}=\left[x_{i}, y_{i}\right], 1 \leqq i \leqq r$. (Notice that $H_{i}$ corresponds to $\bar{\pi}\left(H_{i}\right), 1 \leqq i \leqq l$, in our earlier notation.) Then $x_{i}, y_{i}, H_{j}, 1 \leqq i$ $\leqq r, 1 \leqq j \leqq l$, form a base for $\mathfrak{R}$. Let $\mathfrak{U}$ be the universal enveloping algebra $\left({ }^{9}\right)$ of $\mathcal{R}$ and let $V$ be the representation space of $\rho$. Then $\rho$ defines a representation $\pi$ of $\mathfrak{U}$ on $V$ by the rule

$$
\pi(1)=I, \quad \pi(x)=\rho(x)
$$

where $I$ is the identity mapping of $V$. Let $\psi \neq 0$ be an element in $V$ which belongs to the weight $\Lambda_{0}$. Let $\mathfrak{M}$ be the left ideal in $\mathfrak{U}$ consisting of all elements $z \in \mathfrak{U}$ such that $\pi(z) \psi=0$. Since $V$ is irreducible under $\rho$ and therefore under $\pi, \mathfrak{M}$ is a maximal left ideal. Let $\pi^{*}$ denote the natural representation and $z \rightarrow z^{*}$ the natural mapping of $\mathfrak{U}$ on $\mathfrak{U}^{*}=\mathfrak{U} / \mathfrak{M}$. It is easily verified that $\pi^{*}$ is equivalent to $\pi$ under the isomorphism $z^{*} \leftrightarrow \pi(z) \psi(z \in \mathfrak{U})$ of $\mathfrak{U}^{*}$ with $V$. Hence instead of $\rho$ we may consider the representation $\pi^{*}$. Notice that since $\Lambda_{0}$ is the highest weight of $\rho, \Lambda_{0}+\alpha_{i}$ is not a weight for any $i, 1 \leqq i \leqq r$. Hence $\pi\left(x_{i}\right) \psi=0$ and $x_{i} \in \mathfrak{M}, 1 \leqq i \leqq r$. Also since $\pi(H) \psi=\Lambda_{0}(H) \psi, H-\Lambda_{0}(H) \in \mathfrak{M}$ for all $H \in \mathfrak{h}$.

( $\left.{ }^{9}\right)$ This concept is due to Birkhoff [1] and Witt [14]. However we follow the definition given in [9]. We shall always assume that the Lie algebra is naturally imbedded in its universal enveloping algebra. 
We now introduce the notion of a rank in $\mathfrak{U}$ exactly as we did it in $\mathfrak{A} . \lambda$ being any linear function on $\mathfrak{h}$ we say that an element $z \in \mathfrak{U}$ is of rank $\lambda$ if $[H, z]=\lambda(H) z(H \in \mathfrak{h})$.

By multiplying $x_{i}$ by an element $c_{i} \in K\left(c_{i} \neq 0\right)$ we can arrange that $\alpha_{i}\left(H_{i}\right)=2,1 \leqq i \leqq 2$. We shall suppose that this has been done. Now we assert that the elements $y_{1}^{m_{1}} y_{2}^{m_{2}} \ldots y_{l}^{m_{l}}, 0 \leqq m_{i} \leqq \Lambda_{0}\left(H_{i}\right), \quad 1 \leqq i \leqq l$, are linearly independent $\left({ }^{10}\right) \bmod \mathfrak{M}$. Notice that $y_{1}^{m_{1}} y_{2}^{m_{2}} \ldots y_{l}^{m_{l}}$ is of rank $-\left(m_{1} \alpha_{1}+\cdots+m_{l} \alpha_{l}\right)$. Since $\alpha_{i}, 1 \leqq i \leqq l$, are linearly independent, all these linear functions are distinct. Since $H-\Lambda_{0}(H) \in \mathfrak{M}(H \in \mathfrak{h}), \mathfrak{M}$ is invariant under ad $\mathfrak{h}$. Hence in view of Lemma 1 it is sufficient to prove that $y_{1}^{m_{1}} y_{2}^{m_{2}} \cdots y_{l}^{m_{l}}$ $\notin \mathfrak{M}$ for $0 \leqq m_{i} \leqq \Lambda_{0}\left(H_{i}\right), 1 \leqq i \leqq l$. Let $M=m_{1}+m_{2}+\cdots+m_{l}$. If $M=0$, $y_{1}^{m_{1}} \cdots y_{l}^{m_{l}}=1$ and the assertion is true since $1 \notin \mathfrak{M}$. Hence we may assume $M \geqq 1$ and use induction on $M$. Let $i$ be the largest index such that $m_{i} \neq 0$. Put $z=1$ if $i=1$ or $z=y_{1}^{m_{1}} y_{2}^{m_{2}} \cdots y_{i-1}^{m_{i-1}}$ if $i>1$. Then we have to consider the term $z y_{i}^{m_{i}}$. Notice that $\left[x_{i}, y_{j}\right]=0$ if $i \neq j, 1 \leqq i, j \leqq l$. This follows from the fact that $\alpha_{i}, 1 \leqq i \leqq l$, is a fundamental system and so $\alpha_{i}-\alpha_{j}$ is not a root, $1 \leqq i, j \leqq l(i \neq j)$. Hence from Lemma 6 ,

$$
\begin{aligned}
x_{i} z y_{i}^{m_{i}} & =z x_{i} y_{i}^{m_{i}}=z\left[x_{i}, y_{i}^{m_{i}}\right]+z y_{i}^{m_{i}} x_{i} \\
& =m_{i} z y_{i}^{m_{i}-1}\left(H_{i}-m_{i}+1\right)+z y_{i}^{m_{i}} x_{i} \\
& \equiv m_{i} z y_{i}^{m_{i}-1}\left(\Lambda_{0}\left(H_{i}\right)-m_{i}+1\right) \bmod \mathfrak{M} .
\end{aligned}
$$

Now $0<m_{i} \leqq \Lambda_{0}\left(H_{i}\right)$. Hence $m_{i}\left(\Lambda_{0}\left(H_{i}\right)-m_{i}+1\right) \neq 0$. Also by induction hypothesis $z y_{i}^{m_{i}-1} \in \mathfrak{M}$. Hence $x_{i} z y_{i}^{m_{i}} \in \mathfrak{M}$. Since $\mathfrak{M}$ is a left ideal, it follows that $z y_{i}^{m_{i}} \notin \mathfrak{M}$. Therefore our assertion is proved. Since the elements $y_{1}^{m_{1}} y_{2}^{m_{2}} \cdots y_{l}^{m_{l}}$, $0 \leqq m_{i} \leqq \Lambda_{0}\left(H_{i}\right), 1 \leqq i \leqq l$, are $\prod_{1 \leqq i \leqq l}\left(\Lambda_{0}\left(H_{i}\right)+1\right)$ in number and since they are linearly independent $\bmod \mathfrak{M}$, it follows that

$$
\operatorname{dim} \mathfrak{U}^{*} \geqq \prod_{1 \leqq i \leqq l}\left(\Lambda_{0}\left(H_{i}\right)+1\right) .
$$

Hence the first inequality of the theorem is proved.

Now we come to the second inequality. For brevity write

$$
z(q, m, p)=y_{1}^{q_{1}} y_{2}^{q_{2}} \cdots y_{r}^{q_{r}} H_{1}^{m_{1}} H_{2}^{m_{2}} \cdots H_{l}^{m_{l}} x_{1}^{p_{1}} \cdots x_{r}^{p_{r}}
$$

where $q_{i}, p_{i}, m_{j} \geqq 0,1 \leqq i \leqq r, 1 \leqq j \leqq l$, and $q, m, p$ denote the corresponding sets of integers. Also put $|q|=q_{1}+\cdots+q_{r},|p|=p_{1}+p_{2}+\cdots+p_{r},|m|$ $=m_{1}+\cdots+m_{l}$ and $s=|q|+|m|+|p|$. We shall write 0 for the set $p$ all of whose elements are zero. Similarly for $q$ and $m$. It is known (see for example [9]) that the elements $z(q, m, p)$ for all $q, m$, and $p$ span $\mathfrak{u}$. Let $U$ be the subspace of $\mathfrak{U}$ spanned by all elements of the form $y_{1}^{q_{1}} y_{2}^{q_{2}} \cdots y_{r}^{q_{r}}, 0 \leqq q_{i} \leqq \Lambda_{0}\left(H_{i}\right)$, $1 \leqq i \leqq r$. We shall prove that $\mathfrak{U}=\mathfrak{M}+U$. First we claim that if $q_{j}>\Lambda_{0}\left(H_{j}\right)$ then

(10) We use the convention that $z^{m}=1$ for any $z \in \mathfrak{U}$ if $m=0$. 
$y_{j}^{q_{j}} \in \mathfrak{M}, 1 \leqq j \leqq r$. For otherwise $\left(y_{j}^{q_{i}}\right)^{*} \neq 0$. Since $y_{j}^{q_{j}}$ has the rank $-q_{j} \alpha_{j}$ it follows that $\left(y_{j}^{q_{j}}\right)^{*}$ is homogeneous of weight $\Lambda_{0}-q_{j} \alpha_{j}$. Since $\left(y_{j}^{q_{i}}\right)^{*} \neq 0, \Lambda_{0}-q_{j} \alpha_{j}$ is a weight of $\pi^{*}$ and therefore of $\rho$. Let $\sigma_{j}$ denote the Weyl reflexion with respect to the root $\alpha_{j}$. Then $\left.{ }^{11}\right) \sigma_{j}\left(\Lambda_{0}-q_{j} \alpha_{j}\right)$ must also be a weight of $\rho$. But

$$
\sigma_{j}\left(\Lambda_{0}-q_{j} \alpha_{j}\right)=\Lambda_{0}-\Lambda_{0}\left(H_{j}\right) \alpha_{i}+q_{j} \alpha_{j}>\Lambda_{0}
$$

since $q_{j}>\Lambda_{0}\left(H_{j}\right)$. This contradicts our hypothesis that $\Lambda_{0}$ is the highest weight of $\rho$. Hence $y_{j}^{q_{j}} \in \mathfrak{M}$.

In order to prove that $\mathfrak{U}=\mathfrak{M}+U$ it is sufficient to show that $z(q, m, p)$ $\in \mathfrak{M}+U$ for all $q, m, p$. If $s=|q|+|m|+|p|=0$ this is true since $1 \in U$. Hence we may assume $s \geqq 1$ and use induction on $s$. Now if $|p|>0$ it is clear that $z(q, m, p) \in \mathfrak{M}$ since $x_{i} \in \mathfrak{M}, 1 \leqq i \leqq r$. Similarly since $H_{i}-\Lambda_{0}\left(H_{i}\right) \in \mathfrak{M}$, it follows that $z(q, m, 0) \equiv c z(q, 0,0) \bmod \mathfrak{M}(c \in K)$. Hence we need consider only elements of the form $z(q, 0,0)$ with $|q|=s$. If $q_{j} \leqq \Lambda_{0}\left(H_{j}\right)$ for all $j$, it is obvious from the definition of $U$ that $z(q, 0,0) \in U$. Hence we may assume that $q_{j}>\Lambda_{0}\left(H_{j}\right)$ for some $j$. Choose the greatest such $j$. If $j=r$ then $z(q, 0,0)$ $\in \mathfrak{M}$ since $\mathfrak{M}$ is a left ideal and we have seen above that $y_{r}^{q_{r}} \in \mathfrak{M}$ if $q_{r}>\Lambda_{0}\left(H_{r}\right)$. Hence we may suppose that $j<r$. Put $z_{0}=1$ if $j=1$ and $z_{0}=y_{1}^{q_{1}} \cdots y_{j-1}^{g_{j-1}}$ if $j>1$. Then since $y_{j}^{g_{j}} \in \mathfrak{M}$,

$$
z(q, 0,0) \equiv z_{0}\left[y_{i}^{q_{j}}, y_{j+1}^{q_{j+i}} \cdots y_{r}^{q_{r}}\right] \bmod \mathfrak{M}
$$

But it is known (see [9]) that $z_{0}\left[y_{j}^{q_{i}}, y_{j+1}^{q_{j}+1} \ldots y_{r}^{q_{r}}\right]=z^{\prime}$ can be written as a linear combination of $\boldsymbol{z}\left(q^{\prime}, m, p\right)$ with $\left|q^{\prime}\right|+|m|+|p|<s$. Hence it follows by induction hypothesis that $z^{\prime} \in \mathfrak{M}+U$. Therefore $z(q, 0,0) \in \mathfrak{M}+U$ and our assertion is proved.

Since $\mathfrak{M}+U=\mathfrak{U}$ it is clear that $\operatorname{dim} \mathfrak{u}^{*} \leqq \operatorname{dim} U$. Since $U$ is spanned by the $\prod_{1 \leqq i \leqq r}\left(\Lambda_{0}\left(H_{i}\right)+1\right)$ elements $y_{1}^{q_{1}} y_{2}^{q_{2}} \cdots y_{r}^{q_{r}}, 0 \leqq q_{i} \leqq \Lambda_{0}\left(H_{i}\right), 1 \leqq i \leqq r$, it follows that

$$
\operatorname{dim} \mathfrak{U}^{*} \leqq \operatorname{dim} U \leqq \prod_{1 \leqq i \leqq r}\left(\Lambda_{0}\left(H_{i}\right)+1\right) .
$$

The theorem is now proved completely.

Part II. Infinite-dimensional representations of complex semisimple Lie algebras. Let $R$ and $C$ be the fields of real and complex numbers respectively. Let $\Omega_{0}$ be a Lie algebra over $R$ with a semisimple subalgebra $\Omega_{K, 0}$ such that there exists a linear mapping $\Gamma$ of $\Omega_{K, 0}$ into $\ell_{0}$ with the following properties:

$$
\begin{aligned}
\mathfrak{l}_{0} & =\mathfrak{R}_{K, 0}+\Gamma\left(\mathfrak{R}_{K, 0}\right), \quad \mathfrak{l}_{K, 0} \cap \Gamma\left(\mathfrak{R}_{K, 0}\right)=\{0\}, \\
{\left[X_{1}, \Gamma\left(X_{2}\right)\right] } & =\Gamma\left(\left[X_{1}, X_{2}\right]\right), \quad\left[\Gamma\left(X_{1}\right), \Gamma\left(X_{2}\right)\right]=-\left[X_{1}, X_{2}\right]
\end{aligned}
$$

for any $X_{1}, X_{2} \in \mathbb{R}_{K, 0}$.

(11) This follows either from well known results on finite-dimensional representations of semisimple Lie algebras or from Lemma 11. 
Let $\mathbb{R}$ denote the complexification $\left({ }^{12}\right)$ of $\mathbb{R}_{0}$ and let $\mathbb{R}_{K}$ be the smallest subspace of $\mathbb{R}$ containing $\mathfrak{R}_{K, 0}$. Then clearly $\mathbb{R}_{K}$ is a Lie algebra which is the complexification of $\mathfrak{R}_{K, 0}$. We extend $\Gamma$ on $\mathfrak{R}_{K}$ by linearity and put( $\left.{ }^{13}\right)$

$\gamma(X)=\frac{1}{2}\left(X+(-1)^{1 / 2} \Gamma(X)\right), \quad \gamma^{+}(X)=\frac{1}{2}\left(X-(-1)^{1 / 2} \Gamma(X)\right) \quad\left(X \in \mathbb{R}_{K}\right)$.

Let $\mathfrak{g}=\boldsymbol{\gamma}\left(\mathfrak{R}_{K}\right), \mathfrak{g}^{+}=\boldsymbol{\gamma}^{+}\left(\mathfrak{R}_{K}\right)$. Then $\mathfrak{g}$ and $\mathfrak{g}^{+}$are ideals in $\mathfrak{R}$ and $\mathfrak{R}=\mathfrak{g}+\mathfrak{g}^{+}$, $\mathfrak{g} \cap \mathfrak{g}^{+}=\left[\mathfrak{g}, \mathfrak{g}^{+}\right]=\{0\}$. Also $\gamma$ and $\gamma^{+}$are isomorphisms of $\mathfrak{R}_{K}$ on $\mathfrak{g}$ and $\mathfrak{g}^{+}$respectively. Since $\mathfrak{R}_{K, 0}$ is semisimple, $\mathfrak{R}_{K}, \mathfrak{g}, \mathfrak{g}^{+}$, and $\mathfrak{R}$ are all semisimple.

Let $\mathfrak{B}$ denote the universal enveloping algebra $\left({ }^{9}\right)$ of $\mathfrak{R}$. Let $\mathfrak{A}, \mathfrak{X}$, and $\mathfrak{A}^{+}$ be the subalgebras of $\mathfrak{B}$ generated by the sets $(1, \mathfrak{g}),\left(1, \mathfrak{R}_{K}\right)$, and $\left(1, \mathfrak{g}^{+}\right)$respectively. We claim that $\mathfrak{A}, \mathfrak{X}$, and $\mathfrak{A}^{+}$are the universal enveloping algebras of $\mathfrak{g}, \mathfrak{R}_{K}$, and $\mathfrak{g}^{+}$respectively. This follows from the following lemma.

LEMMA 21. Let $\mathfrak{M}$ be a Lie algebra over a field $k$ of characteristic zero and let $\mathfrak{N}$ be a subalgebra of $\mathfrak{M}$. Let $\mathfrak{U}$ be the universal enveloping algebra of $\mathfrak{M}$ and $\mathfrak{B}$ the subalgebra of $\mathfrak{U}$ generated by $\mathfrak{N}$ and 1 . Then $\mathfrak{B}$ is isomorphic to the universal enveloping algebra of $\mathfrak{N}$.

Choose a base $x_{i}, 1 \leqq i \leqq m$, for $\mathfrak{M}$ such that $x_{i}, 1 \leqq i \leqq n, n \leqq m$, is a base for $\mathfrak{R}$. Let $\mathfrak{B}^{\prime}$ be the universal enveloping algebra of $\mathfrak{N}$. Then clearly there is a homomorphism $\varphi$ of $\mathfrak{B}^{\prime}$ onto $\mathfrak{B}$ such that $\varphi$ leaves every element of $\mathfrak{N}$ fixed and $\varphi\left(1^{\prime}\right)=1,1^{\prime}$ being the unit element of $\mathfrak{B}^{\prime}$. We define as in [9] the basic canonical elements in $\mathfrak{U}$ and $\mathfrak{B}^{\prime}$ with respect to the bases $x_{i}, 1 \leqq i \leqq m$, and $x_{i}$, $1 \leqq i \leqq n$, for $\mathfrak{M}$ and $\mathfrak{N}$ respectively. Then it is clear from their definition that if $z_{j}^{\prime}, 1 \leqq j \leqq r$, are any distinct basic canonical elements in $\mathfrak{B}^{\prime}, \varphi\left(\boldsymbol{z}_{j}^{\prime}\right), 1 \leqq j \leqq r$, are also distinct basic canonical elements in $\mathfrak{U}$. Now let $z^{\prime} \in \mathfrak{B}^{\prime}$. Since the basic canonical elements form a base for $\mathfrak{B}^{\prime}, \boldsymbol{z}^{\prime}=\sum_{1 \leqq i \leqq r} c_{\boldsymbol{i}} z_{i}^{\prime}$ where $c_{\boldsymbol{i}} \in k$ and $\boldsymbol{z}_{\boldsymbol{i}}^{\prime}$, $1 \leqq i \leqq r$, are distinct basic canonical elements in $\mathfrak{B}^{\prime}$. Hence $\varphi\left(z^{\prime}\right)=\sum_{1 \leqq i \leqq r}$ $c_{i} \varphi\left(z_{i}^{\prime}\right)$. Since distinct basic canonical elements are linearly independent in $\mathfrak{U}$ it follows that if $\varphi\left(z^{\prime}\right)=0, c_{i}=0,1 \leqq i \leqq r$, and therefore $z^{\prime}=0$. Hence $\varphi$ is an isomorphism.

We make the convention that whenever we speak of a representation of the universal enveloping algebra $\mathfrak{U}$ of a Lie algebra $\mathfrak{M}$ on a vector space $V$ it will always be assumed implicitly that $\pi(1)=I$ where $I$ is the identity mapping of $V$. Then it is clear that there is a 1-1 correspondence between representations of $\mathfrak{U}$ and those of $\mathfrak{M}$ such that corresponding representations coincide on $\mathfrak{M}$. We shall usually denote these corresponding representations by the same symbol. In particular if $\mathfrak{M}$ is semisimple every finite-dimensional representation of $\mathfrak{M}$ (and therefore of $\mathfrak{u}$ ) is fully reducible.

Choose a fixed Cartan subalgebra $\mathfrak{h}_{K}$ of $\mathfrak{R}_{K}$ and a fundamental system of roots $\left\{\alpha_{1}, \cdots, \alpha_{l}\right\}$ of $\mathfrak{R}_{K}$ with respect to $\mathfrak{h}_{K}$. Let $\mathrm{P}_{K}$ be the set of all dominant

(12) This means that $\mathfrak{l}$ is a Lie algebra obtained from $\mathfrak{l}_{0}$ by extending the ground field from $R$ to $C$.

(13) We fix once for all an element $(-1)^{1 / 2}$ in $C$ such that $\left((-1)^{1 / 2}\right)^{2}=-1$. 
integral linear functions on $\mathfrak{h}_{K}$. Then from Theorems 1 and 2 we know that there is a 1-1 correspondence between equivalence classes of finite-dimensional representations of $\mathfrak{R}_{K}$ (and therefore of $\mathfrak{X}$ ) and dominant integral functions $\Lambda$ on $\mathfrak{h}_{K}$ such that if $\mathfrak{D}_{\Lambda}$ is the class corresponding to $\Lambda$ then $\Lambda$ is the highest weight of any representation in $\mathfrak{D}_{\Lambda}$. Let $\pi \in \mathfrak{D}_{\Lambda}$. Then every weight of $\pi$ will be called a weight of $\mathfrak{D}_{\Lambda}$. In particular $\Lambda$ is the highest weight of $\mathfrak{D}_{\Delta}$.

Let $\pi$ be any representation $\mathfrak{X}$ on a vector space $V$ (not necessarily finitedimensional). For any fixed $\psi \in V$ put $U=\pi(\mathfrak{X}) \psi$. Let $\pi^{\prime}$ be the representation of $\mathfrak{X}$ induced on $U$. Given any $\Lambda \in \mathrm{P}_{K}$ we say that $\psi$ transforms under $\pi$ according to $\mathfrak{D}_{\Lambda}$ if either $\psi=0$ or $\pi^{\prime}$ is finite-dimensional (and therefore fully reducible) and every irreducible component of $\pi^{\prime}$ belongs to $\mathfrak{D}_{\Lambda}$. Let $V_{\Delta}$ be the set of all elements of $V$ which transform according to $\mathfrak{D}_{\Lambda}$. It is clear from its definition that $V_{\Delta}$ is an invariant subspace of $V$.

Lemma 22. The sum $\sum_{\Lambda \in \mathrm{P}_{K}} V_{\Delta}$ is direct and if $U$ is any invariant subspace of $V$ then

$$
U \cap\left(\sum_{\Lambda \in \mathbb{P}_{K}} V_{\Lambda}\right)=\sum_{\Lambda \in \mathbb{P}_{K}}\left(U \cap V_{\Lambda}\right) .
$$

The proof is similar to that of Lemma 1. Let $\psi \in U \cap\left(\sum_{\Lambda \in \mathrm{P}_{K}} V_{\Lambda}\right)$. Then $\psi=\psi_{1}+\cdots+\psi_{r}$ where $\psi_{i} \in V_{\Lambda_{i}}$ and $\Lambda_{i} \neq \Lambda_{j}, 1 \leqq i, j \leqq r(i \neq j)\left(\Lambda_{i} \in \mathrm{P}_{K}\right)$. Choose some representation $\pi_{\Lambda_{i}}$ of $\mathfrak{X}$ in $\mathfrak{D}_{\Lambda_{i}}, 1 \leqq i \leqq r$. Then $\pi_{\Lambda_{i}}$ are all inequivalent representations. Hence given any $j, 1 \leqq j \leqq r$, we can, from Lemma 4 of [9], find an element $x \in \mathfrak{X}$ such that $\pi_{\Lambda_{j}}(x)=\pi_{\Lambda_{j}}(1), \pi_{\Lambda_{i}}(x)=0 \quad(i \neq j)$. But then it is clear that $\pi(x) \psi=\psi_{j}$. Since $U$ is invariant under $\pi, \psi_{j}=\pi(x) \psi$ $\in U$. Hence $U \cap\left(\sum_{\Lambda \in \mathrm{P}_{K}} V_{\Lambda}\right)=\sum_{\Lambda \in \mathrm{P}_{K}}\left(U \cap V_{\Lambda}\right)$. Now, if we take $U=\{0\}$ we find that if $\psi=0$ then $\psi_{j}=0,1 \leqq j \leqq r$. Hence the sum $\sum_{\Lambda \in \mathrm{P}_{K}} V_{\Delta}$ is direct.

Since $V_{\Delta}$ is invariant under $\pi$ we can, for any linear function $\lambda$ on $\mathfrak{h}$, define $V_{\Delta, \lambda}$ just as in Lemma 1.

Lemma 23. $V_{\Delta}=\sum_{\lambda} V_{\Lambda, \lambda}$.

Let $\psi \in V_{\Delta}$. Put $U=\pi(\mathfrak{X}) \psi$. Then by the definition of $V_{\Delta}, U$ is finite-dimensional. But from the theory of finite-dimensional representations it is known that $U=\sum_{\lambda} U_{\lambda}$. Since clearly $U_{\lambda} \subset V_{\Lambda, \lambda}$, it follows that $\psi \in \sum_{\lambda} V_{\Lambda, \lambda}$. This being true for every $\psi \in V_{\Lambda}, V_{\Lambda}=\sum_{\lambda} V_{\Delta, \lambda}$. Notice that $V_{\Lambda, \lambda}=\{0\}$ if $\lambda$ is not a weight of $\mathfrak{D}_{\Lambda}$.

Let $\pi$ be any representation of $\mathfrak{B}$ on $V$. Then $\pi$ defines a representation of $\mathfrak{X}$ on $V$ and for each $\Lambda \in \mathrm{P}_{K}$ we can construct the subspace $V_{\Lambda}$ of Lemma 22 with respect to $\pi(\mathfrak{X})$. Given any $\Lambda_{0} \in P_{K}$ we say that $\mathfrak{D}_{\Lambda_{0}}$ occurs in $\pi$ if $V_{\Lambda_{0}}$ $\neq\{0\}$. Also we shall say that $\mathfrak{D}_{\Lambda_{0}}$ occurs a finite or an infinite number of times according as $\operatorname{dim} V_{\Lambda_{0}}$ is finite or infinite. Let $\mathfrak{S}$ be the center of $\mathfrak{A}$ and $\chi$ a homomorphic mapping of $\mathbb{E}$ into $C$. Our object is to prove the following theorem. 
THEOREM 4. Given $\Lambda_{0}$ and $\chi$ there exist only a finite number of inequivalent irreducible representations $\pi$ of $\mathfrak{B}$ such that $\mathfrak{D}_{\Lambda_{0}}$ occurs in $\pi$ and $\left({ }^{14}\right) \pi(z-\chi(z))=0$ for all $z \in \mathfrak{C}$. Further if $\pi$ is such a representation then $\mathfrak{D}_{\Delta}$ occurs only a finite number of times in $\pi$ for every $\Lambda \in \mathrm{P}_{K}$ and 8 being the center of $\mathfrak{B}$ we can find $a$ homomorphism $\xi$ of 8 into $C$ such that $\pi(z-\xi(z))=0$ for all $z \in B$.

First we need some lemmas. Since $g$ is isomorphic to $\mathfrak{\Omega}_{K}$ under the mapping $\gamma$ every representation of $\mathfrak{R}_{K}$ determines a representation of $g$ and conversely. Hence for any $\Lambda \in \mathrm{P}_{K}, \mathfrak{D}_{\Lambda}$ can also be regarded as a class of representations of g. If $\pi$ is any representation of $\mathfrak{A}$ on $V$ we can define exactly as before the subspace $V_{\Delta}$ of $V$ consisting of all elements which transform under $\pi(\mathfrak{A})$ according to $\mathfrak{D}_{\Lambda}$. In particular let $\pi$ be the adjoint representation of $\mathfrak{A}$ on itself given by

$$
\pi(Z) w=[Z, w] \quad(Z \in \mathfrak{g}, w \in \mathfrak{A}) .
$$

Then for every $\Lambda \in \mathrm{P}_{K}$ we can define $\mathfrak{A}_{\Lambda}$.

\section{Lemma 24. $\mathfrak{A}=\sum_{\Lambda \in \mathrm{P}_{K}} \mathfrak{A}_{\Lambda}$ and for every $\Lambda, \mathfrak{A}_{\Delta}$ is a finite module over $\mathfrak{E}$.}

We shall derive this lemma from a well known result in the theory of invariants. Let $\rho$ and $\sigma$ be any two matrix representations of $\mathfrak{g}$ of degree $p$ and $q$ respectively. Let $x_{i}, 1 \leqq i \leqq p$, and $y_{j}, 1 \leqq j \leqq q$, be two sets of independent indeterminates. Let $C[x, y]$ denote the (commutative) ring of all polynomials in $(x)$ and $(y)$ with coefficients in $C$. For every $Z \in \mathfrak{g}$ we define a $C$-derivation $\left({ }^{15}\right) D_{z}$ of $C[x, y]$ which is uniquely determined by the relations

$$
D_{Z} x_{j}=\sum_{1 \leqq i \leqq p} x_{i} \rho_{i j}(Z), \quad 1 \leqq j \leqq p ; \quad D_{Z} y_{j}=\sum_{1 \leqq i \leqq q} y_{i} \sigma_{i j}(Z), \quad 1 \leqq j \leqq q .
$$

The mapping $Z \rightarrow D_{Z}(Z \in \mathfrak{g})$ is easily seen to be a representation of $\mathfrak{g}$ on $C[x, y]$. An element $f \in C[x, y]$ is said to be an invariant if $D_{z} f=0$ for all $Z \in \mathfrak{g}$. Since $\mathrm{g}$ is semisimple the main theorem of the theory of invariants is applicable in this case (see Weyl $[13$, p. 274]). It may be stated as follows.

THEOREM. There exist a finite number of invariants, $J_{\nu}, 1 \leqq \nu \leqq N$, such that the ring $C\left[J_{1}, \cdots, J_{N}\right]$ contains all invariants.

We shall now use this theorem to prove Lemma 25. $f\left(i_{1}, \cdots, i_{r}\right)$ being any function of $r$ indices (all running from 1 to $n$ ) with values in a vector space over $C$, we denote by $S_{\left(i_{1}, \cdots, i_{r}\right)} f\left(i_{1}, \cdots, i_{r}\right) 1 / r$ ! times the sum of $f\left(j_{1}, \cdots, j_{r}\right)$ for all permutations $\left(j_{1}, \cdots, j_{r}\right)$ of $\left(i_{1}, \cdots, i_{r}\right)$. Such a function will be called symmetric if $f\left(i_{1}, \cdots, i_{r}\right)=S_{\left(i_{1}, \cdots, i_{r}\right)} f\left(i_{1}, \cdots, i_{r}\right)$ for all $1 \leqq i_{1}, \cdots$, $i_{r} \leqq n$.

Choose a base $Z_{i}, 1 \leqq i \leqq n$, for $\mathrm{g}$. Then $S_{\left(i_{1}, \cdots, i_{r}\right)} Z_{i_{1}} Z_{i_{2}} \cdots Z_{i_{r}}$ is a basic

(14) We shall assume throughout that $\chi(1)=1$. For the only other possibility is $\chi(1)=0$. But in this case $\pi(1)=0$ and so in accordance with our convention $\pi$ is not a representation.

(15) This means that $D_{Z}$ is a linear mapping of $C[x, y]$ such that $D_{Z}(f g)=\left(D_{Z} f\right) g+f\left(D_{Z} g\right)$ for any $f, g \in C[x, y]$. 
canonical element in $\mathfrak{A}$ with respect to this base. Let $V_{r}$ be the subspace of $\mathfrak{A}$ spanned by all such elements for a fixed value of $r \geqq 0$. Also put $V_{-1}=\{0\}$. Clearly $V_{r}$ is invariant under the adjoint representation $\pi$ of $\mathfrak{A}$. Since $V_{r}$ is finite-dimensional it is fully reducible under $\pi$ and therefore $V_{r} \subset \sum_{\Lambda} \mathfrak{A}_{\Lambda}$. But since the basic canonical elements span $\mathfrak{A}, \mathfrak{A}=\sum_{r \geq 0} V_{r} \subset \sum_{\Lambda} \mathfrak{A}_{\Lambda}$. Hence $\mathfrak{A}=\sum_{\Lambda} \mathfrak{A}_{\Lambda}$. But then from Lemma 22, $V_{r}=\sum_{\Lambda}\left(V_{r} \cap \mathfrak{A}_{\Lambda}\right)$. Hence

$$
\sum_{\Lambda} \mathfrak{A}_{\Lambda}=\mathfrak{A}=\sum_{r \geq 0} V_{r}=\sum_{r \geq 0} \sum_{\Lambda}\left(V_{r} \cap \mathfrak{A}_{\Lambda}\right)=\sum_{\Lambda} \sum_{r \geq 0}\left(V_{r} \cap \mathfrak{A}_{\Lambda}\right)=\sum_{\Lambda} \mathfrak{A}_{\Lambda}^{\prime}
$$

where $\mathfrak{H}_{\Lambda}^{\prime}=\sum_{r \geq 0}\left(V_{r} \cap \mathfrak{A}_{\Lambda}\right)$. Since $\mathfrak{A}_{\Lambda}^{\prime} \subset \mathfrak{A}_{\Lambda}$ and since, by Lemma 22 , the sum $\sum_{\Lambda} \mathfrak{A}_{\Lambda}$ is direct it follows that $\mathfrak{H}_{\Lambda}^{\prime}=\mathfrak{A}_{\Lambda}$. Hence

$$
\mathfrak{A}_{\Lambda}=\sum_{r \geqq 0}\left(V_{r} \cap \mathfrak{A}_{\Lambda}\right) .
$$

The sum on the right-hand side is direct since distinct basic canonical elements are linearly independent.

Now let $\rho$ be the adjoint representation of $g$ and $\sigma^{*}$ any irreducible representation of $\mathfrak{g}$ on a vector space $U^{*}$ such that $\sigma^{*} \in \mathfrak{D}_{\Lambda}$. Let $U$ be the space dual to $U^{*}$ and $\sigma$ the representation of $\mathfrak{g}$ induced on $U$. Let $e_{i}, 1 \leqq i \leqq q$, be a base for $U$. Then $\sigma$ can be regarded as a matrix representation with respect to this base, so that

$$
\sigma(Z) e_{j}=\sum_{1 \leqq i \leqq q} e_{i} \sigma_{i j}(Z) \quad(Z \in \mathfrak{g}, 1 \leqq j \leqq q) .
$$

Similarly

$$
\rho(Z) Z_{j}=\left[Z, Z_{j}\right]=\sum_{1 \leqq i \leqq n} Z_{i} \rho_{i j}(Z) \quad(Z \in \mathfrak{g}, 1 \leqq j \leqq n) .
$$

Now we apply the above theorem to the pair $(\rho, \sigma)$. It is clear that if a polynomial $f \in C[x, y]$ is an invariant then all the doubly homogeneous components $\left({ }^{16}\right)$ of $f$ are also invariants. Hence we may assume that $J_{1}, \cdots, J_{N}$ are all themselves doubly homogeneous. Among these let $G_{1}(x), \cdots, G_{m}(x)$ be all those which are independent of $(y)$ and $H_{\nu}(x, y), 1 \leqq \nu \leqq s$, all those which are linear in $(y)$. Then it is evident that any invariant $f(x, y)$ which is homogeneous and linear in $(y)$ must belong to $\sum_{1 \leqq v \leqq s} \Omega H_{\nu}(x, y)$ where $\Omega$ $=C\left[G_{1}, \cdots, G_{m}\right]$. Let $r_{\nu}$ be the degree of $H_{\nu}(x, y)$ in $x$. Then



where $h_{\nu}^{i_{1} 1_{2} \ldots i_{r}, j} \in C$ and we may assume that $h_{\nu}^{i_{1} t_{2} \ldots i_{r}, j}$ are symmetric with respect to $i_{1}, \cdots, i_{r_{v}}$. Put

(16) By doubly homogeneous we mean homogeneous in each of the two sets of variables $(x)$ and $(y)$ separately. 


$$
H_{\nu}^{j}(Z)=\sum_{1 \leqq i_{1}, \cdots, i_{r \nu} \leqq m} h_{\nu}^{i_{1} i_{2} \cdots i_{r \nu}, j} Z_{i_{1}} Z_{i_{2}} \cdots Z_{i_{r \nu}}, \quad 1 \leqq j \leqq q .
$$

We shall prove that $H_{\nu}^{j}(Z), 1 \leqq \nu \leqq s, 1 \leqq j \leqq q$, form a $\left(5\right.$-basis for $\mathfrak{A}_{\Delta}$. Consider the element $\sum_{1 \leqq j \leqq q} H_{\nu}^{j}(Z) \times e_{j}$. If we extend $\rho$ to a representation of $\mathfrak{g}$ on $\mathfrak{A}$ by setting $\rho(Z) w=[Z, w](Z \in \mathfrak{g}, w \in \mathfrak{U})$ we get the representation $\rho+\sigma$ of $\mathfrak{g}$ on the Kronecker product $\mathfrak{A} \times U$. Since $H_{\nu}(x, y)$ is an invariant it is clear that $\sum_{1 \leqq j \leqq q} H_{\nu}^{j}(Z) \times e_{j}$ is invariant under $\rho+\sigma$. Therefore it follows that the space $W$ spanned by $H_{\nu}^{j}(Z), 1 \leqq j \leqq q$, is invariant under $\rho$ and the representation induced on it by $\rho$ must be dual to $\sigma$ unless $W=\{0\}$. Hence in any case $H_{\nu}^{j}(Z) \in \mathfrak{A}_{\Lambda}, 1 \leqq j \leqq q$. Therefore in order to show that

$$
\mathfrak{A}_{\Lambda}=\sum_{j, \nu} \mathfrak{S} H_{\nu}^{j}(Z)
$$

it is sufficient to prove that

$$
\mathfrak{A}_{\Lambda} \cap V_{r} \subset \sum_{j, \nu} \mathfrak{S} H_{\nu}^{j}(Z), \quad r \geqq-1,
$$

since $\mathfrak{A}_{\Lambda}=\sum_{r \geqq 0}\left(\mathfrak{A}_{\Lambda} \cap V_{r}\right)$. We shall prove this by induction on $r$. For $r=-1$ this is true trivially. Hence suppose $r \geqq 0$. Let $z \in \mathfrak{A}_{\Lambda} \cap V_{r}$. We have to prove that $z \in \sum_{j, \nu} \subseteq H_{\nu}^{j}(Z)$. Since $\mathfrak{A}_{\Lambda} \cap V_{r}$ is finite-dimensional it is completely reducible under $\rho$. Hence it is sufficient to consider the case when $z \neq 0$ and $W=\pi(\mathfrak{A}) z$ is irreducible under $\pi$. ( $\pi$ is the adjoint representation of $\mathfrak{A}$. Since $z$ transforms according to $\mathfrak{D}_{\Delta}$ we can choose a base $w_{j}, 1 \leqq j \leqq q$, such that $\sum_{1 \leqq j \leqq q} w_{j} \times e_{j}$ is invariant under $\rho+\sigma$. Let

$$
w_{j}=\sum_{1 \leqq i_{1}, \cdots, i_{r} \leqq n} a^{i_{1} i_{2} \cdots i_{r}, j} Z_{i_{1}} Z_{i_{2}} \cdots Z_{i_{r}}, \quad 1 \leqq j \leqq q,
$$

where the coefficients $a^{i_{1} i_{2} \cdots i_{r}, j}$ are symmetric in $i_{1}, \cdots, i_{r}$. Then it is easily verified that the form

$$
f(x, y)=\sum_{1 \leqq i_{1}, \cdots, i_{r} \leqq n, 1 \leqq j \leqq q} a^{i_{1} i_{2} \cdots i_{r}, j} x_{i_{1} x_{i_{2}}} \cdots x_{i_{r}} y_{j}
$$

is invariant with respect to $(\rho, \sigma)$. Hence

$$
f(x, y)=\sum_{1 \leqq \nu \leqq s} M_{\nu}(x) H_{\nu}(x, y)
$$

where $M_{\nu}(x)$ is either zero or an invariant form of degree $r-r_{\nu}$. We may assume that $M_{\nu}(x) \neq 0$ for $1 \leqq \nu \leqq s^{\prime}$ and $M_{\nu}(x)=0$ for $\nu>s^{\prime}$. Then if $s_{\nu}=r-r_{\nu}$,

$$
M_{\nu}(x)=\sum_{1 \leqq i_{1}, \cdots, i_{\nu} \leqq n} b_{\nu}^{i_{1} i_{2} \cdots i_{s_{\nu}}} x_{i_{1}} x_{i_{2}} \cdots x_{i_{\nu}}, \quad 1 \leqq \nu \leqq s^{\prime},
$$


where the coefficients are again symmetric with respect to $i_{1}, i_{2}, \cdots, i_{s_{\nu}}$. Hence comparing coefficients we have

$$
a^{i_{1} i_{2} \cdots i_{r}, j}=\sum_{1 \leqq \nu \leqq 8^{\prime}} S\left(_{\left(i_{1}, \cdots, i_{r}\right)}\left(b_{\nu}^{i_{1} i_{2} \cdots i_{s_{\nu}}} h_{\nu}^{i_{\delta_{\nu}+1} \cdots i_{r}, j}\right) .\right.
$$

Therefore

$$
\begin{aligned}
w_{j} & =\sum_{1 \leqq i_{1}, \cdots, i_{r} \leqq n} a^{i_{1} i_{2} \cdots i_{r}, j} Z_{i_{1} Z_{i_{2}}} \cdots Z_{i_{r}} \\
& =\sum_{1 \leqq \nu \leqq 8^{\prime}} \sum_{1 \leqq i_{1}, \cdots, i_{r} \leqq n} b_{\nu}^{i_{1} i_{2} \cdots i_{s_{\nu}}} h_{\nu}^{i_{s_{\nu}+1} \cdots i_{r}, j} \underbrace{S}_{\left(i_{1}, \cdots, i_{r}\right)} Z_{i_{i}} Z_{i_{2}} \cdots Z_{i} \\
& \equiv \sum_{1 \leqq \nu \leqq 8^{\prime}} \sum_{1 \leqq i_{1}, \cdots, i_{r} \leqq n} b_{\nu}^{i_{1} i_{2} \cdots i_{s_{\nu}}} h_{\nu}^{i_{s_{\nu}+1} \cdots i_{r}, j} Z_{i_{1}} Z_{i_{2}} \cdots Z_{i_{r}} \bmod \sum_{-1 \leqq p<r} V_{p},
\end{aligned}
$$

since

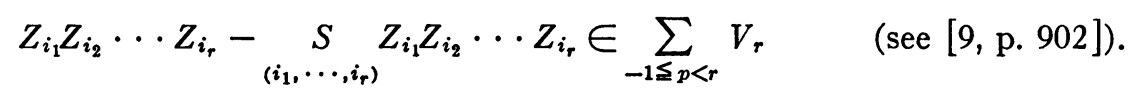

Also

$$
\sum_{1 \leqq} \sum_{1 \leqq i_{1}, \cdots, i_{r} \leqq n} b_{\nu}^{i_{1} i_{2} \cdots i_{\delta_{\nu}}} h_{\nu}^{i_{\delta_{\nu}+1} \cdots i_{r}, j} Z_{i_{1}} Z_{i_{2}} \cdots Z_{i_{r}}=\sum_{1 \leqq \nu \leqq{\delta^{\prime}}^{\prime}} \omega_{\nu} H_{\nu}^{j}(Z)
$$

where

$$
\omega_{\nu}=\sum_{1 \leqq i_{1}, \cdots, i_{\ell_{\nu}} \leqq n} b_{\nu}^{i_{1} \cdots i_{s_{\nu}}} Z_{i_{1}} Z_{i_{2}} \cdots Z_{i_{s_{\nu}}} \in \mathcal{E}
$$

since $M_{\nu}(x)$ is an invariant. Since $w_{j}, H_{\nu}^{j}(Z) \in \mathfrak{A}_{\Lambda}$ and $\omega_{\nu} \in \mathbb{S}$ it is clear that $w_{j}-\sum_{1 \leqq \nu \leqq s} \omega_{\nu} H_{\nu}^{j}(Z) \in \mathfrak{A}_{\Delta}$. Therefore

$$
w_{j}-\sum_{1 \leqq \nu \leqq g^{\prime}} \omega_{\nu} H_{\nu}^{j}(Z) \in \mathfrak{H}_{\Lambda} \cap\left(\sum_{-1 \leqq p<r} V_{p}\right)=\sum_{-1 \leqq p<r}\left(\mathfrak{A}_{\Lambda} \cap V_{p}\right)
$$

since $\mathfrak{A}_{\Lambda}=\sum_{p \leqq 0}\left(V_{p} \cap \mathfrak{A}_{\Lambda}\right)$. Hence by induction hypothesis

$$
w_{j}-\sum_{1 \leqq \nu \S_{8^{\prime}}} \omega_{\nu} H_{\nu}^{j}(Z) \in \sum_{1 \leqq j \leqq q, 1 \leqq \nu \leqq s}\left(5 H_{\nu}^{j}(Z) .\right.
$$

Therefore

$$
w_{j} \in \sum_{1 \leqq j \leqq q, 1 \leqq \nu \leqq s} \subseteq H_{\nu}^{j}(Z)
$$

and the lemma is proved.

For a given $\Lambda_{0} \in P_{K}$ let $\pi$ be any irreducible representation of $\mathfrak{X}$ on a space $V$ such that $\pi \in \mathfrak{D}_{\Lambda_{0}}$. Choose an element $\psi \in V(\psi \neq 0)$ which belongs to the highest weight $\Lambda_{0}$. Since from Theorem 2 the multiplicity of $\Lambda_{0}$ in $\pi$ is $1, \psi$ 
is unique apart from a numerical factor. Let $\mathfrak{Y}_{\Lambda_{0}}$ be the left ideal in $\mathfrak{X}$ consisting of all elements $x \in \mathfrak{X}$ such that $\pi(x) \psi=0$. Let $z \rightarrow z^{*}$ denote the natural mapping of $\mathfrak{X}$ on $\mathfrak{X}^{*}=\mathfrak{X} / \mathfrak{Y}_{\Lambda_{0}}$ and $\pi^{*}$ the natural representation of $\mathfrak{X}$ on $\mathfrak{X}^{*}$. Since $V$ is irreducible $\mathfrak{Y}_{\Lambda_{0}}$ is a maximal left ideal and it is easily seen that $\pi$ is equivalent to $\pi^{*}$ under the isomorphism $\pi(z) \psi \leftrightarrow z^{*}(z \in \mathfrak{X})$ of $V$ with $\mathfrak{X}^{*}$. Further $\mathfrak{Y}_{\Lambda_{0}}$ is uniquely determined by $\Lambda_{0}$ since the vector $\psi$ is essentially unique. We now define a representation $\rho^{*}$ of $\mathfrak{R}$ on $\mathfrak{X}^{*}$ by

$$
\rho^{*}(X)=\pi^{*}(X), \quad X \in \Omega_{K} ; \quad \rho^{*}(Z)=0, \quad Z \in g .
$$

It is easily checked that $\rho^{*}$ is a representation. Let $\sigma$ denote the representation of $\mathfrak{R}$ on $\mathfrak{A}$ given as follows:

$$
\begin{aligned}
\sigma(X) z & =[X, z] & \left(X \in \mathfrak{R}_{K}, z \in \mathfrak{A}\right), \\
\sigma(Z) z & =Z z & (Z \in \mathfrak{g}, z \in \mathfrak{A}) .
\end{aligned}
$$

It is again easy to verify that this is indeed a representation. Let $\nu$ be the uniquely determined representation of $\mathfrak{B}$ on the Kronecker product $\mathfrak{A} \times \mathfrak{X}^{*}$ which coincides with $\sigma+\rho^{*}$ on $\mathfrak{R}$. For any $\Lambda \in \mathrm{P}_{K}$ we consider the subspace $\left(\mathfrak{A} \times \mathfrak{X}^{*}\right)_{\Lambda}$ consisting of all elements of $\mathfrak{A} X \mathfrak{X}^{*}$ which transform under $\nu(\mathfrak{X})$ according to $\mathfrak{D}_{\Lambda}$.

Lemma 26. $\mathfrak{A} X \mathfrak{X}^{*}=\sum_{\Lambda \in \mathbb{P}_{K}}\left(\mathfrak{A} \times \mathfrak{X}^{*}\right)_{\Lambda}$ and $\left(\mathfrak{H} \times \mathfrak{X}^{*}\right)_{\Lambda}$ is a finite $(\mathfrak{S}$-module for each $\Lambda \in \mathrm{P}_{K}$.

Let $\pi$ denote the adjoint representation of $\mathfrak{A}$, and $\gamma$ the isomorphism of $\mathfrak{R}_{K}$ with $\mathfrak{g}$ as defined on p. 53. Since $[X, z]=[\gamma(X), z]\left(X \in \mathfrak{R}_{K}, z \in \mathfrak{A}\right)$ it follows that $\sigma(X)=\pi(\gamma(X)) \quad\left(X \in \mathfrak{R}_{K}\right)$. Hence for any $\Lambda \in \mathrm{P}_{K}$ the subspace $\mathfrak{A}_{\Lambda}$ consisting of all elements in $\mathfrak{A}$ which transform under $\sigma\left(\mathfrak{R}_{K}\right)$ according to $\mathfrak{D}_{\Lambda}$ is the same as $\mathfrak{A}_{\Lambda}$ of Lemma 25. Therefore we can find $a_{1}, \cdots, a_{r} \in \mathfrak{A}_{\Lambda}$ such that $\mathfrak{A}_{\Lambda}=\sum_{1 \leqq i \leqq r} \mathfrak{E} a_{i}$. Since $a_{i} \in \mathfrak{U}_{\Lambda}$ the space $\sigma(\mathfrak{X}) a_{i}=\pi(\mathfrak{A}) a_{i}$ is finitedimensional. Since $\mathfrak{X}^{*}$ is also finite-dimensional, the same holds for the space $\sigma(\mathfrak{X}) a_{i} \times \mathfrak{X}^{*}$ which is invariant under $\nu(\mathfrak{X})$. Hence it is completely reducible under $\nu(\mathfrak{X})$ and therefore

$$
a_{i} \times \mathfrak{X}^{*} \subset \sum_{\Lambda^{\prime} \in \mathrm{P}_{K}}\left(\mathfrak{I} \times \mathfrak{X}^{*}\right)_{\Lambda^{\prime}} .
$$

Now $\nu(\mathfrak{C})\left(a_{i} \mathbf{X X}^{*}\right)=\left(\mathfrak{C} a_{i} \mathbf{X}^{*}\right.$ and clearly each $\left(\mathfrak{A} \times \mathfrak{X}^{*}\right)_{\Lambda^{\prime}}$ is invariant under $\nu(\mathbb{E})$. Hence

$$
\mathfrak{A}_{\Lambda} \times \mathfrak{X}^{*} \subset \sum_{\Lambda^{\prime} \in \mathbf{P}}\left(\mathfrak{I} \times \mathfrak{X}^{*}\right)_{\Lambda^{\prime}}
$$

Since $\mathfrak{A}=\sum_{\Lambda} \mathfrak{A}_{\Lambda}$ from Lemma 25 , we get

$$
\mathfrak{A} \times \mathfrak{X}^{*}=\sum_{\Lambda}\left(\mathfrak{A}_{\Lambda} \times \mathfrak{X}^{*}\right)=\sum_{\Lambda \in \mathbf{P}_{K}}\left(\mathfrak{A} \times \mathfrak{X}^{*}\right)_{\Lambda} ;
$$

this proves the first statement in the lemma. 
Now we come to the second part. For any fixed $\Lambda \in \mathrm{P}_{K}$ choose an irreducible representation $\theta^{*}$ of $\Omega_{K}$ on a space $U^{*}$ such that $\theta^{*} \in \mathfrak{D}_{\Lambda}$. We extend $\theta^{*}$ to a representation of $\mathfrak{R}$ by defining $\theta^{*}(Z)=0(Z \in \mathfrak{g})$. Let $U$ be the space dual to $U^{*}$, and $\theta$ the representation of $\&$ induced on $U$. Let $\varphi$ denote the representation of $\mathfrak{B}$ on $\mathfrak{A} \times \mathfrak{X} \times U$ which coincides with $\nu+\theta$ on $\mathfrak{R}$. Given any $\Lambda^{\prime} \in \mathbf{P}_{K}$ choose $a_{i} \in \mathfrak{A}_{\Lambda^{\prime}}, 1 \leqq i \leqq r$, such that $\mathfrak{A}_{\Lambda^{\prime}}=\sum_{1 \leqq i \leqq r}\left(\mathfrak{S} a_{i}\right.$. Then $\sigma(\mathfrak{X}) a_{i}$ $X^{*} \times U$ is finite-dimensional and invariant under $\varphi(\mathfrak{X})$. Hence

$$
\sigma(\mathfrak{X}) a_{i} \times \mathfrak{X}^{*} \times U \subset \sum_{\Lambda^{\prime \prime} \in \mathrm{P}_{\mathbf{K}}}\left(\sigma(\mathfrak{X}) a_{i} \times \mathfrak{X}^{*} \times U\right)_{\Lambda^{\prime \prime}}
$$

where $\left(\sigma(\mathfrak{X}) a_{i} \times \mathfrak{X}^{*} \times U\right)_{\Lambda^{\prime \prime}}$ has the usual meaning. From this it follows as above that

$$
\mathfrak{H} \times \mathfrak{X}^{*} \times U=\sum_{\Lambda^{\prime} \in \mathbf{P}_{\mathbf{X}}}\left(\mathfrak{H} \times \mathfrak{X}^{*} \times U\right)_{\Lambda^{\prime}} .
$$

We now claim that $\left(\mathfrak{A} \mathbf{X} \mathfrak{X}^{*} \times \mathbf{X}\right)_{0}$ is a finite $\left(\mathfrak{S}\right.$-module. Since $\mathfrak{X}^{*} \times U$ is a finitedimensional space it is completely reducible under $\left(\rho^{*}+\theta\right)\left(\Omega_{K}\right)$. Let $\mathfrak{X}^{*} \times U$ $=\sum_{1 \leqq j \leqq N} U_{j}$ where the sum is direct and the subspaces $U_{j}$ are invariant and irreducible under $\left(\rho^{*}+\theta\right)\left(\Omega_{K}\right)$. Let $\Lambda_{j}$ be the highest weight of the representation of $\mathfrak{R}_{K}$ induced on $U_{j}^{*}$ where $U_{j}^{*}$ is the space dual to $U_{j}$. Then it is well known that $\mathfrak{D}_{0}$ occurs in the representation of $\mathfrak{R}_{K}$ induced on $\mathfrak{A}_{\Lambda^{\prime}} \times U_{j}$ if and only if $\Lambda^{\prime}=\Lambda_{j}$. Hence if $\Lambda^{\prime} \neq \Lambda_{j}$, it follows from Lemma 22 that

$$
\mathfrak{A}_{\Lambda^{\prime}} \times U_{j} \subset \sum_{\Lambda^{\prime \prime} \neq 0}\left(\mathfrak{A} \times \mathfrak{X}^{*} \times U\right)_{\Lambda^{\prime \prime}}
$$

Now

$$
\begin{aligned}
\mathfrak{A} \times \mathfrak{X}^{*} \times U & =\sum_{\Lambda, j}\left(\mathfrak{A}_{\Lambda} \times U_{j}\right)=\sum_{j}\left(\mathfrak{A}_{\Lambda_{j}} \times U_{j}\right)+\sum_{j} \sum_{\Delta \neq \Lambda_{j}}\left(\mathfrak{A}_{\Lambda} \times U_{j}\right) \\
& \subset \sum_{j}\left(\mathfrak{A}_{\Lambda_{j}} \times U_{j}\right)+\sum_{\Delta \neq 0}\left(\mathfrak{A} \times \mathfrak{X}^{*} \times U\right)_{\Lambda} .
\end{aligned}
$$

But from Lemma 22,

$$
\mathfrak{A}_{\Lambda_{j}} \times U_{j}=\sum_{\Lambda}\left\{\left(\mathfrak{A}_{\Lambda_{j}} \times U_{j}\right) \cap\left(\mathfrak{A} \times \mathfrak{X}^{*} \times U\right)_{\Lambda}\right\} .
$$

Hence

$$
\begin{aligned}
\mathfrak{A} \times \mathfrak{X}^{*} \times U \subset & \sum_{j}\left\{\left(\mathfrak{A}_{\Lambda_{j}} \times U_{j}\right) \cap\left(\mathfrak{A} \times \mathfrak{X}^{*} \times U\right)_{0}\right\} \\
& +\sum_{\Lambda \neq 0}\left(\mathfrak{A} \times \mathfrak{X}^{*} \times U\right)_{\Lambda} .
\end{aligned}
$$

Since the sum $\sum_{\Lambda}\left(\mathfrak{A} \times \mathfrak{X}^{*} \times U\right)_{\Lambda}$ is direct it follows that

$$
\left(\mathfrak{I} \times \mathfrak{X}^{*} \times U\right)_{0} \subset \sum_{j}\left\{\left(\mathfrak{A}_{\Lambda_{j}} \times U_{j}\right) \cap\left(\mathfrak{A} \times \mathfrak{X}^{*} \times U\right)_{0}\right\} \text {. }
$$


Hence

$$
\left(\mathfrak{A} \times \mathfrak{X}^{*} \times U\right)_{0}=\sum_{j}\left(\mathfrak{A}_{\Lambda} \times U_{j}\right)_{0} .
$$

Thus it is sufficient to prove that for a fixed $j,\left(\mathfrak{A}_{\Lambda_{j}} \times U_{j}\right)_{0}$ is a finite $(\mathfrak{S}$-module. Choose $a_{i} \in \mathfrak{A}_{\Lambda_{j}}, 1 \leqq i \leqq r$, such that $\mathfrak{A}_{\Lambda_{j}}=\sum_{1 \leqq i \leqq r} \Subset a_{i}$. Since $\sigma(\mathfrak{X}) a_{i} \times U_{j}$ is finite-dimensional, it is completely reducible under $\nu(\mathfrak{X})$. Hence

$$
\sigma(\mathfrak{X}) a_{i} \times U_{j}=\sum_{\mathbf{\Lambda}^{\prime}}\left(\sigma(\mathfrak{X}) a_{i} \times U_{j}\right)_{\mathbf{A}^{\prime}}
$$

Clearly $\nu(\mathfrak{C})\left(\sigma(\mathfrak{X}) a_{i} \times U_{j}\right)_{\Lambda^{\prime}} \subset\left(\mathfrak{A}_{\Lambda^{\prime}} \times U_{j}\right)_{\Lambda^{\prime}}$. But

$$
\mathfrak{A}_{\Lambda_{j}} \times U_{i}=\sum_{1 \leqq i \leqq r} \nu(\mathfrak{E})\left(\sigma(\mathfrak{X}) a_{i} \times U_{j}\right) .
$$

Therefore

$$
\left(\mathfrak{A}_{\Lambda_{j}} \times U_{j}\right)_{\Lambda^{\prime}}=\sum_{1 \leqq i \leqq r} \nu(\mathfrak{E})\left(\sigma(\mathfrak{X}) a_{i} \times U_{j}\right)_{\Lambda^{\prime}} .
$$

Since $\operatorname{dim}\left(\sigma(\mathfrak{X}) a_{i} \times U_{j}\right)_{\Lambda^{\prime}}$ is finite it follows that $\left(\mathfrak{A}_{\Lambda_{j}} \times U_{j}\right)_{\Lambda^{\prime}}$ is a finite $\mathfrak{E}$ module. If we take $\Lambda^{\prime}=0$ we get the required result.

Let $e_{j}, 1 \leqq j \leqq p$, be a base for $U$ and let $u_{i}, 1 \leqq i \leqq r$, be elements in $\left(\mathfrak{A} \times \mathfrak{X}^{*} \times U\right)_{0}$ such that

$$
\left(\mathfrak{A} \times \mathfrak{X}^{*} \times U\right)_{0}=\sum_{1 \leqq i \leqq r} \nu(\mathfrak{C}) u_{i} .
$$

Let $u_{i}=\sum_{1 \leqq j \leqq p} a_{i}^{j} \times e_{j}, 1 \leqq i \leqq r,\left(a_{i}^{j} \in \mathfrak{A} \times \mathfrak{X}^{*}\right)$. We claim that

$$
\left(\mathfrak{A} \times \mathfrak{X}^{*}\right)_{\Lambda}=\sum_{1 \leqq i \leqq r, 1 \leqq j \leqq p} \nu(\mathfrak{S}) a_{i}^{i} .
$$

Put $A=\sum_{1 \leqq i \leqq r, 1 \leqq j \leqq p} \nu(\mathbb{C}) a_{i}^{j}$. Since $u_{i}$ transform according to $\mathfrak{D}_{0}$ under $(\nu+\theta)\left(\Omega_{K}\right)$ it is clear that $a_{i}^{j} \in\left(\mathfrak{H} \times \mathfrak{X}^{*}\right)_{\Lambda}$. Hence $A \subset\left(\mathfrak{Y} \times \mathfrak{X}^{*}\right)_{\Lambda}$. Now let $a \in\left(\mathfrak{U} X \mathfrak{X}^{*}\right)_{\Delta}$. We have to show that $a \in A$. We may assume that $a \neq 0$. Then $\nu(\mathfrak{X}) a$ is a finite-dimensional space which is completely reducible under $\nu(\mathfrak{X})$ into a direct sum of invariant irreducible subspaces $V_{k}, 1 \leqq k \leqq q$. It is sufficient to show that $V_{k} \subset A, 1 \leqq k \leqq q$. For a fixed $k$ write $V=V_{k}$. Let $\psi$ be the irreducible representation of $\mathfrak{X}$ induced on $V$. Then $\psi \in \mathfrak{D}_{\Delta}$ and therefore we can choose a base $v_{j}, 1 \leqq j \leqq p$, for $V$ such that

$$
\sum_{1 \leqq j \leqq p}\left(v_{j} \times e_{j}\right) \in\left(\mathfrak{I} \times \mathfrak{X}^{*} \times U\right)_{0} .
$$

Hence

$$
\sum_{1 \leqq j \leqq p} v_{j} \times e_{j}=\sum_{1 \leqq i \leqq r} \nu\left(z_{i}\right) u_{i}=\sum_{1 \leqq i \leqq r, 1 \leqq j \leqq p} z_{i} a_{i}^{j} \times e_{j} \quad\left(z_{i} \in \mathfrak{E}\right) .
$$


Therefore $v_{j}=\sum_{1 \leqq i \leqq r} z_{i} a_{i}^{j} \in A, 1 \leqq j \leqq p$. This shows that $V \subset A$ and so the lemma is proved.

LEMMA 27. Let $\nu$ be the representation of $\mathfrak{B}$ on $\mathfrak{A} \times \mathfrak{X}^{*}$ as defined above. Then $\left({ }^{17}\right) \nu(\mathfrak{B})\left(1 \times 1^{*}\right)=\mathfrak{A} \times \mathfrak{X}^{*}$ and the set of all elements $b \in \mathfrak{B}$ such that $\nu(b)\{1 \times 1 *\}=0$ coincides with $\mathfrak{H Y}_{\Lambda_{0}}$.

If $a \in \mathfrak{A}$ and $x \in \mathfrak{X}$, it is easily seen that $\nu(a x)\left\{1 \times 1^{*}\right\}=a \times x^{*}$. Hence $\nu(\mathfrak{B})\left(1 \times 1^{*}\right)=\mathfrak{A} \times \mathfrak{X}^{*}$. Also since $[X, a] \in \mathfrak{A}$ for any $X \in \mathfrak{R}_{K}$ and $a \in \mathfrak{A}$ it is obvious that $\mathfrak{B}=\mathfrak{A} \mathfrak{X}$. Let $\mathfrak{M}$ be the set of all elements $b \in \mathfrak{B}$ such that $\nu(b)\left(1 \times 1^{*}\right)=0$. Clearly $\mathfrak{M}$ is a left ideal and $\mathfrak{M} \supset \mathfrak{Y}_{\Lambda_{0}}$. Hence $\mathfrak{M} \supset \mathfrak{H} \mathfrak{Y}_{\Lambda_{0}}$. On the other hand we can choose elements $\omega_{i}, 1 \leqq i \leqq N$, in $\mathfrak{X}$ such that $\omega_{i}^{*}$, $1 \leqq i \leqq N$, form a base for $\mathfrak{X}^{*}$. Since $\mathfrak{B}=\mathfrak{A X}$ every element $b \in \mathfrak{B}$ can be written as $b \equiv \sum_{1 \leqq i \leqq N} a_{i} \omega_{i} \bmod \mathfrak{A Y}_{\Lambda_{0}}\left(a_{i} \in \mathfrak{U}\right)$. Therefore

$$
\nu(b)\left(1 \times 1^{*}\right)=\sum_{1 \leqq i \leqq N} a_{i} \times \omega_{i}^{*} .
$$

Hence if $b \in \mathfrak{M}, a_{i}=0,1 \leqq i \leqq N$, and $b \in \mathfrak{Y} \mathfrak{Y}_{\Lambda_{0}}$. Therefore $\mathfrak{M}=\mathfrak{Y} \mathfrak{Y}_{\Lambda_{0}}$.

From Lemma 26, we can find elements $a_{i}, 1 \leqq i \leqq r$, in $\left(\mathfrak{A} \times \mathfrak{X}^{*}\right)_{\Lambda_{0}}$ such that $\left(\mathfrak{A} X \mathfrak{X}^{*}\right)_{\Lambda_{0}}=\sum_{1 \leqq i \leqq r} \nu(\mathfrak{E}) a_{i}$. Let $\nu_{0}$ be the representation of $\mathfrak{X}$ induced on $\left(\mathfrak{A} X \mathfrak{X}^{*}\right)_{\Lambda_{0}}$ by $\nu$. By Lemma 23 , we can write $\left(\mathfrak{H} X \mathfrak{X}^{*}\right)_{\Lambda_{0}}=\sum_{\lambda}\left(\mathfrak{I} X \mathfrak{X}^{*}\right)_{\Lambda_{0}, \lambda}$ where $\lambda$ runs over all the weights of $\nu_{0}$. Let $\nu_{0}(\mathfrak{X}) a_{i}=\sum_{\lambda}\left(\nu_{0}(\mathfrak{X}) a_{i}\right)_{\lambda}$ be the corresponding decomposition of the finite-dimensional space $\nu_{0}(\mathfrak{X}) a_{i}$. It is clear that $\nu(\mathfrak{C})\left(\nu_{0}(\mathfrak{X}) a_{i}\right)_{\lambda} \subset\left(\mathfrak{Y} \times \mathfrak{X}^{*}\right)_{\Lambda_{0}, \lambda}$. Hence

$$
\left(\mathfrak{A} \times \mathfrak{X}^{*}\right)_{\Lambda_{0}, \lambda}=\sum_{1 \leqq i \leqq r} \nu(\mathfrak{S})\left(\nu_{0}(\mathfrak{X}) a_{i}\right)_{\lambda} .
$$

Since $\nu_{0}(\mathfrak{X}) a_{i}$ is finite-dimensional this shows that each $\left(\mathfrak{A} \mathbf{X} \mathfrak{X}^{*}\right)_{\Lambda_{0}, \mathrm{\lambda}}$ is a finite (E-module. In particular this holds for $A=\left(\mathfrak{H} X \mathfrak{X}^{*}\right)_{\Lambda_{0}, \Lambda_{0}}$. Now we turn $A$ into an associative algebra as follows. For any $u \in A$ consider the finite-dimensional space $\nu_{0}(\mathfrak{X}) u$. It follows from the definition of $\left(\mathfrak{Y} X \mathfrak{X}^{*}\right)_{\Lambda_{0}}$ that the representation of $\mathfrak{X}$ induced on $\nu_{0}(\mathfrak{X}) u$ is a direct sum of irreducible representations each of which belongs to $\mathfrak{D}_{\Lambda_{0}}$. Since $u$ belongs to the highest weight $\Lambda_{0}$, it follows that $\nu\left(\mathfrak{Y}_{\Lambda_{0}}\right) u=0$ and therefore $\nu\left(\mathfrak{H}_{\mathrm{A}_{0}}\right) u=0$. Given any $v \in A$ we can, by Lemma 27 , find a $b \in \mathfrak{B}$ such that $\nu(b)\left(1 \times 1^{*}\right)=v$. If $b^{\prime}$ is another such element then by the same lemma, $b-b^{\prime} \in \mathfrak{R Y}_{\Lambda_{0}}$ and therefore $\nu\left(b-b^{\prime}\right) u=0$. Hence $\nu(b) u$ is uniquely determined by $v$ alone. Now we define multiplication by setting $v u=\nu(b) u$. If $b^{\prime}$ is any element in $\mathfrak{B}$ such that $\nu\left(b^{\prime}\right)\left(1 \times 1^{*}\right)=u$, then $v u=\nu(b) \nu\left(b^{\prime}\right)\left(1 \times 1^{*}\right)$. This immediately shows that the multiplication is associative. For any $z \in \mathfrak{C}, \nu(z)\left(1 \times 1^{*}\right)=z \times 1^{*} \in A$. Also if $z \neq 0$ then $z \times 1^{*} \neq 0$. Therefore the mapping $z \rightarrow z \times 1^{*}(z \in \mathbb{C})$ is clearly an isomorphism of $\mathbb{E}$ into $A$. We may therefore identify $\mathbb{E}$ with its image under this mapping. Then it is easily seen that $\mathbb{C}$ lies in the center of $A$ and $A$ is a finite $\mathbb{E}$-module (under the

$\left.{ }^{17}\right)$ We denote the natural mapping of $\mathfrak{X}$ on $\mathfrak{X}^{*}$ by $x \rightarrow x^{*}(x \in \mathfrak{X})$ throughout. 
multiplication defined in $A$ ). Let $及$ be the center of $\mathfrak{B}$. We note for later use that if $z \in \Re, \nu(z)\left(1 \times 1^{*}\right)$ lies in the center of $A$.

Now let $\chi$ be any homomorphism of $\Subset$ into $C$ such that $\chi(1)=1$. Let $\mathfrak{N}_{\chi}$ be the left ideal in $\mathfrak{A}$ generated by all elements of the form $z-\chi(z)(z \in \mathbb{S})$. Then it is obvious that $\mathfrak{N}_{\chi}$ is actually an ideal and therefore $\left[X, \mathfrak{N}_{\chi}\right]$ $=\left[\gamma(X), \mathfrak{N}_{\chi}\right] \subset \mathfrak{N}_{\chi}\left(X \in \mathfrak{R}_{K}\right)$. Therefore the space $\mathfrak{N}_{\chi} \mathbf{X} \mathfrak{X}^{*}$ is invariant under $\nu(\mathfrak{B})$. Let $z \rightarrow \bar{z}$ denote the natural mapping of $\mathfrak{A}$ on $\overline{\mathfrak{A}}=\mathfrak{A} / \mathfrak{N}_{\chi}$ and $\theta$ the natural mapping of $\mathfrak{A} \times \mathfrak{X}^{*}$ on the factor space $\left(\mathfrak{A} \times \mathfrak{X}^{*}\right) /\left(\mathfrak{N}_{\chi} \times \mathfrak{X}^{*}\right)$. Then it is easily seen that $\overline{\mathfrak{X}} \mathbf{X} \mathfrak{X}^{*}$ and $\left(\mathfrak{Y} X \mathfrak{X}^{*}\right) /\left(\mathfrak{N}_{\chi} \mathfrak{X}^{*}\right)$ are isomorphic under the mapping $\bar{z} \times x^{*} \leftrightarrow \theta\left(z \times x^{*}\right) \quad(z \in \mathfrak{A}, x \in \mathfrak{X})$. We therefore identify the two spaces under this mapping. Let $\bar{\nu}$ be the representation of $\mathfrak{B}$ induced on $\left(\overline{\mathfrak{Y}} \times \mathfrak{X}^{*}\right)$ $=\theta\left(\mathfrak{A} \times \mathfrak{X}^{*}\right)$. Then it is easy to verify that

$$
\begin{aligned}
& \bar{\nu}(X)\left(\bar{z} \times \omega^{*}\right)=[\overline{X, z}] \times \omega^{*}+z \times(X \omega)^{*}, \\
& \bar{\nu}(w)\left(\bar{z} \times \omega^{*}\right)=\bar{w} \bar{z} \times \omega^{*} \quad\left(X \in \mathfrak{\Omega}_{K}, z, w \in \mathfrak{U}, \omega \in \mathfrak{X}\right) .
\end{aligned}
$$

Also it is clear that

$$
\left(\overline{\mathfrak{A}} \times \mathfrak{X}^{*}\right)_{\Lambda} \supset \theta\left(\left(\mathfrak{U} \times \mathfrak{X}^{*}\right)_{\Lambda}\right) \quad\left(\Lambda \in \mathrm{P}_{K}\right) .
$$

Since $\mathfrak{A} \times \mathfrak{X}^{*}=\sum_{\Lambda}\left(\mathfrak{A} \times \mathfrak{X}^{*}\right)_{\Lambda}$, it follows that

$$
\overline{\mathfrak{Y}} \times \mathfrak{X}=\sum_{\Lambda}(\overline{\mathfrak{Y}} \times \mathfrak{X})_{\Lambda}
$$

and $\left(\overline{\mathfrak{Y}} \times \mathfrak{X}^{*}\right)_{\Lambda}=\theta\left(\left(\mathfrak{Y} \times \mathfrak{X}^{*}\right)_{\Lambda}\right)$. We have seen that $\left(\mathfrak{H} \times \mathfrak{X}^{*}\right)_{\Lambda}$ is a finite module over $\mathfrak{S}$. Since $\bar{\nu}(z-\chi(z))=0(z \in \mathfrak{S})$ it follows that $\operatorname{dim}\left(\overline{\mathfrak{Y}} \times \mathfrak{X}^{*}\right)_{\Lambda}<\infty$. Now consider $A \cap\left(\mathfrak{N}_{\chi} \times \mathfrak{X}^{*}\right)$. Since $\mathfrak{N}_{\chi} \times \mathfrak{X}^{*}$ is invariant under $\nu(\mathfrak{B}), A \cap\left(\mathfrak{N}_{\chi} \times \mathfrak{X}^{*}\right)$ is a left ideal in $A$. We shall now prove that it is actually an ideal in $A$. First notice that $\mathfrak{N X _ { \chi }}$ is an ideal in $\mathfrak{B}$ since $\left[X, \mathfrak{N}_{\chi}\right] \subset \mathfrak{N}_{\chi}\left(X \in \mathfrak{R}_{K}\right)$ and $\mathfrak{A} \mathfrak{X}=\mathfrak{X} \mathfrak{A}=\mathfrak{B}$. Let $u \in A \cap\left(\mathfrak{N}_{\chi} \times \mathfrak{X}^{*}\right), v \in A$. Then we can find $b \in \mathfrak{N}_{\chi} \mathfrak{X}, b^{\prime} \in \mathfrak{B}$ such that $u$ $=\nu(b)\left(1 \times 1^{*}\right), v=\nu\left(b^{\prime}\right)\left(1 \times 1^{*}\right)$. Hence $u v=\nu\left(b b^{\prime}\right)\left(1 \times 1^{*}\right)$. Since $\mathfrak{N}_{\chi} \mathfrak{X}$ is an ideal, $b b^{\prime} \in \mathfrak{N}_{\chi} \mathfrak{X}$ and therefore $u v \in\left(\mathfrak{N}_{\chi} \times \mathfrak{X}^{*}\right) \cap A$. Put $\bar{A}=\theta(A)$. Then we can regard $\bar{A} \cong A / A \cap\left(\mathfrak{N}_{\chi} \times \mathfrak{X}^{*}\right)$ as a factor algebra of $A$. Again we verify that if $\bar{\nu}\left(b_{1}\right)\left(\overline{1} \times 1^{*}\right)=\bar{u}_{1}, \quad \bar{\nu}\left(b_{2}\right)\left(\overline{1} \times 1^{*}\right)=\bar{u}_{2} \quad$ then $\quad \bar{u}_{1} \bar{u}_{2}=\bar{\nu}\left(b_{1} b_{2}\right)\left(\overline{1} \times 1^{*}\right) \quad\left(b_{1}, b_{2} \in \mathfrak{B}\right.$, $\left.\bar{u}_{1}, \bar{u}_{2} \in A\right)$. Since $\operatorname{dim} \bar{A} \leqq \operatorname{dim}\left(\overline{\mathfrak{Y}} \times \mathfrak{X}^{*}\right)_{\Lambda_{0}}<\infty, \bar{A}$ is a finite-dimensional associative algebra. We note that $\overline{1} \times 1^{*}$ is the unit element of $\bar{A}$.

Put $\mathfrak{M}_{\Lambda_{0}}=\mathfrak{Y} \mathfrak{Y}_{\Lambda_{0}}+\mathfrak{N}_{\chi} \mathfrak{X}$. It follows easily from Lemma 27 that $\bar{\nu}(\mathfrak{B})\left(\overline{1} \times 1^{*}\right)$ $=\overline{\mathfrak{Y}} \times \mathfrak{X}^{*}$ and $\nu(b)\left(\overline{1} \times 1^{*}\right)=0(b \in \mathfrak{B})$ if and only if $b \in \mathfrak{M}_{\Lambda_{0}}$.

Lemma 28. Let $\mathfrak{M}$ be a maximai left ideal in $\mathfrak{B}$ such that $\mathfrak{M} \supset \mathfrak{M}_{\Lambda_{0}}$. Put $\mathfrak{M}_{*}=\bar{\nu}(\mathfrak{M})\left(\overline{1} \times 1^{*}\right)$. Then $\mathfrak{M}_{*} \cap \bar{A}$ is a maximal left ideal in $\bar{A}$.

Let $z \rightarrow z_{*}$ denote the natural mapping of $\mathfrak{B}$ on $\mathfrak{B}_{*}=\mathfrak{B} / \mathfrak{M}_{\Lambda_{0}}$ and let $\pi_{*}$ be the natural representation of $\mathfrak{B}$ on $\mathfrak{B}_{*}$. It is obvious that $\bar{\nu}$ is equivalent to $\pi_{*}$ under the isomorphism $\bar{\nu}(b)\left(\overline{1} \times 1^{*}\right) \leftrightarrow b_{*}(b \in \mathfrak{B})$ of $\overline{\mathfrak{A}} \times \mathfrak{X}^{*}$ with $\mathfrak{B}_{*}$. We may 
therefore identify these two spaces under this isomorphism. Since $\mathfrak{M}$ is a left ideal it is clear that $\mathfrak{M}_{*}$ is invariant under $\bar{\nu}(\mathfrak{B})$ and therefore $\mathfrak{M}_{*} \cap \bar{A}$ is a left ideal in $\bar{A}$. Also $\overline{1} \times 1^{*} \in \mathfrak{M}_{*} \cap \bar{A}$ for otherwise we would have $\mathfrak{B}_{*}=\bar{\nu}(\mathfrak{B})$ $\left(\overline{1} \times 1^{*}\right) \subset \mathfrak{M}_{*}$. Since $\mathfrak{M} \supset \mathfrak{M}_{\Lambda_{0}}$ this would imply that $\mathfrak{B} / \mathfrak{M} \cong \mathfrak{B}_{*} / \mathfrak{M}_{*}=\{0\}$ thus contradicting the fact that $\mathfrak{M}$ is maximal.

Let $\rho$ be the natural representation of $\mathfrak{B}$ on $\mathfrak{B} / \mathfrak{M}$. Since $\mathfrak{M}$ is maximal $\rho$ is irreducible. Also since $\mathfrak{M} \supset \mathfrak{M}_{\Lambda_{0}}, \rho$ is equivalent to the representation induced by $\bar{\nu}$ on $\mathfrak{B}_{*} / \mathfrak{M}_{*}$. Let $u, v \in \bar{A}, u \notin \mathfrak{M}_{*}$. Since $\rho$ is irreducible we can find a $b \in \mathfrak{B}$ such that $\bar{\nu}(b) u \equiv v \bmod \mathfrak{M}_{*}$. We have seen above (equation (9), p. 63) that $\overline{\mathfrak{Y}} \times \mathfrak{X}^{*}=\sum_{\Lambda}\left(\overline{\mathfrak{Y}} \times \mathfrak{X}^{*}\right)_{\Lambda}$. Hence $\bar{\nu}(b) u=u_{0}+u_{1}+\cdots+u_{r}$ where $u_{i} \in\left(\overline{\mathfrak{Y}} \times \mathfrak{X}^{*}\right)_{\Lambda_{i}}, \Lambda_{0}, \cdots, \Lambda_{r}$ being distinct. Then

$$
\bar{\nu}(b) u-v=\left(u_{0}-v\right)+u_{1}+\cdots+u_{r} \in \mathfrak{M}_{*} .
$$

From Lemma 4 of $[9$, p. 912], we can find an $x \in \mathfrak{X}$ such that $\bar{\nu}(x) z=0$ for all $z \in\left(\overline{\mathfrak{A}} \times \mathfrak{X}^{*}\right)_{\Lambda_{i}}, 1 \leqq i \leqq r$, and $\bar{\nu}(x) z=z$ for all $z \in\left(\overline{\mathfrak{A}} \times \mathfrak{X}^{*}\right)_{\Lambda_{0}}$. Hence

$$
\bar{\nu}(x)\{\bar{\nu}(b) u-v\}=\bar{\nu}(x b) u-v=u_{0}-v \in \mathfrak{M}_{*} \text {. }
$$

Further if $\lambda_{0}, \lambda_{1}, \cdots, \lambda_{s}\left(\lambda_{0}=\Lambda_{0}\right)$ are all the distinct weights of $\mathfrak{D}_{\Lambda_{0}}$, put

$$
x^{\prime}=\frac{\prod_{1 \leqq i \leqq s}\left(H-\lambda_{i}(H)\right)}{\prod_{1 \leqq i \leqq s}\left(\lambda_{0}(H)-\lambda_{i}(H)\right)}
$$

where $H$ is any element in $\mathfrak{h}_{K}$ such that $\lambda_{0}(H) \neq \lambda_{i}(H), 1 \leqq i \leqq s$. Then clearly $\nu\left(x^{\prime}\right) z \in \bar{A}$ for any $z \in\left(\overline{\mathfrak{A}} \times \mathfrak{X}^{*}\right)_{\Lambda_{0}}$ and $\bar{\nu}\left(x^{\prime}\right) z=z$ if $z \in \bar{A}$. Hence

$$
\bar{\nu}\left(x^{\prime} x b\right) u-v=\nu\left(x^{\prime}\right)\left(u_{0}-v\right) \in \mathfrak{M}_{*} \cap \bar{A} .
$$

Put $b^{\prime}=x^{\prime} x b$. Then $\bar{\nu}\left(b^{\prime}\right) u-v \in \mathfrak{M}_{*} \cap \bar{A}$ and since $v \in \bar{A}, \bar{\nu}\left(b^{\prime}\right) u \in \bar{A}$. Let $\mathfrak{M}_{u}$ be the left ideal in $\mathfrak{B}$ consisting of all $z \in \mathfrak{B}$ such that $\bar{\nu}(z) u=0$. It is clear that $\mathfrak{M}_{u} \supset \mathfrak{M}_{\Lambda_{0}}$. Let $\mathfrak{M}_{u *}=\bar{\nu}\left(\mathfrak{M}_{u}\right)\left(\overline{1} \times 1^{*}\right)$ and let $\theta$ be the natural mapping of $\mathfrak{B}_{*}$ on $\mathfrak{B}_{*} / \mathfrak{M}_{u *}$. Also let $\bar{\nu}_{0}$ be the representation of $\mathfrak{B}$ on $\mathfrak{B}_{*} / \mathfrak{M}_{u *}$ induced by $\bar{\nu}$. Then it is obvious that

$$
\bar{\nu}(\mathfrak{B}) u \cong \mathfrak{B} / \mathfrak{M}_{u} \cong \mathfrak{B}_{*} / \mathfrak{M}_{u *}=\left(\overline{\mathfrak{A}} \times \mathfrak{X}^{*}\right) / \mathfrak{M}_{u *}
$$

and the representation of $\mathfrak{B}$ on $\bar{\nu}(\mathfrak{B}) u$ is equivalent to $\bar{\nu}_{0}$ under the isomorphism $\bar{\nu}(z) u \leftrightarrow \theta\left(\bar{\nu}(z)\left(\overline{1} \times 1^{*}\right)\right)(z \in \mathfrak{B})$ of $\bar{\nu}(\mathfrak{B}) u$ with $\mathfrak{B}_{*} / \mathfrak{M}_{u *}$. Since $\bar{\nu}\left(b^{\prime}\right) u \in \bar{A}$ it follows that

$$
\theta\left(\bar{\nu}\left(b^{\prime}\right)\left(\overline{1} \times 1^{*}\right)\right) \in\left(\mathfrak{B}_{*} / \mathfrak{M}_{u *}\right)_{\Lambda_{0}, \Lambda_{0}}
$$

in the notation of Lemma 23. But it is obvious that

$$
\left(\mathfrak{B}_{*} / \mathfrak{M}_{u *}\right)_{\Lambda_{0}, \Lambda_{0}}=\theta(\bar{A}) .
$$

Hence $\bar{\nu}\left(b^{\prime}\right)\left(\overline{1} \times 1^{*}\right) \in \bar{A}+\mathfrak{M}_{u *}$. Therefore we can find a $z \in \mathfrak{M}_{u}$ such that 
$\bar{\nu}\left(b^{\prime}-z\right)\left(\overline{1} \times 1^{*}\right)=u^{\prime} \in \bar{A}$. But then

$$
u^{\prime} u-v=\bar{\nu}\left(b^{\prime}-z\right) u-v=\bar{\nu}\left(b^{\prime}\right) u-v \in \mathfrak{M}_{*} \cap \bar{A}
$$

since $z \in \mathfrak{M}_{u}$. Since $u, v$ were any two elements in $\bar{A}$ such that $u \in \mathfrak{M}_{*} \cap \bar{A}$, this shows that the natural representation of $\bar{A}$ on $\bar{A} / \mathfrak{M}_{*} \cap \bar{A}$ is irreducible. Since $\overline{1} \times 1^{*} \notin \mathfrak{M}_{*} \cap A$ it follows that $\mathfrak{M}_{*} \cap \bar{A}$ is a maximal left ideal in $\bar{A}$.

We have seen above that every maximal left ideal $\mathfrak{M}$ in $\mathscr{B}$ such that $\mathfrak{M} \supset \mathfrak{M}_{\Lambda_{0}}$ defines an irreducible representation of $\bar{A}$ namely the natural representation of $\bar{A}$ on $\bar{A} / \mathfrak{M}_{*} \cap \bar{A}$. Since $\bar{A}$ is a finite-dimensional associative algebra it has only a finite number of inequivalent irreducible representations. Hence there exist a finite number of maximal left ideals $\mathfrak{M}_{i}, 1 \leqq i \leqq r$, in $\mathfrak{B}$ each containing $\mathfrak{M}_{\Lambda_{0}}$ such that if $\mathfrak{M}$ is any maximal left ideal containing $\mathfrak{M}_{\Lambda_{0}}$ the representation of $\bar{A}$ defined by $\mathfrak{M}$ is equivalent to the one defined by $\mathfrak{M}_{i}$ for some $i, 1 \leqq i \leqq r$. Let $\rho_{i}$ denote the natural representation of $\mathfrak{B}$ on $\mathfrak{B} / \mathfrak{M}_{i}$.

Lemma 29. Let $\mathfrak{M}$ be a maximal left ideal in $\mathfrak{B}$ such that $\mathfrak{M} \supset \mathfrak{M}_{\Lambda_{0}}$ and let $\rho$ be the natural representation of $\mathfrak{B}$ on $\mathfrak{B} / \mathfrak{M}$. Then $\rho$ is equivalent to $\rho_{i}$ for some $i, 1 \leqq i \leqq r$. Moreover if 3 is the center of $B$ there exists a homomorphism $\xi$ of $\mathbb{Z}$ into $C$ such that $z-\xi(z) \in \mathfrak{M}$ for all $z \in \mathfrak{Z}$.

We keep to the notation of the proof of the preceding lemma. Then $\mathfrak{M}_{*}=\bar{\nu}(\mathfrak{M})\left(\overline{1} \times 1^{*}\right), \mathfrak{M}_{i *}=\bar{\nu}\left(\mathfrak{M}_{i}\right)\left(\overline{1} \times 1^{*}\right), 1 \leqq i \leqq r$, and we can choose an $i$ such that the natural representations of $\bar{A}$ on $\bar{A} / \mathfrak{M}_{*} \cap \bar{A}$ and $\bar{A} / \mathfrak{M}_{i *} \cap \bar{A}$ are equivalent. Hence we can find an element $v \in \bar{A}\left(v \notin \mathfrak{M}_{*} \cap \bar{A}\right)$ such that for any $u \in \bar{A}, u v \in \mathfrak{M}_{*}$ if and only if $u \in \mathfrak{M}_{i *} \cap \bar{A}$. Let $\mathfrak{M}_{v}$ be the left ideal in $\mathfrak{B}$ consisting of all $b \in \mathfrak{B}$ such that $\bar{\nu}(b) v \in \mathfrak{M}_{*}$. Let $\beta$ be the natural mapping of $\mathfrak{B}$ on $\mathfrak{B} / \mathfrak{M}$ and $\theta$ the natural mapping of $\mathfrak{B}_{*}$ on $\mathfrak{B}_{*} / \mathfrak{M}_{*}$. Since $\mathfrak{M} \supset \mathfrak{M}_{\Lambda_{0}}, \rho$ is equivalent to the representation of $\mathfrak{B}$ on $\mathfrak{B}_{*} / \mathfrak{M}_{*}$ induced by $\bar{\nu}$, under the natural isomorphism

$$
\beta(b) \leftrightarrow \theta\left(b_{*}\right)=\theta\left(\bar{\nu}(b)\left(\overline{1} \times 1^{*}\right)\right)
$$

of $\mathfrak{B} / \mathfrak{M}$ with $\mathfrak{B}_{*} / \mathfrak{M}_{*}$. Since $v \notin \mathfrak{M}, \theta(v) \neq 0$. Hence $\rho(\mathfrak{B}) \theta(v)=\mathfrak{B} / \mathfrak{M}$ since $\rho$ is irreducible. Let $\mathfrak{M}^{\prime}$ be the set of all elements $b \in \mathfrak{B}$ such that $\rho(b) \theta(v)=0$. Clearly $\mathfrak{M}^{\prime}$ is a maximal left ideal in $\mathfrak{B}$ and if $\rho^{\prime}$ is the natural representation of $\mathscr{B}$ on $\mathfrak{B} / \mathfrak{M}^{\prime}, \rho$ is equivalent to $\rho^{\prime}$ under the isomorphism $\beta(b a) \leftrightarrow \beta^{\prime}(b) \quad(b \in \mathfrak{B})$ where $\beta^{\prime}$ is the natural mapping of $\mathfrak{B}$ on $\mathfrak{B} / \mathfrak{M}^{\prime}$ and $a$ is any element in $\mathfrak{B}$ such that $\beta(a)=\theta(v)$. Hence it is sufficient to prove that $\rho^{\prime}$ is equivalent to $\rho_{i}$.

Let $\mathfrak{M}_{*}^{\prime}=\bar{\nu}\left(\mathfrak{M}^{\prime}\right)\left(\overline{1} \times 1^{*}\right)$. Then $u \in \mathfrak{M}_{*}^{\prime} \cap \bar{A}$ if and only if $u \in \bar{A}$ and $u v \in \mathfrak{M}_{*}$, that is, if and only if $u \in \mathfrak{M}_{i *} \cap \bar{A}$. Hence $\mathfrak{M}_{*}^{\prime} \cap \bar{A}=\mathfrak{M}_{i *} \cap \bar{A}$. We now claim that $\mathfrak{M}^{\prime}=\mathfrak{M}_{i}$. For otherwise suppose $\mathfrak{M}^{\prime} \neq \mathfrak{M}_{i}$. Since they are both maximal left ideals, $1 \in \mathfrak{M}^{\prime}+\mathfrak{M}_{i}$. Hence $\overline{1} \times 1^{*}=z_{*}+w_{*}$ where $z_{*} \in \mathfrak{M}_{*}^{\prime}, w_{*} \in \mathfrak{M}_{i *}$. Since $\mathfrak{M}_{*}^{\prime}$ and $\mathfrak{M}_{i *}$ are invariant under $\bar{\nu}(\mathfrak{X})$, it follows from Lemmas 1,22 , and 23 and the relation $\overline{\mathfrak{Y}} \times \mathfrak{X}^{*}=\sum_{\Lambda}\left(\overline{\mathfrak{A}} \times \mathfrak{X}^{*}\right)_{\Lambda}$, that 


$$
\left.\mathfrak{M}_{*}^{\prime}=\sum_{\Lambda, \mu} \mathfrak{M}_{*}^{\prime} \cap \overline{(\mathfrak{R}} \times \mathfrak{X}^{*}\right)_{\Lambda, \mu}
$$

in the notation of Lemma 23. A similar equation holds for $\mathfrak{M}_{i *}$. Since the sum $\sum_{\Lambda, \mu}\left(\overline{\mathfrak{A}} \times \mathfrak{X}^{*}\right)_{\Lambda, \mu}$ is direct and $\overline{1} \times 1^{*} \in \bar{A}=\left(\overline{\mathfrak{I}} \times \mathfrak{X}^{*}\right)_{\Lambda_{0}, \Lambda_{0}}$ it follows that $\overline{1} \times 1^{*}$ $=z_{*}^{\prime}+w_{*}^{\prime}$ where $z_{*}^{\prime}$ and $w_{*}^{\prime}$ respectively are the components of $z_{*}$ and $w_{*}$ in $\bar{A}$. From the above remarks it is clear that $z_{*}^{\prime} \in \mathfrak{M}_{*}^{\prime} \cap \bar{A}, w_{*}^{\prime} \in \mathfrak{M}_{i *} \cap \bar{A}$. But since $\mathfrak{M}_{*}^{\prime} \cap \bar{A}=\mathfrak{M}_{i *} \cap \bar{A}$, we have $\overline{1} \times 1^{*} \in \mathfrak{M}_{i *} \cap \bar{A}$ which of course is false since $\mathfrak{M}_{i *} \cap \bar{A} \neq \bar{A}$ from Lemma 28 . Therefore $\mathfrak{M}_{i}=\mathfrak{M}^{\prime}$ and $\rho_{i}=\rho^{\prime}$.

Furthermore we know that $\bar{\nu}(z)\left(\overline{1} \times 1^{*}\right)$ lies in the center of $\bar{A}$ for any $z \in Z$. Since the natural representation of $\bar{A}$ on $\bar{A} / \bar{A} \cap \mathfrak{M}_{*}$ is irreducible it follows from Schur's lemma that we can find $\xi(z) \in C$ such that $\bar{\nu}(z)\left(\overline{1} \times 1^{*}\right)$ $-\xi(z)\left(\overline{1} \times 1^{*}\right) \in \bar{A} \cap \mathfrak{M}_{*}$. Hence $z-\xi(z) \in \mathfrak{M}$. Since the mapping $z \rightarrow \xi(z)(z \in \mathfrak{Z})$ is clearly a homomorphism the lemma is proved completely.

It is now easy to deduce Theorem 4 . Let $V$ be the representation space of $\pi$. Since by hypothesis $V_{\Lambda_{0}} \neq\{0\}$ we can find an element $\psi \in V_{\Lambda_{0}}, \psi \neq 0$. We may clearly suppose that the space $\pi(\mathfrak{X}) \psi$ is irreducible under $\pi(\mathfrak{X})$ and $\psi$ belongs to the highest weight $\Lambda_{0}$. Then it follows that $\pi\left(\mathfrak{V}_{\Lambda_{0}}\right) \psi=0$. Let $\mathfrak{M}$ be the set of all elements $b \in \mathfrak{B}$ such that $\pi(b) \psi=0$. Since $\pi$ is irreducible, $\mathfrak{M}$ is a maximal left ideal and $\mathfrak{M} \supset \mathfrak{H} \mathfrak{Y}_{\Lambda_{0}}$. Also it is clear that $\mathfrak{M} \supset \mathfrak{N}_{x}$ and therefore $\mathfrak{M} \supset \mathfrak{X} \mathfrak{P}_{\mathfrak{x}_{x}}=\mathfrak{M}_{x} \mathfrak{X}$. Hence $\mathfrak{M} \supset \mathfrak{M}_{\Lambda_{0}}=\mathfrak{Y} \mathfrak{Y}_{\Lambda_{0}}+\mathfrak{R}_{x} \mathfrak{X}$. Let $\rho$ be the natural representation of $\mathfrak{B}$ on $\mathfrak{B} / \mathfrak{M}$. Then from Lemma $29, \rho$ is equivalent to $\rho_{i}$ for some $1 \leqq i \leqq r$. Since $\pi$ is clearly equivalent to $\rho$ the first assertion of the theorem is established.

Now we come to the second part. We have already seen (cf. p. 63) that $\operatorname{dim}\left(\overline{\mathfrak{V}} \times \mathfrak{X}^{*}\right)_{\Lambda}<\infty$ and $\mathfrak{B} / \mathfrak{M}=\theta\left(\overline{\mathfrak{A}} \times \mathfrak{X} \mathfrak{X}^{*}\right)=\sum_{\Lambda} \theta\left(\left(\mathfrak{Y} \times \mathfrak{X} \mathfrak{X}^{*}\right)_{\Lambda}\right)$. Let $(\mathfrak{B} / \mathfrak{M})_{\Lambda}$ denote the set of all elements of $\mathfrak{B} / \mathfrak{M}$ which transform under $\rho$ according to $\mathfrak{D}_{\Lambda}$. Then it is easily seen that $(\mathfrak{B} / \mathfrak{M})_{\Lambda}=\theta\left(\left(\mathfrak{I} X \mathfrak{X}^{*}\right)_{\Lambda}\right)$. Hence

$$
\operatorname{dim}(\mathfrak{B} / \mathfrak{M})_{\Lambda} \leqq \operatorname{dim}\left(\overline{\mathfrak{A}} \times \mathfrak{X}^{*}\right)_{\Lambda}<\infty .
$$

Since the existence of the homomorphism $\xi$ has already been established in Lemma 29, the proof of Theorem 4 is now complete.

Part III. Characters. Let $\&$ be a semisimple Lie algebra over $C$ and $\mathfrak{B}$ the universal enveloping algebra of $\mathfrak{R}$. Choose a fixed Cartan subalgebra $\mathfrak{h}$ of $\mathfrak{R}$ and a fundamental system of roots $\left\{\alpha_{1}, \cdots, \alpha_{l}\right\}$ of $\mathfrak{R}$ with respect to $\mathfrak{h}$. Let $\mathbb{B}$ denote the center of $\mathfrak{B}$.

Definition. A complex-valued linear function $\chi$ on $\mathfrak{B}$ will be called a character if the following conditions are fulfilled:

(1) $\chi\left(b_{1} b_{2}\right)=\chi\left(b_{2} b_{1}\right)$ for all $b_{1}, b_{2} \in \mathfrak{B}$.

(2) $\chi(1)=1$ and $\chi\left(z_{1} z_{2}\right)=\chi\left(z_{1}\right) \chi\left(z_{2}\right)$ for all $z_{1}, z_{2} \in 3$.

Let $X_{i}, 1 \leqq i \leqq n$, be a base for $\mathbb{R}$. Put $g_{i j}=\operatorname{sp}\left(\operatorname{ad} X_{i}\right.$ ad $\left.X_{j}\right)$. Since $\&$ is semisimple the matrix $\left(g_{i j}\right)_{1 \leqq i, j \leqq n}$ is nonsingular. Let $\left(g^{i j}\right)_{1 \leqq i, j \leqq n}$ denote its inverse. Put $X^{i}=\sum_{1 \leqq j \leqq n} g^{i j} X_{j}$. 
Lemma 30. If $\chi$ is a character and $\left(j_{1}, \cdots, j_{r}\right)$ is any permutation of the set $(1,2, \cdots, r)$, then

$$
\sum_{1 \leqq i_{1}, \cdots, i_{r} \leqq n} \chi\left(X_{i_{1}} X_{i_{2}} \cdots X_{i_{r}}\right) X^{i_{j_{1}}} X^{i i_{2}} \cdots X^{i_{i_{r}}} \in \Re .
$$

The proof of this lemma is exactly parallel to a similar assertion proved in [9, p. 912].

LEMma 31. If $\chi_{1}$ and $\chi_{2}$ are two distinct characters of $\mathfrak{B}$ we can find $a z \in \mathbb{Z}$ such that $\chi_{1}(z) \neq \chi_{2}(z)$.

We know that the basic canonical elements $\left({ }^{18}\right) S_{\left(i_{1}, \cdots, i_{r}\right)} X_{i_{1}} X_{i_{2}} \cdots X_{i_{r}}$, $1 \leqq i_{1}, \cdots, i_{r} \leqq n, r \geqq 0$, form a base for $\mathfrak{B}$. Since $\chi_{1} \neq \chi_{2}$ we can find an $r \geqq 0$ and $1 \leqq i_{1}, \cdots, i_{r} \leqq n$ such that

$$
\chi_{1}\left(\underset{\left(i_{1}, \cdots, i_{r}\right)}{S} X_{i_{1}} X_{i_{2}} \cdots X_{i_{r}}\right) \neq \chi_{2}\left(\underset{\left(i_{1}, \cdots, i_{r}\right)}{S} X_{i_{1}} X_{i_{2}} \cdots X_{i_{r}}\right) .
$$

Put

$$
\begin{array}{r}
a_{j_{1} j_{2} \cdots j_{r}}=\chi_{1}\left(\underset{\left(j_{1}, \cdots, i_{r}\right)}{S} X_{j_{1}} \cdots X_{i_{r}}\right)-\chi_{2}\left(\underset{\left(j_{1}, \cdots, j_{r}\right)}{S} X_{j_{1}} \cdots X_{j_{r}}\right), \\
1 \leqq j_{1}, \cdots, j_{r} \leqq n .
\end{array}
$$

Then $a_{j_{1} j_{2} \ldots j_{r}}$ are symmetric and not all of them are zero. Hence

$$
w=\sum_{1 \leqq j_{1}, \cdots, j_{r} \leqq n} a_{j_{1} j_{2} \cdots j_{r}} X^{j_{1}} X^{j_{2}} \cdots X^{j_{r}} \neq 0
$$

(cf. [9, p. 913]). Also from Lemma 30, weß. Hence from Theorem 1 of [9, p. 905] we can find a finite-dimensional representation $\pi^{\prime}$ of $\mathfrak{B}$ such that $\pi^{\prime}(w) \neq 0$. Since $\mathfrak{R}$ is semisimple $\pi^{\prime}$ is fully reducible. Hence $\pi(w) \neq 0$ for some irreducible component $\pi$ of $\pi^{\prime}$. Since $\pi$ is irreducible and $w \in \Re$, it follows from Schur's lemma that $\pi(w)=c \pi(1)(c \in C)$. Then $c \neq 0$ and therefore sp $\pi(w) \neq 0$. Now put

$$
z=\sum_{1 \leqq j_{1}, \cdots, j_{r} \leqq n}\left\{\operatorname{sp} \pi\left(\underset{\left(j_{1}, \cdots, j_{r}\right)}{S} X_{j_{1}} X_{j_{2}} \cdots X_{j_{r}}\right)\right\} X^{i_{1}} X^{j_{2}} \cdots X^{i_{r}}
$$

If $d$ is the degree of $\pi$ the function sp $\pi(b) / d(b \in \mathfrak{B})$ is clearly a character. Hence from Lemma 30, $z \in \Re$. Also

$$
\chi_{1}(z)-\chi_{2}(z)=\operatorname{sp} \pi(w) \neq 0 .
$$

Hence the lemma is proved.

The above lemma shows that a character is uniquely determined by its value on $\mathbb{3}$. Our object is to obtain all the characters of $\mathfrak{B}$.

Let $P$ be the set of all dominant integral functions on $\mathfrak{h}$. For any $\Lambda \in P$ let $\pi_{\Lambda}$ denote a finite-dimensional irreducible representation of $\mathfrak{B}$ with the

$\left.{ }^{18}\right)$ The symbol $\left.S_{\left(i_{1}\right.}, \ldots, i_{r}\right)$ has the same meaning as on p. 51. 
highest weight $\Lambda$ and let $d_{\Lambda}$ be the degree of $\pi_{\Delta}$. For any root $\alpha$ let $X_{\alpha} \neq 0$ be the root element corresponding to $\alpha$. Put $\left[X_{\alpha}, X_{-\alpha}\right]=H_{\alpha}, H_{i}=H_{\alpha_{i}}, 1 \leqq i \leqq l$. As before we may assume that $\alpha\left(H_{\alpha}\right)=2$. Let $\mathfrak{S}$ be the subalgebra of $\mathfrak{B}$ generated by $H_{i}, 1 \leqq i \leqq l$, and 1 , and let $C[x]$ be the (commutative) ring of all polynomials in $l$ independent variables $x_{1}, \cdots, x_{l}$ with coefficients in $C$. We know that $H_{1}^{m_{1}} H_{2}^{m_{2}} \cdots H_{l}^{m_{l}}, m_{1}, \cdots, m_{l} \geqq 0$, are linearly independent. Let $\beta$ denote the isomorphism of $\mathfrak{S}$ onto $C[x]$ defined by $\beta\left(H_{1}^{m_{1}} \cdots H_{l}^{m_{l}}\right)$ $=x_{1}^{m_{1}} \cdots x_{l}^{m_{l}}, m_{1}, \cdots, m_{l} \geqq 0$. Moreover if $\lambda$ is any linear function on $\mathfrak{h}$ and $f(x) \in C[x]$ we denote by $f(\lambda)$ the value of $f(x)$ at the point $x_{i}=\lambda\left(H_{i}\right)$, $1 \leqq i \leqq l$. We shall constantly make use of the following simple lemma which is easily proved by induction on $l$.

Lemma 32. If $f \in C[x](f \neq 0)$ we can find $l$ integers $\lambda_{1}, \cdots, \lambda_{l}$ all greater than or equal to 0 such that $f\left(\lambda_{1}, \cdots, \lambda_{l}\right) \neq 0$.

Put $\mathfrak{B}=\sum_{\alpha>0} \mathfrak{B} X_{\alpha}$. First we prove a few preliminary lemmas.

LEMMA 33. $\mathfrak{B} \cap \mathfrak{S}=\{0\}$.

Let $h \in \mathfrak{B} \cap \mathfrak{S}$. Suppose $h \neq 0$. Then $\beta(h)=f(x) \neq 0$. Hence from Lemma 32 , we can find a $\Lambda \in \mathrm{P}$ such that $f(\Lambda) \neq 0$. Let $\psi \neq 0$ be a vector belonging to the highest weight $\Lambda$ in the representation space of $\pi_{\Lambda}$. Then clearly $\pi_{\Lambda}(h) \psi$ $=f(\Lambda) \psi \neq 0$. On the other hand since $h \in \mathfrak{B}, \pi_{\Delta}(h) \psi=0$. Thus we get a contradiction and the lemma is proved.

We recall that the adjoint representation $\rho$ of $\mathfrak{B}$ is defined by the relation $\rho(X) b=[X, b](X \in \mathfrak{R}, b \in \mathfrak{B})$.

Lemma 34. The smallest subspace of $\mathfrak{B}$ which contains $\mathfrak{S}$ and which is invariant under the adjoint representation of $\mathfrak{B}$, is $\mathfrak{B}$ itself.

Let $\mathfrak{M}$ be the smallest subspace satisfying the required conditions. For any $\alpha$, exp $\left(t\right.$ ad $\left.X_{\alpha}\right)=\sigma_{\alpha}(t)(t \in C)$ is a well defined automorphism of $\mathfrak{R}$. Clearly this can be extended uniquely to an automorphism of $\mathfrak{B}$. Let $G$ be the group generated by $\sigma_{\alpha}(t)$ for all roots $\alpha$ and all $t \in C$. Then it is known (see Chevalley [4]) that there exists a polynomial $f$ in $n$ variables with coefficients in $C$ such that $f \neq 0$ and if $X=\sum_{1 \leqq i \leqq n} t_{i} X_{i}\left(t_{i} \in C\right)$ and $f\left(t_{1}, \cdots, t_{n}\right)$ $\neq 0$ we can find a $\sigma \in G$ and an $H \in \mathfrak{h}$ such that $X=\sigma H$. It is clear that $\mathfrak{M}$ is invariant under $\sigma$. Therefore since $\mathfrak{S} \subset \mathfrak{M}$ and $\sigma$ is an automorphism of $\mathfrak{B}$, $X^{m}=\sigma H^{m} \in \mathfrak{M} \quad(m \geqq 1)$. Let $V_{m}$ denote the subspace of $\mathfrak{B}$ spanned by $S_{\left(i_{1}, \cdots, i_{m}\right)} X_{i_{1}} X_{i_{2}} \cdots X_{i_{m}}, 1 \leqq i_{1}, \cdots, i_{m} \leqq n ; m \geqq 0$. We claim that $V_{m} \subset \mathfrak{M}$. For $m=0$ this is immediate since $1 \in \mathfrak{S} \subset \mathfrak{M}$. Hence we may assume $m \geqq 1$. It will be sufficient to show that $V_{m} / V_{m} \cap \mathfrak{M}=\{0\}$. Suppose this is false. Then we can find a base $\omega_{\mu}, 1 \leqq \mu \leqq N$, for $V_{m} / V_{m} \cap \mathfrak{M}$. Let $\theta$ denote the natural mapping of $V_{m}$ on $V_{m} / V_{m} \cap \mathfrak{M}$. Then

$$
\theta\left(\underset{\left.i \cdots, i_{m}\right)}{S} X_{i_{1}} X_{i_{2}} \cdots X_{i_{m}}\right)=\sum_{1 \leqq \mu \leqq N} a_{i_{i_{1} i_{2}} \cdots i_{m}, \mu} \omega_{\mu} \quad\left(a_{i_{1} \cdots i_{m}, \mu} \in C\right) .
$$


Hence

$$
\theta\left(X^{m}\right)=\sum_{1 \leqq i_{1}, \cdots, i_{m} \leqq n} \sum_{1 \leqq \mu N N} t_{i_{1}} t_{i_{2}} \cdots t_{i_{m}} a_{i_{1} i_{2}} \cdots i_{m}, \mu \omega_{\mu} .
$$

But if $f\left(t_{1}, \cdots, t_{n}\right) \neq 0, X^{m}=\sigma H^{m} \in V_{m} \cap \mathfrak{M}$. Hence

$$
\sum_{1 \leqq i_{1}, \cdots, i_{m} \leqq n} a_{i_{1} i_{2} \cdots i_{m}, \mu} t_{i_{1}} t_{i_{2}} \cdots t_{i_{m}}=0, \quad 1 \leqq \mu \leqq N,
$$

whenever $f\left(t_{1}, \cdots, t_{n}\right) \neq 0$. Since $a_{i_{1} i_{2} \cdots i_{m}, \mu}$ are symmetric in $i_{1}, \cdots, i_{m}$ this implies that $a_{i_{1} i_{2} \cdots i_{m} \mu}=0$. But since $S_{\left(i_{1}, \cdots, i_{m}\right)} X_{i_{1}} X_{i_{2}} \cdots X_{i_{m}}$ span $V_{m}$, we must have $\theta\left(V_{m}\right)=\{0\}$ which contradicts our hypothesis. Hence $V_{m} \subset \mathfrak{M}$ and this being true for all $m, \mathfrak{B}=\sum_{m \geqq 0} V_{m} \subset \mathfrak{M}$.

Given any linear function $\lambda$ on $\mathfrak{h}$ we propose to associate with $\lambda$ a character $\chi_{\lambda}$ of $\mathfrak{B}$. Put $\rho=(1 / 2) \sum_{\alpha>0} \alpha$ and let $W$ be the Weyl group of $\&$ with respect to $\mathfrak{h}$. Let $\Delta$ denote the function $\prod_{\alpha>0}\left(\exp \left((-1)^{1 / 2} \alpha / 2\right)\right.$ $\left.-\exp \left(-(-1)^{1 / 2} \alpha / 2\right)\right)$ defined on $\mathfrak{h}$. Since every $s \in W$ induces a permutation $\alpha \rightarrow s \alpha$ of the roots of $\&$ it is clear that

$$
s \Delta=\prod_{\alpha>0}\left(\exp \left((-1)^{1 / 2} \frac{s \alpha}{2}\right)-\exp \left(-(-1)^{1 / 2} \frac{s \alpha}{2}\right)\right)= \pm \Delta .
$$

We say that $s$ is even or odd according as $s \Delta=+\Delta$ or $-\Delta$ and write $\epsilon(s)=1$ or -1 accordingly. It is known that the Weyl reflexion $s_{\alpha}$ with respect to a root $\alpha$ is odd (see Weyl [12]).

Let $\mathfrak{F}$ be the space of all linear functions on $\mathfrak{h}$. Then $\mathfrak{F}$ and $\mathfrak{h}$ are dual spaces and every $s \in W$ can be made to act on $\mathfrak{h}$ by duality so that

$$
\lambda(s H)=s^{-1} \lambda(H)
$$

for all $\lambda \in \mathfrak{F}$ and $H \in \mathfrak{h}$. Let $x_{1}, \cdots, x_{l}, t_{1}, \cdots, t_{l}$ be $2 l$ independent variables. For any $H \in \mathfrak{h}$ let $x(H)$ denote the linear form $\sum_{1 \leqq i \leqq l} c_{i} x_{i}, H=\sum_{1 \leqq i \leqq l} c_{i} H_{i}$. Also set $s x(H)=x\left(s^{-1} H\right)$ and $(s x)_{i}=s x\left(H_{i}\right), 1 \leqq i \leqq l$. For any $\lambda \in \mathfrak{F}$ we denote by $\lambda\left(H_{t}\right)$ the linear form $\sum_{1 \leqq i \leqq l} \lambda\left(H_{i}\right) t_{i}$ and write $\lambda\left(s H_{t}\right)=s^{-1} \lambda\left(H_{t}\right)$. Finally we put

$$
x\left(s H_{t}\right)=s^{-1} x\left(H_{t}\right)=\sum_{1 \leqq i \leqq l} t_{i} x\left(s H_{i}\right) .
$$

Consider the power series $\theta(x, t)$ in $x_{1}, \cdots, x_{l}, t_{1}, \cdots, t_{l}$ with coefficients in $C$ given by $\left({ }^{19}\right)$

$$
\theta(x, t)=\sum_{s \in W} \epsilon(s) \exp \left((-1)^{1 / 2} s x\left(H_{t}\right)\right) .
$$

Since $\epsilon\left(s_{\alpha} s\right)=-\epsilon(s)$,

$$
2 \theta(x, t)=\sum_{s \in W} \epsilon(s)\left\{\exp \left((-1)^{1 / 2} s x\left(H_{t}\right)\right)-\exp \left((-1)^{1 / 2} s_{\alpha} s x\left(H_{t}\right)\right)\right\} .
$$

(19) As usual exp $z$ stands for the power series $1+z+z^{2} / 2 !+z^{3} / 3 !+\cdots$. 
But $s_{\alpha} s x=s x-\left(s x\left(H_{\alpha}\right)\right) \alpha$. Hence

$$
\begin{aligned}
\exp \left((-1)^{1 / 2} s x\left(H_{t}\right)\right) & -\exp \left((-1)^{1 / 2} s_{\alpha} s x\left(H_{t}\right)\right) \\
& =\exp \left((-1)^{1 / 2} s x\left(H_{t}\right)\right)\left\{1-\exp \left(-(-1)^{1 / 2} s x\left(H_{\alpha}\right) \alpha\left(H_{t}\right)\right)\right\} .
\end{aligned}
$$

Now $1-\exp \left(-(-1)^{1 / 2} s x\left(H_{\alpha}\right) \alpha\left(H_{t}\right)\right)$ is divisible by $\alpha\left(H_{t}\right)$ in the ring of power series. Hence $\theta(x, t)$ is divisible by $\alpha\left(H_{t}\right)$. Similarly

$$
2 \theta(x, t)=\sum_{s \in W} \epsilon(s)\left\{\exp \left((-1)^{1 / 2} s x\left(H_{t}\right)\right)-\exp \left((-1)^{1 / 2} s s_{\alpha} x\left(H_{t}\right)\right)\right\} .
$$

Since $s s_{\alpha} x\left(H_{t}\right)=s_{\alpha} x\left(s^{-1} H_{t}\right)=x\left(s^{-1} H_{t}\right)-x\left(H_{\alpha}\right) \alpha\left(s^{-1} H_{t}\right)$, we get

$\exp \left((-1)^{1 / 2} s x\left(H_{t}\right)\right)-\exp \left((-1)^{1 / 2} s s_{\alpha} x\left(H_{t}\right)\right)$

$$
=\exp \left((-1)^{1 / 2} s x\left(H_{t}\right)\right)\left\{1-\exp \left(-(-1)^{1 / 2} x\left(H_{\alpha}\right) \alpha\left(s^{-1} H_{t}\right)\right)\right\} .
$$

Since $1-\exp \left(-(-1)^{1 / 2} x\left(H_{\alpha}\right) \alpha\left(s^{-1} H_{t}\right)\right)$ is divisible by $x\left(H_{\alpha}\right)$ it follows that $\theta(x, t)$ is divisible also by $x\left(H_{\alpha}\right)$. Now if $\alpha, \beta$ are roots greater than 0 and $\alpha \neq \beta, x\left(H_{\alpha}\right), \alpha\left(H_{t}\right), x\left(H_{\beta}\right), \beta\left(H_{t}\right)$ are all relatively prime. Since a power series ring over $C$ is a unique factorisation domain it follows that $\theta(x, t)$ is divis-

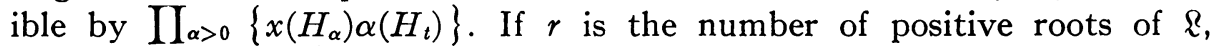
$\prod_{\alpha>0}\left\{x\left(H_{\alpha}\right) \alpha\left(H_{t}\right)\right\}$ is of degree $r$ in $(x)$ and $(t)$ each. Since each homogeneous term of $\theta(x, t)$ is clearly of the same degree in $(x)$ and $(t)$ the same must hold for the power series $\theta(x, t) / \prod_{\alpha>0}\left\{x\left(H_{\alpha}\right) \alpha\left(H_{t}\right)\right\}$. Now

$$
\frac{\exp \left((-1)^{1 / 2}\left(\alpha\left(H_{t}\right) / 2\right)\right)-\exp \left(-(-1)^{1 / 2}\left(\alpha\left(H_{t}\right) / 2\right)\right)}{\alpha\left(H_{t}\right)}
$$

is a unit in the power series ring since its constant term is $(-1)^{1 / 2} \neq 0$. Consider

$\frac{\theta(x, t)}{\prod_{\alpha>0}\left\{x\left(H_{\alpha}\right) \alpha\left(H_{t}\right)\right\}} \prod_{\alpha>0}\left\{\frac{\exp \left((-1)^{1 / 2}\left(\alpha\left(H_{t}\right) / 2\right)\right)-\exp \left(-(-1)^{1 / 2}\left(\alpha\left(H_{t}\right) / 2\right)\right)}{\alpha\left(H_{t}\right)}\right\}^{-1}$.

Since the last factor is a power series in $(t)$ only, it is obvious from the above remark that the product is a power series in $(x),(t)$ such that the coefficient of each power product $t_{1}^{m_{1}} t_{2}^{m_{2}} \cdots t_{l}^{m_{l}}$ is a polynomial in $(x)$ whose degree is less than or equal to $m_{1}+m_{2}+\cdots+m_{l}$. Put

$$
\begin{aligned}
\varphi(x, t)= & \prod_{\alpha>0} \rho\left(H_{\alpha}\right) \frac{\theta(x, t)}{\prod_{\alpha>0}\left\{x\left(H_{\alpha}\right) \alpha\left(H_{t}\right)\right\}} \\
& \cdot \prod_{\alpha>0}\left\{\frac{\exp \left((-1)^{1 / 2}\left(\alpha\left(H_{t}\right) / 2\right)\right)-\exp \left(-(-1)^{1 / 2}\left(\alpha\left(H_{t}\right) / 2\right)\right)}{\alpha\left(H_{t}\right)}\right\}^{-1} \\
= & \frac{\prod_{\alpha>0} \rho\left(H_{\alpha}\right)}{\prod_{\alpha>0} x\left(H_{\alpha}\right)} \frac{\theta(x, \dot{t})}{\Delta\left(H_{t}\right)}
\end{aligned}
$$


where $\Delta\left(H_{t}\right)$ is the power series $\prod_{\alpha>0}\left\{\exp \left((-1)^{1 / 2}\left(\alpha\left(H_{t}\right) / 2\right)\right)-\exp \left(-(-1)^{1 / 2}\right.\right.$ $\left.\left.\cdot\left(\alpha\left(H_{t}\right) / 2\right)\right)\right\}$. Then

$$
\varphi(x, t)=\sum_{m_{1}, \cdots, m_{l} \geqq 0} s_{m_{1}}, \cdots, m_{l}(x) t_{1}^{m_{1}} \cdots t_{l}^{m_{l}}
$$

where $s_{m_{1}, \ldots, m_{l}}(x) \in C[x]$.

Now put $x_{i}^{\prime}=x_{i}+\rho\left(H_{i}\right)$ and consider the power series

$$
\chi(x, t)=\sum_{m_{1}, \cdots, m_{l} \geq 0} s_{m_{1}}, \cdots, m_{l}\left(x^{\prime}\right) t_{1}^{m_{1} t_{2}^{m_{2}} \cdots t_{l}^{m_{l}}}
$$

where $s_{m_{1}, \cdots, m_{l}}\left(x^{\prime}\right)$ is obtained by replacing each $x_{i}$ in $s_{m_{1}, \cdots, m_{l}}(x)$ by $x_{i}^{\prime}$, $1 \leqq i \leqq l$. Then $\chi(x, t)$ is a power series in $(t)$ with coefficients in $C[x]$. Define a linear mapping $\chi_{x}$ of $\mathfrak{S}$ into $C[x]$ by the rule

$$
\chi_{x}\left(H_{1}^{m_{1}} H_{2}^{m_{2}} \cdots H_{l}^{m_{l}}\right)=\left(1 /\left((-1)^{1 / 2}\right)^{m_{1}+\cdots+m_{l}}\right) s_{m_{1}}, \cdots, m_{l}\left(x^{\prime}\right) .
$$

Since $H_{1}^{m_{1}} H_{2}^{m_{2}} \cdots H_{l}^{m_{l}}, m_{1}, \cdots, m_{l} \geqq 0$, form a base for $\mathfrak{W}$ this defines $\chi_{x}$ completely. Given any $\lambda \in \mathfrak{F}$ and $h \in \mathfrak{S}$ we denote by $\chi_{\lambda}(h)$ the values of the polynomial $\chi_{x}(h)$ at the point $x_{i}=\lambda\left(H_{i}\right), 1 \leqq i \leqq l$. In this way we get a linear mapping $\chi_{\lambda}$ of $\mathfrak{S}$ into $C$.

If $\Lambda \in \mathrm{P}$ and if $\pi_{\Lambda}$ is the corresponding finite-dimensional irreducible representation of $\mathfrak{B}$ with the highest weight $\Lambda$, then it is known (see Weyl [12]) that the degree $d_{\Lambda}$ of $\pi_{\Delta}$ is given by

$$
d_{\Lambda}=\frac{\prod_{\alpha>0} \Lambda^{\prime}\left(H_{\alpha}\right)}{\prod_{\alpha>0} \rho\left(H_{\alpha}\right)}
$$

where $\Lambda^{\prime}=\Lambda+\rho$. Moreover

$$
\operatorname{sp}\left(\exp (-1)^{1 / 2} \pi_{\Lambda}\left(H_{t}\right)\right)=d_{\Lambda} \sum_{m_{1}, \cdots, m_{l} \geqq 0} s_{m_{1}}, \cdots, m_{l}\left(\Lambda^{\prime}\right) t_{1}^{m_{1}} t_{2}^{m_{2}} \cdots t_{l}^{m_{l}}
$$

for any $t_{1}, \cdots, t_{l} \in C$ such that $\left|t_{1}\right|, \cdots,\left|t_{l}\right| \leqq \eta, \eta$ being a suitable real number greater than 0 . Here $H_{t}=\sum_{1 \leqq i \leqq l} t_{i} H_{i}$ and $s_{m_{1}}, \cdots, m_{l}\left(\Lambda^{\prime}\right)$ is the value of the polynomial $s_{m_{1}}, \cdots,_{m_{l}}(x)$ at the point $x_{i}=\Lambda^{\prime}\left(H_{i}\right), 1 \leqq i \leqq l$. Also $\exp (-1)^{1 / 2} \pi_{\Lambda}\left(H_{t}\right)$ denotes the usual exponential of the matrix $(-1)^{1 / 2} \pi_{\Lambda}\left(H_{t}\right)$. Hence by comparing coefficients of the two power series in (15) we get

$$
\frac{1}{d_{\Lambda}} \operatorname{sp} \pi_{\Lambda}\left(H_{1}^{m_{1}} H_{2}^{m_{2}} \cdots H_{l}^{m_{l}}\right)=\frac{1}{\left((-1)^{1 / 2}\right)^{m_{1}+\cdots+m_{l}}} s_{m_{1}, \cdots, m_{l}}\left(\Lambda^{\prime}\right)
$$

and therefore

$$
\chi_{\Lambda}(h)=\frac{1}{d_{\Lambda}} \operatorname{sp} \pi_{\Lambda}(h)
$$


in this case.

Let $\mathfrak{B}^{\prime}$ be the set of all elements in $\mathscr{B}$ which can be written as linear combination of elements of the form $\left[b_{1}, b_{2}\right]\left(b_{1}, b_{2} \in \mathfrak{B}\right)$.

Lemma 35. If $h \in \mathfrak{S} \cap \mathfrak{B}^{\prime}, \chi_{x}(h)=0$. Furthermore $\chi_{x}(1)=1$.

Let $\chi_{x}(h)=g(x)$. If $g(x) \neq 0$ we can find a $\Lambda \in P$ such that $g(\Lambda) \neq 0$. Consider the irreducible finite-dimensional representation $\pi_{\Delta}$ of $\mathfrak{B}$ whose highest weight is $\Lambda$. Then $\chi_{\Lambda}(h)=g(\Lambda) \neq 0$. On the other hand from (17)

$$
\chi_{\Delta}(h)=\frac{1}{d_{\Lambda}} \operatorname{sp} \pi_{\Lambda}(h)=0
$$

since $h \in \mathfrak{B}^{\prime}$. Thus we get a contradiction. Hence $\chi_{x}(h)=0$. We prove in exactly the same way that $\chi_{x}(1)-1=0$.

From Lemma 34 we know that $\mathfrak{B}=\mathfrak{B}^{\prime}+\mathfrak{S}$. Given any $z \in \mathfrak{B}$ let $z=b+h$ where $b \in \mathfrak{B}^{\prime}, h \in \mathfrak{S}$. If $b^{\prime} \in \mathfrak{B}^{\prime}, h^{\prime} \in \mathfrak{S}$ are two other elements such that $z=b^{\prime}+h^{\prime}$ then $h-h^{\prime} \in \mathfrak{S} \cap \mathfrak{B}^{\prime}$ and therefore by the above lemma $\chi_{x}\left(h-h^{\prime}\right)=0$. Hence it follows that $\chi_{x}(h)$ is uniquely determined by $z$ alone. We now extend $\chi_{x}$ to a linear mapping of $\mathfrak{B}$ into $C[x]$ by setting $\chi_{x}(z)=\chi_{x}(h)$. For any $\lambda \in \mathfrak{F}$ we define $\chi_{\lambda}$ on $\mathfrak{B}$ by $\chi_{\lambda}(z)=\left(\chi_{x}(z)\right)_{x_{i}=\lambda_{i}}, \lambda_{i}=\lambda\left(H_{i}\right), 1 \leqq i \leqq l$. It is clear that $\chi_{x}(b)=\chi_{\lambda}(b)=0$ for any $b \in \mathfrak{B}^{\prime}$.

Lemma 36. Given any $z \in Z$ there exists a unique element $f_{z}(x) \in C[x]$ such that $z-\beta^{-1}\left(f_{z}(x)\right) \in \mathfrak{P}$. Moreover the mapping $z \rightarrow f_{z}(z \in \mathbb{Z})$ is an isomorphism of 3 into $C[x]$. Finally, $f_{z}=\chi_{x}(z)$.

$\alpha_{1}, \cdots, \alpha_{r}$ being all the positive roots of $\mathfrak{R}$, put $X_{\alpha_{i}}=X_{i}, X_{-\alpha_{i}}=Y_{i}$, $1 \leqq i \leqq r$, and

$$
z(q, m, p)=Y_{1}^{q_{1}} Y_{2}^{q_{2}} \cdots Y_{r}^{q_{r}} H_{1}^{m_{1}} H_{2}^{m_{2}} \cdots H_{l}^{m_{l}} X_{1}^{p_{1}} \cdots X_{r}^{p_{r}}
$$

as in the proof of Theorem 2. Also we define ranks in $\mathfrak{B}$ exactly as there. Then $z(q, m, p)$ is of rank rank $p-\operatorname{rank} q$ where rank $p=\sum_{1 \leqq i \leqq r} p_{i} \alpha_{i}$, rank $q$ $=\sum_{1 \leqq i \leqq r} q_{i} \alpha_{i}$. It is clear that every $z \in \mathcal{Z}$ is of zero rank. Hence from Lemma $1, z$ is a linear combination of $z(q, m, p)$ with rank $q=$ rank $p$. But if rank $p>0, z(q, m, p) \in \mathfrak{B}$. Hence

$$
z \equiv \sum_{m} a(m) z(0, m, 0) \bmod \mathfrak{P} \quad\left(a(m) \in C^{\prime}\right) .
$$

This shows that there exists an $h \in \mathfrak{S}$ such that $z-h \in \mathfrak{P}$. Put $f(x)=\beta(h)$. Now if $g \in C[x]$ and $z-\beta^{-1}(g) \in \mathfrak{B}$ it follows that

$$
\beta^{-1}(g)-h \in \mathfrak{B} \cap \mathfrak{S}=\{0\}
$$

from Lemma 32. Hence $g-\beta(h)=g-f=0$. Therefore $f$ is unique.

Let $z_{1}, z_{2} \in \mathbb{B}$. Then if $h_{1}=\beta^{-1}\left(f_{z_{1}}\right), h_{2}=\beta^{-1}\left(f_{z_{2}}\right)$ 


$$
z_{1} z_{2}-h_{1} h_{2}=z_{2}\left(z_{1}-h_{1}\right)+h_{1}\left(z_{2}-h_{2}\right) \equiv 0 \bmod \mathfrak{B} .
$$

Hence it follows from the uniqueness established above that $f_{z_{1} z_{2}}=\beta\left(h_{1} h_{2}\right)$ $=f_{z_{1}} f_{z_{2}}$. Now let $z \in 乃, z \neq 0$. Then we can find a finite-dimensional irreducible representation $\pi_{\Lambda}$ of $\mathfrak{B}$ with the highest weight $\Lambda$ such that $\pi_{\Lambda}(z) \neq 0$ (see [9]). Since $\pi_{\Lambda}$ is irreducible it follows from Schur's lemma that $\pi_{\Lambda}(z)=c \pi_{\Lambda}(1)$ where $c \in C$. Let $\psi \neq 0$ be a vector in the representation space of $\pi_{\Delta}$ which belongs to the highest weight $\Lambda$. Then since $z-\beta^{-1}\left(f_{z}\right) \in \mathfrak{P}$,

$$
\pi_{\Lambda}\left(z-\beta^{-1}\left(f_{z}\right)\right) \psi=\left\{c-f_{z}(\Lambda)\right\} \psi=0 .
$$

Since $\psi \neq 0, f_{z}(\Lambda)=c \neq 0$. Therefore $f_{z} \neq 0$. This proves that $z \rightarrow f_{z}(z \in \mathbb{Z})$ is an isomorphism.

For any $z \in \Re$ put $g(x)=\chi_{x}(z)-f_{z}(x)$. If $g(x) \neq 0$ we can find a $\Lambda \in \mathrm{P}$ such that $g(\Lambda) \neq 0$. Then

$$
\chi_{\Lambda}(z)-f_{z}(\Lambda)=g(\Lambda) \neq 0 .
$$

Let $\psi \neq 0$ be a vector belonging to the highest weight $\Lambda$ of $\pi_{\Delta}$. Since $\pi_{\Lambda}$ is irreducible and $z \in \mathcal{B}, \pi_{\Lambda}(z)=c \pi_{\Lambda}(1)(c \in C)$ by Schur's lemma. Hence $c=\left(1 / d_{\Lambda}\right)$ sp $\pi_{\Lambda}(z)$.

Now

$$
\pi_{\Lambda}\left(\beta^{-1}\left(f_{z}\right)\right) \psi=f_{z}(\Lambda) \psi .
$$

Since $z-\beta^{-1}\left(f_{z}\right) \in \mathfrak{B}, \pi_{\Lambda}\left(z-\beta^{-1}\left(f_{z}\right)\right) \psi=0$. Hence

$$
\left\{\frac{1}{d_{\Lambda}} \operatorname{sp} \pi_{\Lambda}(z)-f_{z}(\Lambda)\right\} \psi=0 .
$$

Since $\psi \neq 0$,

$$
\frac{1}{d_{\Lambda}} \operatorname{sp} \pi_{\Lambda}(z)-f_{z}(\Lambda)=\chi_{\Lambda}(z)-f_{z}(\Lambda)=g(\Lambda)=0
$$

from (17). Thus we get a contradiction and therefore $\chi_{x}(z)=f_{z}(x)$.

We are now in a position to begin the proof of the following theorem.

THEOREM 5. $\chi_{\lambda}$ is a character for every linear function $\lambda$ on $\mathfrak{h}$. Given any homomorphism $\chi$ of 3 into $C$ such that $\chi(1)=1$ we can find a linear function $\lambda$ on $\mathfrak{h}$ such that $\chi(z)=\chi_{\lambda}(z)$ for all $z \in \mathcal{B}$. If $\lambda_{1}, \lambda_{2}$ are two linear functions on $\mathfrak{h}$ then $\chi_{\lambda_{1}}=\chi_{\lambda_{2}}$ if and only if $\lambda_{2}+\rho=s\left(\lambda_{1}+\rho\right)$ for some $s \in W$.

Corollary. Every homomorphism $\chi$ of $B$ into $C$ such that $\chi(1)=1$ can be extended uniquely to a character of $\mathfrak{B}$. Every character of $\mathfrak{B}$ is of the form $\chi_{\lambda}$ where $\lambda$ is a linear function on $\mathfrak{h}$.

This is an immediate consequence of Theorem 5 and Lemma 31.

The first assertion of the theorem follows directly from Lemmas 35 and 
36 and the definition of $\chi_{\lambda}$. In order to prove the rest we first need some lemmas.

Lemma 37. Let $C\{x\}$ be the power series ring in $x_{1}, \cdots, x_{l}$ with coefficients in $C$ and let $\mathfrak{N}$ be the ideal in $C\{x\}$ generated by $x_{1}, \cdots, x_{l}$. Given any $f(x)$ $\in C[x]$ and an integer $N \geqq 1$ we can find a finite number of linear forms $\lambda_{j}(x)$, $1 \leqq j \leqq r$, such that

$$
\sum_{1 \leqq j \leqq r} c_{j} \exp \lambda_{j}(x) \equiv f(x) \bmod \mathfrak{R}^{N} \cdot \quad\left(c_{j} \in C\right) .
$$

If $N=1$ we may clearly take $r=1$ and $\lambda_{1}(x)=0$. Hence we may assume $N \geqq 2$ and use induction on $N$. Notice that

$$
x_{i}-\left(\exp x_{i}\right)+1 \in \mathfrak{N}^{2}, \quad 1 \leqq i \leqq l .
$$

By induction hypothesis we can find linear forms $\lambda_{j}(x), 1 \leqq j \leqq r$, and $c_{j} \in C$ such that

$$
\sum_{1 \leqq i \leqq r} c_{j} \exp \lambda_{j}(x) \equiv f(x) \bmod \mathfrak{N}^{N-1}
$$

Hence

$$
f(x) \equiv \sum_{1 \leqq j \leqq r} c_{j} \exp \lambda_{j}(x)+g(x) \bmod \mathfrak{R}^{N}
$$

where $g(x)$ is a form of degree $N-1$. Let $G$ denote the power series obtained by replacing each $x_{i}$ by exp $\left(x_{i}\right)-1$ in $g(x)$. Clearly $g(x) \equiv G \bmod \mathfrak{N}^{N}$. Hence

$$
f(x) \equiv \sum_{1 \leqq j \leqq r} c_{j} \exp \lambda_{j}(x)+G \bmod \mathfrak{N}^{N} .
$$

The expression on the right is of the required type since $G$ is clearly a linear combination of exponentials of linear forms. The lemma is therefore proved.

Notice that if $\lambda(x)$ is any given linear form we can find an $H \in \mathfrak{h}$ such that $\lambda(x)=(-1)^{1 / 2} x(H)$. Hence by the above lemma

$$
f(x) \equiv \sum_{1 \leqq j \leqq r} c_{j} \exp (-1)^{1 / 2} x\left(H_{(j)}\right) \bmod \mathfrak{R}^{N}
$$

for suitable $c_{j} \in C$ and $H_{(j)} \in \mathfrak{h}$.

For any $s \in W$ the mapping $x_{i} \rightarrow(s x)_{i}=s x\left(H_{i}\right), 1 \leqq i \leqq l$, can be extended uniquely to an automorphism of $C\{x\}$. We denote this extension again by $s$. Let $f(x)$ be a polynomial such that $s f(x)=f(x)$ for all $s \in W$. Put

$$
g(x)=f(x) \prod_{\alpha>0} x\left(H_{\alpha}\right) .
$$

It is known (see Weyl [12]) that $\prod_{\alpha>0} s x\left(H_{\alpha}\right)=\epsilon(s) \prod_{\alpha>0} x\left(H_{\alpha}\right)$. Hence

$$
s g(x)=\epsilon(s) g(x) .
$$

Now by Lemma 37, 


$$
g(x) \equiv \sum_{1 \leqq j \leqq r} c_{j} \exp \left((-1)^{1 / 2} x\left(H_{(j)}\right)\right) \bmod \mathfrak{R}^{N} \quad\left(c_{j} \in C, H_{(j)} \in \mathfrak{h}\right) .
$$

Since $\mathfrak{N}$ is invariant under $W$ it follows that

$$
\begin{aligned}
g(x) & =\frac{1}{h} \sum_{: \in W} \epsilon(s)(s g(x)) \\
& \equiv \frac{1}{h} \sum_{1 \leqq j \leqq r} c_{j} \sum_{s \in W} \epsilon(s) \exp \left((-1)^{1 / 2} s x\left(H_{(j)}\right)\right) \bmod \mathfrak{R}^{N}
\end{aligned}
$$

where $h$ is the order of $W$. We prove exactly as before that the power series on the right-hand side is divisible by $\prod_{\alpha>0} x\left(H_{\alpha}\right)$. Hence

$$
f(x) \equiv \sum_{1 \leqq j \leqq r} c_{j}^{\prime}\left\{\frac{\sum_{s \in W} \in(s) \exp (-1)^{1 / 2} s x\left(H_{(j)}\right)}{\prod_{\alpha>0} x\left(H_{\alpha}\right)}\right\} \bmod \mathfrak{\Re}^{M}\left(c_{j}^{\prime} \in C\right)
$$

where $N-M$ is the degree of the form $\prod_{\alpha>0} x\left(H_{\alpha}\right)$. Since $N$ could be chosen arbitrarily large the same is true of $M$. Let us choose $M$ greater than the degree of $f(x)$. Then it follows immediately that $f(x)$ is a finite linear combination of the coefficients of the power series

$$
\varphi_{0}(x, t)=\frac{\sum_{s} \in W \epsilon(s) \exp \left((-1)^{1 / 2} s x\left(H_{t}\right)\right)}{\prod_{\alpha>0} x\left(H_{\alpha}\right)}
$$

if we regard it as a power series in $\left(t_{1}, \cdots, t_{l}\right)$ with coefficients in $C[x]$. Now put $x_{i}^{\prime}=x_{i}+\rho\left(H_{i}\right), 1 \leqq i \leqq l$, as before. From (11) and (12) it is clear that the coefficients of $\chi(x, t)$ are linear combinations of the coefficients of $\varphi_{0}\left(x^{\prime}, t\right)$ and conversely. Hence $f\left(x^{\prime}\right)$ is a linear combination of $\chi_{x}\left(H_{1}^{m_{1}} H_{2}^{m_{2}} \cdots H_{l}^{m_{l}}\right), m_{1}, \cdots, m_{l} \geqq 0$. Thus we have proved the following lemma.

LEMMA 38. If $f(x)$ is a polynomial in $C[x]$ such that $s f(x)=f(x)$ for all $s \in W$ then $f\left(x^{\prime}\right)=\chi_{x}(b)$ for some $b \in \mathfrak{B}$.

Let $\Re$ be the set of all elements in $C[x]$ of the form $\chi_{x}(z)(z \in Z)$. We shall show that $\Re$ coincides with the set of all elements of the form $\chi_{x}(b)(b \in \mathfrak{B})$.

Lemma 39. Let $\mathfrak{B}^{\prime}=[\mathfrak{B}, \mathfrak{B}]$ as before. Then $\mathfrak{B}=\mathfrak{B}^{\prime}+\mathfrak{Z}$.

Let $V=\mathfrak{B}+\mathfrak{B}^{\prime}$. Suppose $V \neq \mathfrak{B}$. Then we can find a subspace $U$ of $\mathfrak{B}$ such that $\mathfrak{B}=V+U, V \cap U=\{0\}, U \neq 0$. Clearly $\operatorname{dim} U$ is either finite or countable. Let $e_{j}, 1 \leqq j<N$, be a base for $U$, where $N$ is either a positive integer or $\infty . \lambda$ being any linear function on $\mathfrak{h}$ we define a linear function $\chi$ on $\mathfrak{B}$ as follows:

$$
\chi(z)=\chi_{\lambda}(z), \quad z \in V, \quad \chi\left(e_{1}\right)=\chi_{\lambda}\left(e_{1}\right)+1, \quad \chi\left(e_{j}\right)=\chi_{\lambda}\left(e_{j}\right), \quad j \geqq 2 .
$$

Since $\chi$ coincides with $\chi_{\lambda}$ on $V$ it is clear that $\chi$ is a character. However 
$\chi \neq \chi_{\lambda}$ since $\chi\left(e_{1}\right) \neq \chi_{\lambda}\left(e_{1}\right)$. But this contradicts Lemma 31. Hence $V=\mathfrak{B}$.

COROLlaRY. $\Re$ coincides with the set of all polynomials of the form $\chi_{x}(b)$ $(b \in \mathfrak{B})$.

For, by the above lemma, $b=z+b^{\prime}$ when $z \in \mathfrak{B}, b^{\prime} \in \mathfrak{B}^{\prime}$. Hence $\chi_{x}(b)$ $=\chi_{x}(z) \in \Re$.

Lemma 40. Let $U$ be an indeterminate. The coefficients of the polynomial

$$
\mathrm{II}_{s \in W^{*}}\left(U-s x\left(H_{t}\right)-s \rho\left(H_{t}\right)\right)
$$

regarded as a polynomial in $U$ lie in $\Re[t]$.

Consider

$$
g(U, x, t)=\prod_{s \in W}\left(U-s x\left(H_{t}\right)\right) .
$$

Clearly this polynomial is invariant under the substitution $x_{i} \rightarrow(s x)_{i}, 1 \leqq i \leqq l$. Hence it follows from Lemma 38 and the above corollary that the coefficients of $g\left(U, x^{\prime}, t\right)$ regarded as a polynomial in $U$ and $(t)$ lie in $\Re$. This proves the lemma.

Corollary. $C[x]$ is integrally dependent on $\Re$.

On making the substitution $t_{j}=0,1 \leqq j \leqq l, i \neq j, t_{i}=1$, we find from the above lemma that $x_{i}^{\prime}$ and therefore $x_{i}$ is integrally dependent on $\Re$. These being true for every $i, 1 \leqq i \leqq l$, the assertion follows.

We can now prove the second part of Theorem 5. $\chi$ being any homomorphism of $\mathbb{Z}$ into $C$ such that $\chi(1)=1$, put $\chi^{\prime}\left(\chi_{x}(z)\right)=\chi(z) \quad(z \in \mathbb{Z})$. Then $\chi^{\prime}$ is a homomorphism of $\Re$ into $C$ and $\chi^{\prime}(1)=1$. Since $C[x]$ is integrally dependent on $\Re$ it follows from well known results in algebra that every homomorphism of $\Re$ into $C$ can be extended to a homomorphism of $C[x]$ into $C$. We denote such an extension of $\chi^{\prime}$ again by $\chi^{\prime}$. Let $\lambda$ be the linear function on $\mathfrak{h}$ such that $\chi^{\prime}\left(x_{i}\right)=\lambda\left(H_{i}\right), 1 \leqq i \leqq l$. Then $\chi(z)=\chi^{\prime}\left(\chi_{2}(z)\right)=\chi_{\lambda}(z)$. This proves the second part of the theorem.

For the proof of the last part we proceed as follows. Let $C\{t\}$ and $C[t]$ denote the power series and the polynomial rings respectively in $(t)$ with coefficients in $C$. We denote by $\partial / \partial t_{i}$ the uniquely determined $C$-derivation of $C\{t\}$ such that $\partial t_{j} / \partial t_{i}=\delta_{i j}, 1 \leqq i, j \leqq l$.

Lemma 41. Let $\lambda_{1}, \cdots, \lambda_{r}$ be any $r$ distinct linear functions on $\mathfrak{h}$. Then the elements $\exp \left(\lambda_{i}\left(H_{t}\right)\right) \in C\{t\}, 1 \leqq i \leqq r$, are linearly independent over $C[t]$, that is,

$$
\sum_{1 \leqq j \leqq r} f_{j}(t) \exp \left(\lambda_{j}\left(H_{t}\right)\right)=0, \quad f_{j}(t) \in C[t]
$$

implies $f_{j}(t)=0,1 \leqq j \leqq r$. 
Suppose the assertion is false. Let $m$ be the least integer such that there exist polynomials $f_{j}(t), 1 \leqq j \leqq r$, not all zero and all of degree less than or equal to $m$ such that $\sum_{1 \leqq j \leqq r} f_{j}(t) \exp \left(\lambda_{j}\left(H_{t}\right)\right)=0$. Let $s$ be the number of polynomials among these which are not zero and which have degree $m$. Clearly $s \geqq 1$. We choose that particular set of $f_{j}$ for which $s$ has the least possible value. Then

$$
\sum_{1 \leqq j \leqq r} f_{j}(t) \exp \left(\lambda_{j}\left(H_{t}\right)\right)=0
$$

and by differentiation

$$
\sum_{1 \leqq j \leqq r}\left\{\lambda_{j}\left(H_{i}\right) f_{j}(t)+\frac{\partial f_{j}(t)}{\partial t_{i}}\right\} \exp \left(\lambda_{j}\left(H_{t}\right)\right)=0, \quad 1 \leqq i \leqq l .
$$

We may assume that $f_{1}(t) \neq 0$ and its degree is $m$. Since $\lambda_{1}, \cdots, \lambda_{r}$ are all distinct we can find $c_{i} \in C, 1 \leqq i \leqq l$, such that if $H=\sum_{1 \leqq i \leqq l} c_{i} H_{i}, \lambda_{1}(H)$ $\not \lambda_{j}(H), 2 \leqq j \leqq r$. Put

$$
g_{i}(t)=\left\{\lambda_{j}(H)-\lambda_{1}(H)\right\} f_{j}(t)+\sum_{1 \leqq i \leqq l} c_{i} \frac{\partial f_{j}(t)}{\partial t_{i}}, \quad 1 \leqq j \leqq r .
$$

Then from (18) and (19)

$$
\sum_{1 \leqq i \leqq r} g_{j}(t) \exp \left\{\lambda_{j}\left(H_{t}\right)\right\}=0 .
$$

Not all $g_{j}(t)$ are zero. For otherwise

$$
\left\{\lambda_{j}(H)-\lambda_{1}(H)\right\} f_{j}(t)=-\sum_{1 \leqq i \leqq l} c_{i} \frac{\partial f_{j}(t)}{\partial t_{i}}, \quad 1 \leqq j \leqq r .
$$

If $j \neq 1, \lambda_{j}(H) \neq \lambda_{1}(H)$ and (20) is impossible unless $f_{j}(t)=0$. But then $f_{1}(t) \exp \left(\lambda_{1}\left(H_{t}\right)\right)=0$. Since $C\{t\}$ is an integral domain and since exp $\left(\lambda_{1}\left(H_{t}\right)\right)$ $\neq 0, f_{1}(t)=0$. Therefore $f_{j}(t)=0,1 \leqq j \leqq r$, which contradicts our hypothesis. Moreover it is clear that the degree of $g_{j}(t)$ is not greater than that of $f_{j}(t)$. Also $g_{1}(t)$ is of degree less than or equal to $m-1$. Hence at most $s-1$ polynomials among $g_{j}(t)$ are of degree $m$. But this contradicts the definition of $s$. Hence the lemma is proved.

Using the same notation as before, we consider the power series

$$
\varphi_{0}(x, t)=\frac{\prod_{s} \in W \epsilon(s) \exp \left((-1)^{1 / 2} s x\left(H_{t}\right)\right)}{\prod_{\alpha>0} x\left(H_{\alpha}\right)}
$$

as a power series in $(t)$ with coefficients in $C[x]$. Given a power series $\xi(x, t)$ in $(t)$ with coefficients in $C[x]$ and any $\lambda \in \mathfrak{F}$ we denote by $\xi(\lambda, t)$ the series in $C\{t\}$ obtained by substituting $x_{i}=\lambda\left(H_{i}\right), i \leqq i \leqq l$, in the coefficients of $\xi(x, t)$.

LEMma 42. Let $\lambda_{1}, \lambda_{2}$ be two linear functions on $\mathfrak{h}$ such that $c_{1} \varphi_{0}\left(\lambda_{1}, t\right)$ 
$=c_{2} \varphi_{0}\left(\lambda_{2}, t\right)$ where $c_{1}, c_{2}$ are complex numbers not both zero. Then $\lambda_{2}=s \lambda_{1}$ for some $s \in W$.

Let $\partial / \partial x_{i}$ denote differentiation with respect to $x_{i}$ in $C[x]$ and for any power series $\theta(x, t)$ with coefficients in $C[x]$ let $\partial \theta(x, t) / \partial x_{i}$ denote the series obtained by differentiating the coefficients of $\theta(x, t)$ with respect to $x_{i}$. Then

$$
\varphi_{0}(x, t) \prod_{\alpha>0} x\left(H_{\alpha}\right)=\sum_{s \in W} \epsilon(s) \exp \left((-1)^{1 / 2} s x\left(H_{t}\right)\right) .
$$

Differentiating $r$ times we get

$$
\begin{aligned}
\frac{\partial^{r}}{\partial x_{i_{1}} \partial x_{i_{2}} \cdots \partial x_{i_{r}}} & \left\{\varphi_{0}(x, t) \prod_{\alpha>0} x\left(H_{\alpha}\right)\right\} \\
= & \sum_{s \in W} \epsilon(s)\left((-1)^{1 / 2}\right)^{r} \frac{\partial s x\left(H_{t}\right)}{\partial x_{i_{1}}} \cdots \frac{\partial s x\left(H_{t}\right)}{\partial x_{i_{r}}} \exp \left((-1)^{1 / 2} s x\left(H_{t}\right)\right) .
\end{aligned}
$$

Put

$$
\begin{aligned}
f_{i_{1} i_{2}}^{(s)} \ldots i_{r} & (t)=\left((-1)^{1 / 2}\right)^{r} \frac{\partial s x\left(H_{t}\right)}{\partial x_{i_{1}}} \cdots \frac{\partial s x\left(H_{t}\right)}{\partial x_{i_{r}}} \\
g_{i_{1} i_{2}} \cdots i_{r} & (x)=\frac{\partial^{r}}{\partial x_{i_{1}} \partial x_{i_{2}} \cdots \partial x_{i_{r}}} \prod_{\alpha>0} x\left(H_{\alpha}\right) .
\end{aligned}
$$

$f^{(s)}(t)=1$ and $g(x)=\prod_{\alpha>0} x\left(H_{\alpha}\right)$. Since $g(x) \neq 0$, it is clear that there exists an $r \geqq 0$ such that $g_{i_{1} i_{2} \cdots i_{r}}\left(\lambda_{1}\right) \neq 0$ for some $i_{1}, i_{2}, \cdots, i_{r}$. (We define $g_{i_{1} \cdots i_{r}}(x)$ $=g(x)$ if $r=0$. Similarly for $f_{i_{1}}^{(s)} \ldots i_{r}(t)$.) Choose the least such $r$. Then

$$
\begin{aligned}
\sum_{s \in W} \epsilon(s) f_{i_{1} i_{2} \cdots i_{r}}^{(s)}(t) \exp \left((-1)^{1 / 2} s \lambda_{1}\left(H_{t}\right)\right) & \\
= & {\left[\frac{\partial^{r}}{\partial x_{i_{1}} \cdots \partial x_{i_{r}}}\left\{\varphi_{0}(x, t) \prod_{\alpha>0} x\left(H_{\alpha}\right)\right\}\right]_{x_{i}=\lambda_{1}\left(H_{i}\right)} } \\
= & g_{i_{1} i_{2}} \cdots i_{r}\left(\lambda_{1}\right) \varphi_{0}\left(\lambda_{1}, t\right) .
\end{aligned}
$$

Since $g_{i_{1} i_{2} \ldots i_{r}}\left(\lambda_{1}\right) \neq 0$,

$$
\varphi_{0}\left(\lambda_{1}, t\right)=\frac{\sum_{s} \in W \epsilon(s) f_{i_{1} i_{2} \cdots i_{r}}^{(s)}(t) \exp \left((-1)^{1 / 2} s \lambda_{1}\left(H_{t}\right)\right)}{g_{i_{1} i_{2}} \cdots i_{r}\left(\lambda_{1}\right)} .
$$

Similarly

$$
\varphi_{0}\left(\lambda_{2}, t\right)=\frac{\sum{ }_{s} \in W \epsilon(s) f_{j_{1} \cdots j_{r^{\prime}}}^{(s)}(t) \exp \left((-1)^{1 / 2} s \lambda_{2}\left(H_{t}\right)\right)}{g_{j_{1} \cdots j_{r^{\prime}}}\left(\lambda_{2}\right)}
$$

where $g_{j_{1} j_{2} \cdots j_{r}}\left(\lambda_{2}\right) \neq 0$. It is clear from the definition of $\varphi_{0}(x, t)$ that 


$$
\varphi_{0}(x, t)=\varphi(x, t) \frac{\Delta\left(H_{t}\right)}{\prod_{\alpha>0 \rho}\left(H_{\alpha}\right)},
$$

where $\varphi(x, t)$ is defined by (10). Since the coefficient of 1 in $\varphi(x, t)$ is $\chi_{x}(1)=1$, it follows that $\varphi\left(\lambda_{1}, t\right) \neq 0$ and therefore $\varphi_{0}\left(\lambda_{1}, t\right) \neq 0$. Similarly $\varphi_{0}\left(\lambda_{2}, t\right) \neq 0$. On the other hand $c_{1} \varphi_{0}\left(\lambda_{1}, t\right)-c_{2} \varphi_{0}\left(\lambda_{2}, t\right)=0$ where $c_{1}, c_{2}$ are not both zero. Hence from Lemma 41, $s_{1} \lambda_{1}=s_{2} \lambda_{2}$ for some $s_{1}, s_{2} \in W$. Therefore $\lambda_{2}=s \lambda_{1}$ where $s=s_{2}^{-1} s_{1}$.

Now we can complete the proof of Theorem 5. Let $\chi(x, t)$ be defined as in (13). Then $\chi_{\lambda_{1}}=\chi_{\lambda_{2}}$ implies that $\chi\left(\lambda_{1}, t\right)=\chi\left(\lambda_{2}, t\right)$, and therefore $\varphi_{0}\left(\lambda_{1}+\rho, t\right)$ $=\varphi_{0}\left(\lambda_{2}+\rho, t\right)$. Hence from Lemma $42, \lambda_{2}+\rho=s\left(\lambda_{1}+\rho\right)$ for some $s \in W$, and Theorem 5 is proved completely.

Notice that in the definition of the series $\chi(x, t)$ on p. 71 we made use of the positive roots rather than of the negative roots. We could have equally well considered the series

$$
\chi^{-}(x, t)=\frac{\sum_{s \in W \epsilon}(s) \exp \left((-1)^{1 / 2} s x^{\prime \prime}\left(H_{t}\right)\right)}{\prod_{\alpha<0} x^{\prime \prime}\left(H_{\alpha}\right) \Delta^{\prime}\left(H_{t}\right)} \prod_{\alpha<0} \rho^{\prime}\left(H_{\alpha}\right),
$$

$x_{i}^{\prime \prime}=x_{i}+\rho^{\prime}\left(H_{i}\right), 1 \leqq i \leqq l, \rho^{\prime}=2^{-1} \sum_{\alpha<0} \alpha=-\rho, \Delta^{\prime}=\prod_{\alpha<0}\left(e^{(-1)^{1 / 2} \alpha / 2}-e^{-(-1)^{1 / 2} \alpha / 2}\right)$. Then

$$
\chi^{-}(-x, t)=\overline{\chi(x, t)}=\chi(x,-t)
$$

where $\overline{\chi(x, t)}$ is obtained from $\chi(x, t)$ by changing the coefficients to their complex conjugates. Let $\left((-1)^{1 / 2}\right)^{m_{1}+\cdots+{ }^{m} l} \chi_{x}^{-}\left(H_{1}^{m_{1}} \cdots H_{l}^{m_{l}}\right)$ denote the coefficient of $t_{1}^{m_{1}} \ldots t_{l}^{m_{l}}$ in $\chi^{-}(x, t)$. We define $\chi_{x}^{-}(h)(h \in \mathfrak{S})$ by linearity and extend $\chi_{x}^{-}$ to a linear mapping of $B$ into $C[x]$ exactly as in the case of $\chi_{x}$ by setting $\chi_{x}^{-}(z)$ $=\chi_{x}^{-}(h)$ where $z=h+b\left(h \in \mathfrak{S}, b \in \mathfrak{B}^{\prime}=[\mathfrak{B}, \mathfrak{B}], z \in \mathfrak{B}\right)$. Let $\mathfrak{N}=\sum_{\alpha<0} \mathfrak{B} X_{\alpha}$. Then corresponding to Lemma 36 , we prove that $z-\beta^{-1}\left(\chi_{x}^{-}(z)\right) \in \mathfrak{N}(z \in \Re)$. Let $\varphi$ denote the linear mapping $X \rightarrow-X(X \in \mathfrak{R})$ of $\mathfrak{R}$ into itself. Since $\varphi\left(\left[X, X^{\prime}\right]\right)=\left[X^{\prime}, X\right]=\left[\varphi\left(X^{\prime}\right), \varphi(X)\right]\left(X, X^{\prime} \in \mathbb{R}\right), \varphi$ can be extended uniquely to an anti-automorphism of $\mathfrak{B}$. Clearly $\varphi(\mathfrak{Z})=\mathfrak{Z}$. Now for any $z \in \mathbb{Z}$,

$$
z-\beta^{-1}\left(\chi_{x}(z)\right) \in \mathfrak{P} \text {. }
$$

Hence

$$
z-\beta^{-1}\left(\chi_{x}(z)\right)=\sum_{\alpha>0} z_{\alpha} X_{\alpha}
$$

where $z_{\alpha}$ is of rank $-\alpha$. Hence

$$
\varphi(z)-\varphi\left(\beta^{-1}\left(\chi_{x}(z)\right)=\sum_{\alpha>0} \varphi\left(X_{\alpha}\right) \varphi\left(z_{\alpha}\right) .\right.
$$

But

$$
\left[H, \varphi\left(z_{\alpha}\right)\right]=-\left[\varphi(H), \varphi\left(z_{\alpha}\right)\right]=\varphi\left(\left[H, z_{\alpha}\right]\right)=-\alpha(H) \varphi\left(z_{\alpha}\right)
$$


for any $H \in \mathfrak{h}$. Hence $\varphi\left(z_{\alpha}\right)$ is of rank $-\alpha$ and therefore lies in $\mathfrak{N}$. Hence

$$
\overline{\chi_{x}}(\varphi(z))=\beta\left(\varphi\left(\beta^{-1}\left(\chi_{x}(z)\right)\right)\right)=\chi_{-x}(z)
$$

where the polynomial $\chi_{-x}(z)$ is obtained from $\chi_{x}(z)$ by the substitution $x_{i} \rightarrow-x_{i}, 1 \leqq i \leqq l$. For any $\lambda \in \mathfrak{F}$, we denote by $\chi_{\lambda}^{-}$the linear function on $\mathfrak{B}$ such that $\chi_{\lambda}^{-}(z)=f(\lambda)$ where $f(x)=\chi_{x}^{-}(z)(z \in \mathfrak{B})$. We prove exactly as in the case of $\chi_{\lambda}$ that $\chi_{\lambda}^{-}$is a character. Therefore from Theorem $5, \chi_{\lambda}^{-}=\chi_{\mu}$ for some $\mu \in \mathfrak{F}$. But then

$$
\begin{aligned}
\chi^{-}(\lambda, t) & =\sum_{m_{1}, \cdots, m_{l} \geqq 0}\left((-1)^{1 / 2}\right)^{m_{1}+\cdots+m_{l}} \chi_{\lambda}^{-}\left(H_{1}^{m_{1}} \cdots H_{l}^{m_{l}}\right) t_{1}^{m_{1}} \cdots t_{l}^{m_{l}} \\
& =\sum_{m_{1}, \cdots, m_{l} \geqq 0}\left((-1)^{1 / 2}\right)^{m_{1}+\cdots+m_{l}} \chi_{\mu}\left(H_{1}^{m_{1}} \cdots H_{l}^{m_{l}}\right) t_{1}^{m_{1}} \cdots t_{l}^{m_{l}}=\chi(\mu, t) .
\end{aligned}
$$

Hence from Lemma $42, \lambda-\rho=s(\mu+\rho)$ for some $s \in W$. Changing $\lambda$ to $-\lambda$, we get the result that $\chi_{-\lambda}^{-}=\chi_{\mu}$ if and only if

$$
-(\lambda+\rho)=s(\mu+\rho) .
$$

Let $\pi$ be a representation of $\mathscr{B}$ and $\chi$ a character of $\mathfrak{B}$. We shall say that $\pi$ has the character $\chi$ if $\pi(z-\chi(z))=0$ for all $z \in \mathcal{B}$. From the corollary to Theorem 5 it is clear that $\pi$ has a uniquely determined character provided $\pi(z)$ is a multiple of $\pi(1)$ for every $z \in 3$.

We shall now apply the above results to the situation considered in Theorem 4. From now on we adhere strictly to the notation of Part II. In particular $\mathfrak{R}, \mathfrak{g}, \mathfrak{g}^{+}, \mathfrak{R}_{K}, \mathfrak{B}, \mathfrak{A}$, and $\mathfrak{X}$ have the same meaning as there. $\mathfrak{A}^{+}$is the algebra generated by $\mathrm{g}^{+}$and 1 . The isomorphisms $\gamma$ and $\gamma^{+}$of $\mathfrak{R}_{K}$ with $\mathfrak{g}$ and $\mathfrak{g}^{+}$respectively have been defined on p. 53. Then $\gamma\left(\mathfrak{h}_{K}\right)$ is a Cartan subalgebra of $\mathfrak{g}$ and every linear function $\lambda$ on $\mathfrak{h}_{K}$ can also be regarded as a linear function on $\gamma\left(\mathfrak{h}_{K}\right)$ and conversely by the rule $\lambda(\gamma(H))=\lambda(H)\left(H \in \mathfrak{h}_{K}\right)$. Under this correspondence roots of $\mathfrak{R}_{K}$ with respect to $\mathfrak{h}_{K}$ are also roots of $\mathfrak{g}$ with respect to $\gamma\left(\mathfrak{h}_{K}\right)$. Hence the Weyl group $W$ of $\mathfrak{R}_{K}$ is also the Weyl group of $\mathfrak{g}$. Similar remarks hold for $\mathfrak{g}^{+}$. Since $\mathfrak{l}$ is the direct sum of $\mathfrak{g}$ and $\mathfrak{g}^{+}, \mathfrak{h}$ $=\gamma\left(\mathfrak{h}_{K}\right)+\gamma^{+}\left(\mathfrak{h}_{K}\right)$ is a Cartan subalgebra of $\mathfrak{R}$. Every linear function $\nu$ on $\mathfrak{h}$ can be regarded as a pair $(\lambda, \mu)$ of linear functions on $\mathfrak{h}_{K}$ by the rule

$$
\nu(H)=\lambda\left(H_{1}\right)+\mu\left(H_{2}\right)
$$

where $H=\gamma\left(H_{1}\right)+\gamma\left(H_{2}\right)\left(H \in \mathfrak{h}, H_{1}, H_{2} \in \mathfrak{h}_{K}\right)$. It is easily seen that the roots of $\mathbb{R}$ are exactly those pairs which are of the form $(\alpha, 0)$ or $(0, \alpha)$ where $\alpha$ is a root of $\mathfrak{R}_{K}$. Let $\left\{\alpha_{1}, \cdots, \alpha_{l}\right\}$ be a fundamental system of roots of $\mathfrak{R}_{K}$ with respect to $\mathfrak{h}_{K}$. Then the set $\left(\alpha_{i}, 0\right)\left(0, \alpha_{i}\right), 1 \leqq i \leqq l$, is a fundamental system of roots of $\mathfrak{R}$ with respect to $\mathfrak{h}$ and therefore the Weyl group of $\mathfrak{R}$ is the direct product $W \times W$.

Let $(\lambda, \mu)$ be a pair of linear functions on $\mathfrak{h}_{K}$. This pair defines a linear 
function on $\mathfrak{h}$ and therefore a character of $\mathfrak{B}$. We denote this character by $\chi_{\lambda, \mu}$. Let $X_{\alpha}$ be a root element in $\mathfrak{R}_{K}$ corresponding to the root $\alpha$. Put $\left[X_{\alpha}, X_{-\alpha}\right]=H_{\alpha}, H_{i}=H_{\alpha_{i}}, 1 \leqq i \leqq l$. Let $x_{1}, \cdots, x_{l}, y_{1}, \cdots, y_{l}, t_{1}, \cdots, t_{l}$, $u_{1}, \cdots, u_{l}$ be independent variables. Then corresponding to (12) we have to consider the power series

$$
\begin{aligned}
& \chi(x, y, t, u) \\
(24) & =\left\{\prod_{\alpha>0} \rho\left(H_{\alpha}\right)\right\}^{2} \frac{\sum_{s, \sigma \in W} \epsilon(s) \epsilon(\sigma) \exp \left((-1)^{1 / 2} s x^{\prime}\left(H_{t}\right)+(-1)^{1 / 2} \sigma y^{\prime}\left(H_{u}\right)\right)}{\prod_{\alpha>0} x^{\prime}\left(H_{\alpha}\right) \prod_{\alpha>0} y^{\prime}\left(H_{\alpha}\right) \Delta\left(H_{t}\right) \Delta\left(H_{u}\right)} \\
& =\chi(x, t) \chi(y, t)
\end{aligned}
$$

where the notation is analogous to that of pp. 71-72. $x_{i}^{\prime}=x_{i}+\rho\left(H_{i}\right), y_{i}^{\prime}=y_{i}$ $+\rho\left(H_{i}\right)$, and $\rho=2^{-1} \sum_{\alpha>0} \alpha$. We define the linear mapping $\chi_{x, y}$ of $\mathfrak{B}$ into $C[x, y]$ exactly as on p. 72 . Similarly let $\chi_{x}$ and $\chi_{y}$ denote the corresponding linear mappings of $\mathfrak{X}$ into $C[x]$ and $C[y]$ respectively. Whenever necessary we shall also regard $\chi_{x}$ and $\chi_{y}$ as linear mappings of $\mathfrak{A}$ and $\mathfrak{A}^{+}$so that

$$
\chi_{x}(\gamma(\omega))=\chi_{x}(\omega), \quad \chi_{y}\left(\gamma^{+}(\omega)\right)=\chi_{y}(\omega) \quad(\omega \in \mathfrak{X})
$$

where the isomorphisms $\gamma$ and $\gamma^{+}$have now been extended (uniquely) on $\mathfrak{X}$. It follows from (24) that

$$
\begin{aligned}
& \text { (25) } \chi_{x, y}\left(\gamma\left(H_{1}^{m_{1}} \cdots H_{l}^{m_{l}}\right) \gamma^{+}\left(H_{1}^{n_{1}} \cdots H_{l}^{n_{l}}\right)\right)=\chi_{x}\left(H_{1}^{m_{1}} \cdots H_{l}^{m l}\right) \chi_{y}\left(H_{1}^{n_{1}} \cdots H_{l}^{n_{l}}\right) \text {, } \\
& m_{i}, n_{i} \geqq 0 .
\end{aligned}
$$

Let $\chi$ denote the linear mapping of $\mathscr{B}$ into $C[x, y]$ such that $\chi\left(a a^{+}\right)$ $=\chi_{x}(a) \chi_{y}\left(a^{+}\right)\left(a \in \mathfrak{A}, a^{+} \in \mathfrak{A}^{+}\right)$. Then clearly $\chi\left(\left[b_{1}, b_{2}\right]\right)=0$ for any $b_{1}, b_{2} \in \mathfrak{B}$. Also $\chi$ coincides with $\chi_{x, y}$ on the algebra $\mathfrak{S}$ generated by $\mathfrak{h}$ and 1 . Hence from Lemma 34, $\chi=\chi_{x, y}$ and therefore $\chi_{x, y}\left(a a^{+}\right)=\chi_{x}(a) \chi_{y}\left(a^{+}\right)\left(a \in \mathfrak{A}, a^{+} \in \mathfrak{A}+\right)$. We express this relation symbolically in the form $\chi_{x, y}=\chi_{x} \times \chi_{y}$. It is clear that $\chi_{\lambda, \mu}\left(a a^{+}\right)=\chi_{\lambda}(a) \chi_{\mu}\left(a^{+}\right)$. Hence we again write $\chi_{\lambda, \mu}=\chi_{\lambda} \times \chi_{\mu}$.

Let $\mathfrak{Z}$, $\mathfrak{E}$, and $\mathfrak{E}^{+}$be the centers of $\mathfrak{B}, \mathfrak{A}$, and $\mathfrak{A}^{+}$respectively. Clearly $\chi_{x, y}(z)=\chi_{x}(z)$ if $z \in \mathbb{C}$ and $\chi_{x, y}\left(z^{+}\right)=\chi_{y}\left(z^{+}\right)$if $z^{+} \in \mathfrak{C}^{+}$. Hence $\chi_{x, y}(\mathbb{Z})$ contains $\chi_{x}(\mathfrak{S})$ and $\chi_{y}(\mathfrak{G})$. Let $\Omega$ be the set of all elements $\chi_{x, y}(z)(z \in Z)$ such that $\left.{ }^{20}\right)$ $\nu(z)\left(1 \times 1^{*}\right)=0$.

Lemma 43. Let $\Lambda_{i}, 0 \leqq i \leqq N$, be all the distinct weights of $\mathfrak{D}_{\Lambda_{0}}$. Then the coefficients of the polynomial

$F(x, y, t)=\prod_{0 \leqq i \leqq N} \prod_{s, \sigma \in W}\left(\sigma y\left(H_{t}\right)+\sigma \rho\left(H_{t}\right)+s x\left(H_{t}\right)+s \rho\left(H_{t}\right)-\Lambda_{i}\left(H_{t}\right)\right)$

regarded as a polynomial in $(t)$ are all in $\Omega$.

Exactly as in the proof of Lemma 40 , we show that these coefficients lie

${ }^{(20)}$ We recall that $\nu$ is the representation of $\mathscr{B}$ on $\mathfrak{Q} X \mathfrak{X}^{*}$ (see p. 59). $\Lambda_{0}$ is a fixed dominant integral function on $\mathfrak{h}_{K}$ and $\mathfrak{X}^{*}=\mathfrak{A} / \mathfrak{A} \mathfrak{Y}_{\Lambda_{0}}$. 
in the ring generated by $\chi_{x}(\mathbb{S})$ and $\chi_{y}\left(\mathfrak{S}^{+}\right)$and therefore in $\chi_{x, y}(\mathbb{Z})$. Therefore we can write

$$
F(x, y, t)=\sum_{m_{1}, \cdots, m_{l} \geqq 0} \chi_{x, y}\left(z\left(m_{1}, \cdots, m_{l}\right)\right) t_{1}^{m_{1}} t_{2}^{m_{2}} \cdots t_{l}^{m_{l}}, z\left(m_{1}, \cdots, m_{l}\right) \in 乃 .
$$

We have to show that $\nu\left(z\left(m_{1}, \cdots, m_{l}\right)\right)\left(1 \times 1^{*}\right)=0, m_{1}, \cdots, m_{l} \geqq 0$. Suppose this is false. Then for some $z$ among $z\left(m_{1}, \cdots, m_{l}\right)$

$$
\nu(z)\left(1 \times 1^{*}\right) \neq 0 \text {. }
$$

Choose $\omega_{i}, 1 \leqq i \leqq p$, in $\mathfrak{X}$ such that $\omega_{i}^{*}$ form a base for $\mathfrak{X}^{*}$. Then

$$
\nu(z)\left(1 \times 1^{*}\right)=\sum_{1 \leqq i \leqq p} a_{i} \times \omega_{i}^{*}, \quad a_{i} \in \mathfrak{R} .
$$

Since $\nu(z)\left(1 \times 1^{*}\right) \neq 0$ we can find an $a_{i}$, say $a_{1}$, such that $a_{1} \neq 0$. By Theorem 1 of [9] there exists a finite-dimensional representation $\pi$ of $\mathfrak{A}$ such that $\pi\left(a_{1}\right)$ $\neq 0$. Clearly we may choose $\pi$ so that it is irreducible.

Let $\theta$ denote the linear mapping of $\mathrm{g}^{+}$on $\mathrm{g}$ given by $\theta\left(\gamma^{+}(X)\right)=-\gamma(X)$ $\left(X \in \mathfrak{R}_{K}\right)$. It is easily verified that

$$
\theta\left(\left[Z_{1}^{+}, Z_{2}^{+}\right]=-\left[\theta\left(Z_{1}^{+}\right), \theta\left(Z_{2}^{+}\right)\right] \quad\left(Z_{1}^{+}, Z_{2}^{+} \in \mathfrak{g}^{+}\right) .\right.
$$

Hence $\theta$ can be extended uniquely to an anti-isomorphism of $\mathfrak{A}^{+}$onto $\mathfrak{A}$. Let $\sigma$ denote the representation of $\mathfrak{B}$ on $\pi(\mathfrak{A})$ defined by

$$
\sigma\left(a^{\prime}\right) \pi(a)=\pi\left(a^{\prime} a\right), \quad \sigma(X) \pi(a)=\pi([X, a]) \quad\left(X \in \mathfrak{R}_{K} ; a^{\prime}, a \in \mathfrak{I}\right) .
$$

Then

$$
\begin{aligned}
\sigma\left(Z^{+}\right) \pi(a) & =\sigma\left(Z^{+}\right) \sigma(a) \pi(1)=\sigma(a) \sigma\left(Z^{+}\right) \pi(1) \\
& =\pi(a) \pi\left(\theta\left(Z^{+}\right)\right) \quad\left(Z^{+} \in \mathfrak{g}^{+}, a \in \mathfrak{U}\right)
\end{aligned}
$$

since $\left[Z^{+}, a\right]=0$ and $Z^{+}=X-\gamma(X)$ if $Z^{+}=\gamma^{+}(X)\left(X \in \mathbb{R}_{K}\right)$. From this it follows easily that

$$
\begin{aligned}
\sigma\left(a^{+}\right) \pi(a) & =\pi(a) \pi\left(\theta\left(a^{+}\right)\right) \\
\sigma\left(a^{\prime}\right) \pi(a) & =\pi\left(a^{\prime}\right) \pi(a) .
\end{aligned} \quad\left(a^{+} \in \mathfrak{N}^{+} ; a, a^{\prime} \in \mathfrak{A}\right) .
$$

Since $\pi$ is irreducible, $\pi(\mathfrak{A})$ is a simple algebra. Therefore it has no ideals other than $\{0\}$ and itself. Hence $\sigma$ is an irreducible representation.

Let $P_{K}$ be the set of all dominant integral functions on $\mathfrak{h}_{K}$. For any $\Lambda_{1}, \Lambda_{2} \in \mathrm{P}_{K}$ let $\varphi_{1}, \varphi_{2}$ be two irreducible representations of $\mathfrak{R}_{K}$ belonging to $\mathfrak{D}_{\Lambda_{1}}$ and $\mathfrak{D}_{\Lambda_{2}}$ respectively. Extend $\varphi_{1}, \varphi_{2}$ to representations of $\mathbb{R}$ by setting $\varphi_{1}\left(Z^{+}\right)=0$ for all $Z^{+} \in \mathrm{g}^{+}$and $\varphi_{2}(Z)=0$ for all $Z \in \mathrm{g}$. The representation $\varphi_{1}+\varphi_{2}$ of $\mathcal{R}$ is then known to be irreducible. We denote by $\mathfrak{D}_{\Lambda_{1}, \Lambda_{2}}$ the class of all representations of $\mathfrak{R}$ equivalent to $\varphi_{1}+\varphi_{2}$. It is known that $\mathfrak{D}_{\Lambda_{1}, \Lambda_{2}} \neq \mathfrak{D}_{\Lambda_{1}^{\prime}, \Lambda_{2}^{\prime}}$ unless $\Lambda_{1}=\Lambda_{1}^{\prime}, \Lambda_{2} \neq \Lambda_{2}^{\prime}$, and every irreducible finite-dimensional representation 
of $\mathfrak{R}$ is contained in some $\mathfrak{D}_{\Lambda_{1}, \Lambda_{2}}$. Hence $\sigma \in \mathfrak{D}_{\lambda_{, \mu}}$ for some $\lambda, \mu \in \mathrm{P}_{K}$. By Schur's lemma, $\sigma(z)=\chi(z) \sigma(1)(z \in \mathbb{Z})$ where $z \rightarrow \chi(z)$ is a homomorphism of $\mathbb{Z}$ into $C$. Hence by Theorem $5, \chi$ can be extended uniquely to a character of $\mathfrak{B}$. From the theory of finite-dimensional representations it is known that this character is $\chi_{\lambda, \mu}$. Finally we note that the zero representation of $\mathfrak{R}_{K}$ occurs in $\sigma$ since $\sigma(X) \pi(1)=0\left(X \in \mathbb{R}_{K}\right)$ and $\pi(1) \neq 0$. Hence again it follows from known theory that

$$
\chi(\mu, t)=\overline{\chi(\lambda, t)}
$$

where $\overline{\chi(\lambda, t)}$ is obtained by changing the coefficients of the series $\chi(\lambda, t)$ (regarded as a series in $(t))$ to their complex conjugates. Applying Lemma 42, we get immediately

$$
\mu+\rho=-s(\lambda+\rho) \quad(s \in W) .
$$

Now consider the representation $\pi^{+}$of $\mathfrak{B}$ induced on $\pi(\mathfrak{A}) \times \mathfrak{X}^{*}$. It is given by

$$
\begin{aligned}
& \pi^{+}(X)\left(\pi(a) \times \omega^{*}\right)=\pi([X, a]) \times \omega^{*}+\pi(a) \times(X \omega)^{*}, \\
& \pi^{+}(Z)\left(\pi(a) \times \omega^{*}\right)=\pi(Z) \pi(a) \times \omega^{*} \quad\left(X \in \mathfrak{R}_{K}, \omega \in \mathfrak{X}, a \in \mathfrak{A}, Z \in \mathfrak{g}\right) .
\end{aligned}
$$

Let $\mathfrak{N}_{\pi}$ be the kernel of $\pi$ in $\mathfrak{A}$. It is easily seen that if $\xi$ is the natural mapping of $\mathfrak{A} \times \mathfrak{X}^{*}$ on $\mathfrak{A} \times \mathfrak{X}^{*} / \mathfrak{N}_{\pi} \times \mathfrak{X}^{*}$ then $\pi^{+}$is equivalent to the representation of $\mathfrak{B}$ induced by $\nu$ on $\mathfrak{A} \times \mathfrak{X}^{*} / \mathfrak{N}_{\pi} \times \mathfrak{X}^{*}$ under the isomorphism $\pi(a) \times \omega^{*} \leftrightarrow \xi\left(a \times \omega^{*}\right)$ $\left(a \in \mathfrak{A}, \omega^{*} \in \mathfrak{X}^{*}\right)$ of $\pi(\mathfrak{U}) \times \mathfrak{X}^{*}$ with $\mathfrak{A} \times \mathfrak{X}^{*} / \mathfrak{N}_{\pi} \times \mathfrak{X}^{*}$. We may therefore identify $\pi(\mathfrak{A}) \times \mathfrak{X}^{*}$ and $\mathfrak{A} \times \mathfrak{X}^{*} / \mathfrak{N}_{\pi} \times \mathfrak{X}^{*}$ under this isomorphism. Since $\pi\left(a_{1}\right) \neq 0$, $\pi^{+}(z)\left(\pi(1) \times 1^{*}\right)=\sum_{1 \leqq i \leqq N} \pi\left(a_{i}\right) \times \omega^{*} \neq 0$. Since $\pi(\mathfrak{A}) \times \mathfrak{X}^{*}$ is finite-dimensional it is fully reducible under $\pi^{+}(\mathscr{B})$. Then it follows easily that we can find a maximal invariant $\left.{ }^{21}\right)$ subspace $\mathfrak{M}_{*}$ of $\pi(\mathfrak{H}) \times \mathfrak{X}^{*}$ such that $\pi^{+}(z)\left(\pi(1) \times 1^{*}\right)$ $\notin \mathfrak{M}_{*}$. Let $\pi^{\prime}$ be the irreducible representation of $\mathscr{B}$ induced on $\left(\pi(\mathfrak{H}) \times \mathfrak{X}^{*}\right) / \mathfrak{M}_{*}$. Let $\pi^{\prime} \in \mathfrak{D}_{\lambda^{\prime}, \mu^{\prime}}\left(\lambda^{\prime}, \mu^{\prime} \in \mathrm{P}_{K}\right)$. Since the representation of $\mathfrak{B}$ on $\mathfrak{X}^{*}$ is of the type $\mathfrak{D}_{0, \Lambda_{0}}$ it follows from known results that $\lambda^{\prime}=\lambda$ and $\mu^{\prime}=\mu+\Lambda_{i}$ where $\Lambda_{i}$ is some weight of $\mathfrak{D}_{\Lambda_{0}}$. Since $\mu+\rho=-s(\lambda+\rho)(s \in W)$,

$$
s \lambda^{\prime}\left(H_{t}\right)+s \rho\left(H_{t}\right)+\mu^{\prime}\left(H_{t}\right)+\rho\left(H_{t}\right)-\Lambda_{i}\left(H_{t}\right)=0 .
$$

Therefore $F\left(\lambda^{\prime}, \mu^{\prime}, t\right)=0$. On the other hand

$$
F(x, y, t)=\sum_{m_{1}, \cdots, m_{l} \geqq 0} \chi_{x, y}\left(z\left(m_{1}, \cdots, m_{l}\right)\right) t_{1}^{m_{1}} t_{2}^{m_{2}} \cdots t_{l}^{m_{l}} .
$$

Hence

$$
F\left(\lambda^{\prime}, \mu^{\prime}, t\right)=\sum_{m_{1}, \cdots, m_{l} \geqq 0} \chi_{\lambda^{\prime}, \mu^{\prime}}\left(z\left(m_{1}, \cdots, m_{l}\right)\right) t_{1}^{m_{1}} \cdots t_{l}^{m_{l}}
$$

${ }^{(1)} \mathfrak{M}_{*}$ is maximal independently of the property of not containing $\pi^{+}(z)\left(\pi(1) \times 1^{*}\right)$. 
Since $\pi^{+}(z)\left(\pi(1) \times 1^{*}\right) \notin \mathfrak{M}_{*}, \pi^{\prime}(z) \neq 0$. Therefore as $z \in \mathbb{Z}, \pi^{\prime}(z)=\chi_{\lambda^{\prime}, \mu^{\prime}}(z) \pi^{\prime}(1)$ $\neq 0$. Hence $\chi_{\lambda^{\prime} \mu^{\prime}}(z) \neq 0$. Since $z$ is one of the elements $z\left(m_{1}, \cdots, m_{l}\right)$, it follows that $F\left(\lambda^{\prime}, \mu^{\prime}, t\right) \neq 0$. Thus we get a contradiction. This proves the lemma.

Let $\pi$ be any representation of $\mathfrak{B}$ with the character $\chi$ such that $\mathfrak{D}_{\Lambda_{0}}$ occurs in $\pi$. Let $f(x, y) \in \Omega$. Then $f(x, y)=\chi_{x, y}(z)$ where $z \in \bigcap$ and $\nu(z)\left(1 \times 1^{*}\right)=0$. Let $V$ be the representation space of $\pi$. By hypothesis $V_{\Lambda_{0}} \neq 0$. Choose $\psi$ $\in V_{\Lambda_{0}, \Lambda_{0}}, \psi \neq 0$, and let $\mathfrak{M}$ be the left ideal in $\mathfrak{B}$ consisting of all $b \in \mathfrak{B}$ such that $\pi(b) \psi=0$. Then $\mathfrak{M} \supset \mathfrak{Y Y} \mathfrak{\Lambda}_{\Lambda_{0}}$. Since $\nu(z)\left(1 \times 1^{*}\right)=0, z \in \mathfrak{U Y Y} \Lambda_{\Lambda_{0}}$ from Lemma 27. Hence $z \in \mathfrak{M}$ and $\pi(z)=0$. Therefore $f(\lambda, \mu)=\chi_{\lambda, \mu}(z)=0$. Applying this to the polynomial $F(x, y, t)$ of the above lemma we find that

$$
s \lambda\left(H_{t}\right)+s \rho\left(H_{t}\right)+\sigma \mu\left(H_{t}\right)+\sigma \rho\left(H_{t}\right)-\Lambda_{i}\left(H_{t}\right)=0
$$

for some $s, \sigma \in W$ and $i, 0 \leqq i \leqq N$. Now for every $\Lambda_{i}$ and $\tau \in W, \tau \Lambda_{i}$ is also a weight of $\mathfrak{D}_{\Lambda_{0}}$. Hence

$$
\lambda\left(H_{t}\right)+\rho\left(H_{t}\right)+\tau \mu\left(H_{t}\right)+\tau \rho\left(H_{t}\right)-\Lambda_{j}\left(H_{t}\right)=0
$$

for some $\tau \in W$ and some $j, 0 \leqq j \leqq N$. Put $\tau(\mu+\rho)=-\left(\mu^{\prime}+\rho\right)$. Then $\lambda-\mu^{\prime}=\Lambda_{j}$ and $\chi_{\mu}=\chi_{-\mu^{\prime}}^{-}$from (23). Thus we have proved the following theorem.

THEOREM 6. Let $\pi$ be a representation of $\mathfrak{B}$ with the character $\chi$ such that $\mathfrak{D}_{\Lambda_{0}}$ occurs in $\pi\left(\Lambda_{0} \in \mathrm{P}_{K}\right)$. Then $\chi=\chi_{\lambda} \times \chi_{-\mu}^{-}$where $\lambda-\mu$ is an integral function on $\mathfrak{h}_{K}$ which is a weight of $\mathfrak{D}_{\Lambda_{0}}$.

COROLlaRY. Let $\pi$ be any representation of $\mathfrak{B}$ with the character $\chi=\chi_{\lambda}$ $\times \chi_{-\mu}^{-}$. For any $\Lambda \in \mathrm{P}_{K}, \mathfrak{D}_{\Lambda}$ cannot occur in $\pi$ unless $(\lambda+\rho)-\sigma(\mu+\rho)$ is a weight of $\mathfrak{D}_{\Lambda}$ for some $\sigma \in W$.

Let $\mathfrak{l}_{0}$ and $\mathfrak{R}_{K, 0}$ be the Lie algebras over the field $R$ of real numbers as defined on p. 52. Henceforward we suppose that $\Omega_{K, 0}$ is compact, that is, the quadratic form $\mathrm{sp}(\operatorname{ad} X)^{2}\left(X \in \mathfrak{R}_{K, 0}\right)$ is negative definite. Let $\mathfrak{B}_{0}$ be the universal enveloping algebra of $\mathfrak{R}_{0}$. Then $\mathfrak{B}_{0}$ is an algebra over $R$ and $\mathfrak{B}$ can be regarded, in the obvious way, as the extension of $\mathfrak{B}_{0}$ over $C$. Then every $b \in \mathfrak{B}$ can be written uniquely in the form $b=b_{1}+(-1)^{1 / 2} b_{2}\left(b_{1}, b_{2} \in \mathfrak{B}_{0}\right)$. Let $\mathfrak{Z}_{0}$ be the center of $\mathfrak{B}_{0}$. It is easy to show (see [9, p. 914]) that the elements of $\mathfrak{Z}_{0}$ span over $C$ the center $\mathfrak{B}$ of $\mathfrak{B}$. Let $\varphi$ denote the linear mapping of $\mathfrak{R}_{0}$ into itself given by $\varphi(X)=-X\left(X \in \mathfrak{R}_{0}\right) . \varphi$ can be extended uniquely to an antiautomorphism of $\mathfrak{B}_{0}$. We now extend $\varphi$ on $\mathfrak{B}$ as follows. If $b=b_{1}+(-1)^{1 / 2} b_{2}$ $\left(b_{1}, b_{2} \in \mathfrak{B}\right)$ we put $\varphi(b)=\varphi\left(b_{1}\right)-(-1)^{1 / 2} \varphi\left(b_{2}\right)$. Let $\chi$ be any character of $\mathfrak{B}$. We shall denote by $\chi^{*}$ the linear function on $\mathfrak{B}$ defined as follows:

$$
\chi^{*}(b)=\overline{\chi(\varphi(b))}
$$

where the bar denotes complex conjugate. It is easy to verify that $\chi^{*}$ is also a character.

Let $\mathfrak{h}_{K, 0}$ be a maximal abelian subalgebra of $\mathfrak{l}_{K, 0}$ and $\mathfrak{h}_{K}$ the complexifica- 
tion of $\mathfrak{h}_{K, 0}$. Then $\mathfrak{h}_{K}$ is a Cartan subalgebra. If $\alpha$ is any root of $\mathfrak{R}_{K}$ with respect to $\mathfrak{h}_{K}$ and $H_{\alpha}$ the corresponding element in $\mathfrak{h}_{K}$ then it is known that $(-1)^{1 / 2} H_{\alpha} \in \mathfrak{h}_{K, 0}$. In particular if $\left\{\alpha_{1}, \cdots, \alpha_{l}\right\}$ is a fundamental set of roots and $H_{\alpha_{i}}=H_{i}, 1 \leqq i \leqq l, H_{i}^{\prime}=(-1)^{1 / 2} H_{i} \in \mathfrak{h}_{K, 0}$. Moreover $\varphi(\gamma(X))=-\gamma^{+}(X)$, $\varphi\left(\gamma^{+}(X)\right)=-\gamma(X)$ for any $X \in \mathfrak{R}_{K, 0}$. Hence

$$
\begin{aligned}
& \chi\left(\varphi\left(\gamma\left(H_{1}^{\prime m_{1}} \cdots H_{l}^{\prime m_{l}}\right) \gamma^{+}\left(H_{1}^{\prime n_{1}} \cdots H_{l}^{\prime{ }^{n} l}\right)\right)\right) \\
& =(-1)^{m_{1}+\cdots+m_{l}+n_{1}+\cdots+n_{l}} \chi\left(\gamma^{+}\left(H_{1}^{\prime m_{1}} \cdots H_{l}^{\prime m_{l}}\right) \gamma\left(H_{1}^{\prime n_{1}} \cdots H_{l}{ }^{\prime n_{l}}\right)\right) \text {, }
\end{aligned}
$$

since $\varphi$ is clearly an anti-automorphism of $\mathfrak{B}$ over $R$. Hence

$$
\begin{aligned}
& \chi^{*}\left(\gamma^{+}\left(H_{1}^{\prime m_{1}} \cdots H_{l}^{\prime m_{l}}\right) \gamma\left(H_{1}^{n_{1}} \cdots H_{l}^{\prime n_{l}}\right)\right)
\end{aligned}
$$

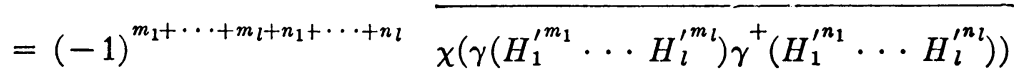

$$
\begin{aligned}
& \left(m_{1}, \cdots, m_{l}, n_{1}, \cdots, n_{l} \geqq 0\right) \text {. }
\end{aligned}
$$

For any linear function $\lambda$ on $\mathfrak{h}_{K}$ define $\bar{\lambda}$ by $\bar{\lambda}\left(H_{i}\right)=\overline{\lambda\left(H_{i}\right)}, 1 \leqq i \leqq l$. Further if $\xi$ is any polynomial (or series) we denote by $\bar{\xi}$ the polynomial (or series) obtained by changing the coefficients of $\xi$ to their complex conjugates. Suppose $\chi=\chi_{\lambda} \times \chi_{\mu}$ and $\chi^{*}=\chi_{\lambda} * \times \chi_{\mu}{ }^{*}$ where $\lambda, \mu, \lambda^{*}, \mu^{*}$ are linear functions on $\mathfrak{h}_{K}$. Then from (27) it follows that

$$
\left.\overline{\chi\left(\lambda^{*}, \mu^{*}, t, u\right.}\right)=\chi(\mu, \lambda,-t,-u)
$$

in the notation of (24). But, from (21), $\chi(\mu, \lambda,-t,-u)=\overline{\chi(\bar{\mu}, \bar{\lambda}, t, u)}$. Hence

$$
\chi\left(\lambda^{*}, \mu^{*}, t, u\right)=\chi(\bar{\mu}, \bar{\lambda}, t, u) .
$$

Therefore from Lemma $42, \chi^{*}=\chi_{\lambda *} \times \chi_{\mu *}=\chi_{\bar{\mu}} \times \chi_{\bar{\lambda}}$. Thus we have the following lemma.

Lemma 44. Let $\chi=\chi_{\lambda} \times \chi_{\mu}$ be a character of $\mathfrak{B}$. Then $\chi^{*}=\chi_{\bar{\mu}} \times \chi_{\bar{\lambda}}$.

COROLlaRY. $\chi=\chi^{*}$ if and only if $\chi=\chi_{\lambda} \times \chi_{\lambda}$ for some linear function $\lambda$ on $\mathfrak{h}_{K}$.

Part IV. Representations of a complex semisimple Lie group in a Hilbert space. So far we have considered only abstract representations of the Lie algebra $\mathfrak{l}_{0}$. Now we come to the representations of the corresponding group in a Hilbert space. Let $G$ be the simply connected Lie group whose Lie algebra is $\mathfrak{R}_{0}$, the latter being defined as in Part II. We assume that $\mathfrak{R}_{K, 0}$ is compact and $\mathfrak{h}_{K, 0}$ is a maximal abelian subalgebra of $\mathfrak{l}_{K, 0}$ and $\mathfrak{h}_{K}=\mathfrak{h}_{K, 0}$ $+(-1)^{1 / 2} \mathfrak{h}_{K, 0}$. We shall adhere closely to the notation of Parts II and III.

Let $V$ be a Hilbert space and $\pi$ a mapping which associates to each $g \in G$ a bounded operator $\pi(g)$ on $V$ such that $\pi\left(g_{1} g_{2}\right)=\pi\left(g_{1}\right) \pi\left(g_{2}\right)\left(g_{1}, g_{2} \in G\right)$ and $\pi(1)=I$, where 1 is the unit element of $G$ and $I$ is the unit operator on $V$. 
$\pi$ is called a representation of $G$ on $V$ if for every $\psi \in V$ the mapping $g \rightarrow \pi(g) \psi$ $(g \in G)$ is continuous. Moreover it is called an irreducible representation if there exists no closed subspace other than $V$ and $\{0\}$ which is invariant under $\pi(g)$ for all $g \in G$.

Since $G$ is a Lie group it is also an analytic manifold. Let $C_{c}^{\infty}(G)$ denote the class of all complex-valued functions on $G$ which are infinitely differentiable everywhere and which vanish outside a compact set. For any $X \in \mathcal{R}_{0}$ and $f \in C_{c}^{\infty}(G)$ we define $-X f \in C_{c}^{\infty}(G)$ by the rule

$$
(X f)(g)=\left\{\frac{d}{d t} f((\exp (-t X)) g)\right\}_{t=0} .
$$

It is easily verified that if $X_{1}, X_{2}, X_{3} \in \Omega_{0}$ and $\left[X_{1}, X_{2}\right]=X_{3}$ then

$$
X_{1}\left(X_{2} f\right)-X_{2}\left(X_{1} f\right)=X_{3} f \text {. }
$$

Thus we get a representation of $\Omega_{0}$ (and therefore by linearity a representation of $\mathfrak{R})$ on $C_{c}^{\infty}(G)$. We extend this uniquely to a representation of $\mathfrak{B}$. Then for any $z \in \mathscr{B}$ and $f \in C_{c}^{\infty}(G)$ the function $-z f$ is well defined. Let $V_{1}$ be the subspace spanned by all elements $\varphi \in V$ of the form

$$
\varphi=\int_{G} f(g) \pi(g) \psi d g
$$

where $\psi \in V$ and $f \in C_{c}^{\infty}(G)$ and $d g$ is the left invariant Haar measure on $G$. It has been shown by Gårding [8] that $V_{1}$ is dense in $V$ and

$$
\operatorname{Lim}_{t \rightarrow 0} \frac{1}{t}\{\pi(\exp t X) \varphi-\varphi\}=\int_{G}-X f(g) \pi(g) \psi d g \quad\left(X \in \mathfrak{R}_{0}\right)
$$

where $\varphi$ is given by (29). Let $\pi(X)$ denote the operator on $V_{1}$ defined by

$$
\pi(X) \varphi=\operatorname{Lim}_{t \rightarrow 0} \frac{1}{t}\{\pi(\exp t X) \varphi-\varphi\} \quad\left(X \in \mathfrak{\Omega}_{0}, \varphi \in V_{1}, t \in R\right) .
$$

Then $\pi(X)$ maps $V_{1}$ into itself and it is obvious from (30) that $\pi\left(\left[X_{1}, X_{2}\right]\right)$ $=\pi\left(X_{1}\right) \pi\left(X_{2}\right)-\pi\left(X_{2}\right) \pi\left(X_{1}\right)\left(X_{1}, X_{2} \in \Omega_{0}\right)$. Hence we get a representation of $\mathfrak{R}_{0}$ and therefore of $\mathfrak{B}$ on $V_{1}$ which we shall denote again by $\pi$. We shall call $V_{1}$ the Garding subspace of $V$.

Let $B$ be the center of $B$ and $\chi$ a character. We shall say that the representation $\pi$ of $G$ on $V$ has the character $\chi$ if $\pi(z) \varphi=\chi(z) \varphi$ for every $\varphi \in V_{1}$ and $z \in 3$. It is known that if $\pi(G)$ is an irreducible unitary representation then $\pi(z)(z \in Z)$ is a multiple of the unit operator on $V_{1}$. Hence $\pi$ has a uniquely determined character in this case.

Let $G_{K}$ be the analytic subgroup of $G$ corresponding to $\mathfrak{R}_{K, 0} . G_{K}$ is compact and simply connected. Hence there is a 1-1 correspondence between representations of $G_{K}$ and those of $\mathfrak{\Omega}_{K, 0}$ (and therefore of $\mathfrak{\Omega}_{K}$ ). Hence for any $\Lambda \in \mathrm{P}_{K}$, $\mathfrak{D}_{\Lambda}$ can also be regarded as an equivalence class of representations of $G_{K}$. For 
any $\Lambda \in \mathrm{P}_{K}$ we denote by $V_{\Lambda}$ the set of all elements $\psi \in V$ such that either $\psi=0$ or the subspace $U$ spanned by all vectors of the form $\pi(g) \psi\left(g \in G_{K}\right)$ is finite-dimensional and the representation of $G_{K}$ induced on $U$ is fully reducible into components each of which belongs to $\mathfrak{D}_{\mathbf{\Lambda}}$. Put $V_{\mathbf{\Lambda}}^{0}=V_{\mathbf{1}} \cap V_{\mathbf{\Lambda}}$ and $V^{0}$ $=\sum_{\Lambda} V_{\Lambda}^{0}$. It can be shown (Mautner [11]) that $V^{0}$ is dense in $V$.

Choose a fundamental system $\left\{\alpha_{1}, \cdots, \alpha_{l}\right\}$ of roots of $\mathfrak{R}_{K}$ with respect to $\mathfrak{h}_{K}$. For any root $\alpha$ we define the corresponding element $H_{\alpha} \in \mathfrak{h}_{K}$ as before. We may again suppose that $\alpha\left(H_{\alpha}\right)=2$. Put $H_{i}=H_{\alpha_{i}}, 1 \leqq i \leqq l$. We note that $(-1)^{1 / 2} H_{\alpha} \in \mathfrak{h}_{K, 0}$ for every root $\alpha$.

Let $\pi$ be a representation of $G$ on $V$ with the character $\chi$. Since $V^{0}$ is dense in $V, V_{\Lambda_{0}}^{0} \neq\{0\}$ for some $\Lambda_{0} \in \mathrm{P}_{K}$. Hence $\mathfrak{D}_{\Delta_{0}}$ occurs in $\pi(\mathfrak{B})$. Therefore from Theorem $6, \chi=\chi_{\lambda} \times \chi_{-\mu}^{-}$where $\lambda-\mu$ is an integral function on $\mathfrak{h}_{K}$. The following theorem shows that the converse is also true.

THEOREM 7. Let $\lambda$ and $\mu$ be linear functions on $\mathfrak{h}_{K}$ such that $\lambda-\mu$ is integral. Then we can find a representation $\pi$ of $G$ on a Hilbert space such that $\pi$ has the character $\chi_{\lambda} \times \chi_{-\mu}^{-}$. Moreover if $\lambda+\bar{\lambda}$ is integral, we can find a unitary representation of $G$ with the character $\chi_{\lambda} \times \chi_{\bar{\lambda}}$.

For the proof of this theorem we follow a method which is due to Gelfand and Naimark [7]. Let $P$ be the set of all positive roots of $\mathfrak{R}_{K}$. We can choose elements $U_{\alpha}, V_{\alpha}(\alpha \in P)$ in $\Omega_{K, 0}$ such that $U_{\alpha}+(-1)^{1 / 2} V_{\alpha}=X_{\alpha} \neq 0$ and $U_{\alpha}-(-1)^{1 / 2} V_{\alpha}=X_{-\alpha} \neq 0$ are root elements corresponding to the roots $\alpha$ and $-\alpha$ respectively. Then the elements $(-1)^{1 / 2} H_{i}=H_{i}^{\prime}, 1 \leqq i \leqq l, U_{\alpha}, V_{\alpha}(\alpha \in P)$ form a base for $\mathfrak{l}_{K, 0}$. We define the linear mappings $\Gamma, \gamma$, and $\gamma^{+}$as in Part II (pp. 52-53) and put

$$
\begin{aligned}
W_{\alpha} & =U_{\alpha}-\Gamma\left(V_{\alpha}\right), \quad W_{\alpha}^{\prime}=V_{\alpha}+\Gamma\left(U_{\alpha}\right), \\
W_{-\alpha} & =U_{\alpha}+\Gamma\left(V_{\alpha}\right), \quad W_{-\alpha}^{\prime}=V_{\alpha}-\Gamma\left(U_{\alpha}\right), \\
Z_{\alpha} & =\gamma\left(X_{\alpha}\right)=\left(W_{\alpha}+(-1)^{1 / 2} W_{\alpha}^{\prime}\right) / 2, \\
Z_{-\alpha} & =\gamma\left(X_{-\alpha}\right)=\left(W_{-\alpha}-(-1)^{1 / 2} W_{-\alpha}^{\prime}\right) / 2, \\
Z_{\alpha}^{+} & =\gamma^{+}\left(X_{\alpha}\right)=\left(W_{-\alpha}+(-1)^{1 / 2} W_{-\alpha}^{\prime}\right) / 2, \\
Z_{-\alpha}^{+} & =\gamma^{+}\left(X_{-\alpha}\right)=\left(W_{\alpha}-(-1)^{1 / 2} W_{a}^{\prime}\right) / 2 .
\end{aligned}
$$

Let $\mathfrak{h}=\gamma\left(\mathfrak{h}_{K}\right)+\gamma^{+}\left(\mathfrak{h}_{K}\right), \mathfrak{h}_{0}=\mathfrak{h}_{K, 0}+\Gamma\left(\mathfrak{h}_{K, 0}\right)$. We have already identified (see p. 80) linear functions on $\mathfrak{h}$ with pairs $(\lambda, \mu)$ of linear functions on $\mathfrak{h}_{K}$. $\lambda$ being any linear function on $\mathfrak{h}_{K}$ we denote by $\lambda^{+}$and $\lambda^{-}$the linear functions on $\mathfrak{h}$ defined by the pairs $(\lambda, 0)$ and $(0, \lambda)$ respectively. Also we write $\lambda(\Gamma(H))$ $=-(-1)^{1 / 2} \lambda(H)\left(H \in \mathfrak{h}_{K}\right)$.

Let $\mathfrak{N}$ be the subspace of $\mathfrak{R}_{0}$ spanned by $W_{-\alpha}, W_{-\alpha}^{\prime}(\alpha \in P)$. Then $\mathfrak{N}$ is a nilpotent subalgebra of $\mathfrak{R}_{0}$. Put $\mathfrak{h}_{0}{ }^{*}=\Gamma\left(\mathfrak{h}_{K, 0}\right)$ and $\subseteq=\mathfrak{h}_{0}{ }^{*}+\mathfrak{N}$. Then

$$
\mathfrak{R}_{0}=\mathfrak{R}_{K, 0}+\mathfrak{h}_{0}^{*}+\mathfrak{N}=\mathfrak{R}_{K, 0}+\mathfrak{S}
$$


where all the sums are direct. Let $S, A$, and $N$ respectively be the analytic subgroups of $G$ corresponding to the subalgebras $\mathfrak{S}, \mathfrak{h}^{*}$, and $\mathfrak{N}$. Then it is known (see for example Iwasawa [10]) that the mapping $\Phi:(u, h, n) \rightarrow u h n$ $\left(u \in G_{K}, h \in A, n \in N\right)$ is a topological and analytic mapping of $G_{K} \times A \times N$ onto $G$. The tangent space of $G_{K} \times A \times N$ at any point $(u, h, n)$ is the Cartesian product of the tangent spaces of $G_{K}, A$, and $N$ at $u, h$, and $n$ respectively. But for a Lie group the tangent space at any point may be identified under left translation with the Lie algebra, which is the tangent space at the unit element (see Chevalley [6, Chap. IV]). Therefore we can, in a natural way, identify the tangent space of $G_{K} \times A \times N$ at any point $(u, h, n)$ with $\mathfrak{R}_{0}$. Let $d \Phi$ be the differential( $\left.{ }^{22}\right)$ of $\Phi$. Then for any $(u, h, n) \in G_{K} \times A \times N,(d \Phi)_{u, h, n}$ is a linear mapping of $\mathbb{R}_{0}$ into itself. Our object is to evaluate the Haar measure on $G$ in terms of the Haar measures on $G_{K}, A$, and $N$.

Let $\varphi, \psi, \chi$ be left invariant differential forms $(\neq 0)$ of degree $n, l$, and $n-l$ on $G_{K}, A$, and $N$ respectively. Here $n=\operatorname{dim} \mathfrak{R}_{K, 0}$ and $l=\operatorname{dim} \mathfrak{h}_{K, 0}$. Then we define a differential form $\xi$ of degree $2 n$ on $G_{K} \times A \times N$ as follows:

$$
\begin{aligned}
& \xi\left(U_{1}, \cdots, U_{n} ; H^{(1)}, \cdots, H^{(l)} ; X_{1}, \cdots, X_{n-l}\right) \\
&=\varphi\left(U_{1}, \cdots, U_{n}\right) \psi\left(H^{(1)}, \cdots, H^{(l)}\right) \chi\left(X_{1}, \cdots, X_{n-l}\right)
\end{aligned}
$$

for any $U_{i} \in \mathfrak{R}_{K, 0}, H^{(j)} \in \mathfrak{h}_{0}{ }^{*}, X_{k} \in \mathfrak{N}, 1 \leqq i \leqq n, 1 \leqq j \leqq l, 1 \leqq k \leqq n-l$. Let $\theta \neq 0$ denote a left invariant differential form of degree $2 n$ on $G$ and let $\delta \Phi$ be the mapping dual to $d \Phi$. Then $\delta \Phi$ maps $\theta$ on a differential form $\theta^{\prime}$ (of degree $2 n$ ) on $G_{K} \times A \times N$. If $\theta_{u, h, n}^{\prime}$ denotes the value of $\theta^{\prime}$ at any point $(u, h, n)$

$$
\begin{aligned}
\theta_{u, h, n}^{\prime}\left(U_{1}, \cdots, U_{n} ; H^{(1)},\right. & \left.\cdots, H^{(l)} ; X_{1}, \cdots, X_{n-l}\right) \\
& =\theta\left(U_{1}^{\prime}, \cdots, U_{n}^{\prime}, H^{\prime(1)}, \cdots, H^{\prime(l)}, X_{1}^{\prime}, \cdots, X_{n-l}^{\prime}\right)
\end{aligned}
$$

where $U_{i}, H^{(j)}$, and $X_{k}$ are as in (35) and $U_{i}^{\prime}, H^{\prime(j)}, X_{k}^{\prime}$ respectively are their transforms under $d \Phi_{u, h, n}$. Therefore we get

$$
\theta_{u, h, n}^{\prime}=c\left(\operatorname{det} d \Phi_{u, h, n}\right) \xi_{u, h, n}
$$

where $\xi_{u, h, n}$ is the value of $\xi$ at $(u, h, n)$ and $c$ is a fixed real number $\neq 0$. Let $f$ be a function on $G$ which is analytic around $u h n$. Put $F=f \bigcirc \Phi$. Then $F$ is analytic around $(u, h, n)$ on $G_{K} \times A \times N$. Let $X \in \mathfrak{N}$. Then

$$
\begin{aligned}
X F(u, h, n) & =\left\{\frac{d}{d t} F(u, h, n \exp t X)\right\}_{t=0} \\
& =\left\{\frac{d}{d t} f(u h n \exp t X)\right\}_{t=0} \\
& =X f(u h n) .
\end{aligned}
$$

(22) We use here and in the sequel the terminology of [6]. 
Similarly if $H \in \mathfrak{h}_{0}{ }^{*}$,

$$
\begin{aligned}
H F(u, h, n) & =\left\{\frac{d}{d t} F(u, h \exp t H, n)\right\}_{t=0} \\
& =\left\{\frac{d}{d t} f(u h \exp t H n)\right\}_{t=0} \\
& =\left\{\frac{d}{d t} f\left(u n h\left(\exp t \operatorname{Ad}\left(n^{-1}\right) H\right)\right)\right\}_{t=0}=\left(\operatorname{Ad}\left(n^{-1}\right) H\right) f(u h n)
\end{aligned}
$$

where $g \rightarrow$ Ad $(g)$ denotes the adjoint representation of $G$, so that

$$
g(\exp W) g^{-1}=\exp (\operatorname{Ad}(g) W)
$$

for any $W \in \mathfrak{R}_{0}$ and $g \in G$. Finally if $U \in \mathfrak{R}_{K, 0}$,

$$
U F(u, h, n)=\left\{\frac{d}{d t} F(u \exp t U, h, n)\right\}_{t=0}=\left\{\operatorname{Ad}\left(n^{-1} h^{-1}\right) U\right\} f(u h n) .
$$

These relations show that

$$
\begin{array}{llrl}
d \Phi_{u, h, n} U & =\operatorname{Ad}\left(n^{-1} h^{-1}\right) U & \left(U \in \mathfrak{R}_{K, 0}\right), \\
d \Phi_{u, h, n} H=\operatorname{Ad}\left(n^{-1}\right) H & \left(H \in \mathfrak{b}_{0}^{*}\right), \\
d \Phi_{u, h, n} X=X & (X \in \mathfrak{R}) .
\end{array}
$$

Let $W \rightarrow$ ad $W$ denote the adjoint representation of $\Omega_{0}$. Then it is easily seen that ad $X$ is nilpotent for all $X \in \Re$ and therefore det $\operatorname{Ad}(n)=1$ for all $n \in N$. Hence det $\left(d \Phi_{u, h, n}\right)=\operatorname{det} D$, where $D=\operatorname{Ad}(n) \mathrm{d} \Phi_{u, h, n}$. Now $\mathfrak{N}$ is invariant under $\operatorname{Ad}(n)$. Let $(\operatorname{Ad}(n))_{\mathfrak{R}}$ denote the restriction of $\operatorname{Ad}(n)$ on $\Re$. Then again we prove in the same way as above that $\operatorname{det}(\operatorname{Ad}(n))_{\Re}=1$. Also we notice from (38) that $\subseteq=\mathfrak{h}_{0}{ }^{*}+\mathfrak{N}$ is invariant under $D$. Let $D^{*}$ be the linear mapping of the factor space $\mathfrak{R}_{0} / \mathfrak{S}$ induced by $D$. Since det $(\operatorname{Ad}(n))_{\Re}=1$ it follows from (38) that $\operatorname{det} D=\operatorname{det} D^{*}$. Clearly $\subseteq$ is also invariant under Ad $\left(h^{-1}\right)$ and the linear mapping of $\Omega_{0} / \subseteq$ induced by $\operatorname{Ad}\left(h^{-1}\right)$ coincides with $D^{*}$. Now every $U \in \mathfrak{\Omega}_{K, 0}$ can be written uniquely in the form

$$
\begin{aligned}
U & =H^{\prime}+\sum_{\alpha \in P} 2 a_{\alpha} U_{\alpha}+\sum_{\alpha \in P} 2 b_{\alpha} V_{\alpha} \\
& =H^{\prime}+\sum_{\alpha \in P} a_{\alpha}\left(W_{\alpha}+W_{-\alpha}\right)+\sum_{\alpha \in P} b_{\alpha}\left(W_{\alpha}^{\prime}+W_{-\alpha}^{\prime}\right)
\end{aligned}
$$

where $H^{\prime} \in \mathfrak{h}_{K, 0}$ and $a_{\alpha}, b_{\alpha} \in R$. Now for any $H \in \mathfrak{h}_{0}{ }^{*}$ and $\alpha \in P$,

$$
\begin{aligned}
{\left[H, W_{\alpha}\right] } & =\alpha(H) W_{\alpha}, & {\left[H, W_{\alpha}^{\prime}\right] } & =\alpha(H) W_{\alpha}^{\prime}, \\
{\left[H, W_{-\alpha}\right] } & =-\alpha(H) W_{-\alpha}, & {\left[H, W_{-\alpha}^{\prime}\right] } & =-\alpha(H) W_{-\alpha}^{\prime},
\end{aligned}
$$


where $\alpha(H)=-(-1)^{1 / 2} \alpha\left(\Gamma^{-1}(H)\right)$ is real. Hence if $h=\exp H\left(H \in \mathfrak{h}_{0}^{*}\right)$,

$$
\begin{array}{rlr}
\operatorname{Ad}\left(h^{-1}\right) U=H^{\prime} & +\sum_{\alpha \in P} a_{\alpha}\left(e^{-\alpha(H)} W_{\alpha}+e^{\alpha(H)} W_{-\alpha}\right) & \\
& +\sum_{\alpha \in P} b_{\alpha}\left(e^{-\alpha(H)} W_{\alpha}^{\prime}+e^{\alpha(H)} W_{-\alpha}^{\prime}\right) & \\
\equiv H^{\prime} & +\sum_{\alpha \in P} a_{\alpha} e^{-\alpha(H)}\left(W_{\alpha}+W_{-\alpha}\right) & \\
& +\sum_{\alpha \in P} b_{\alpha} e^{-\alpha(H)}\left(W_{\alpha}^{\prime}+W_{-\alpha}^{\prime}\right) & \bmod \subseteq \\
\equiv H^{\prime} & +\sum_{\alpha \in P} 2 a_{\alpha} e^{-\alpha(H)} U_{\alpha}+\sum_{\alpha \in P} 2 b_{\alpha} e^{-\alpha(H)} V_{\alpha} & \bmod \subseteq .
\end{array}
$$

This shows that $\operatorname{det} D^{*}=\exp \left(-2 \sum_{\alpha \epsilon P} \alpha(H)\right)=e^{-4 \rho(H)}$. Hence

$$
\theta_{u, h, n}^{\prime}=c e^{-4 \rho(H)} \xi_{u, h, n} \quad\left(h=\exp H, H \in \mathfrak{h}_{0}^{*}\right)
$$

and it follows that the left invariant Haar measure on $G$ is given by

$$
e^{-4 \rho(H)} d u d h d n
$$

where $d u, d h, d n$ respectively are the left invariant Haar measures on $G_{K}, A$, and $N$ and $h=\exp H\left(H \in \mathfrak{h}_{0}^{*}\right)$.

Let $x \in G$ and $u \in G_{K}$. Then $x u=u_{x} h(x, u) n(x, u)$ where $u_{x} \in G_{K}, h(x, u)$ $\in A, n(x, u) \in N$. Since $A$ is simply connected there is a unique element $H(x, u) \in \mathfrak{h}_{0}{ }^{*}$ such that $h(x, u)=\exp H(x, u)$. Clearly $u_{x}, H(x, u)$, and $n(x, u)$ are continuous functions of $(x, u)$ and for a fixed $x, u \rightarrow u_{x}\left(u \in G_{K}\right)$ is a topological mapping of $G_{K}$ onto itself. Let $y=u h n\left(u \in G_{K}, h \in A, n \in N\right)$, and let $d y$ denote the left invariant Haar measure of $G$. Then for a fixed $x \in G$,

$$
d(x y)=d y=e^{-4 \rho(H)} d u d h d n .
$$

On the other hand

$$
\begin{aligned}
x y & =x u h n=u_{x} h(x, u) n(x, u) h n \\
& =u_{x} h(x, u) h\left(h^{-1} n(x, u) h n\right) .
\end{aligned}
$$

Since $\mathfrak{N}$ is an ideal in $\subseteq, N$ is an invariant subgroup of $S$. Hence $h^{-1} n(x, u) h$ $=n(x, u, h) \in N$. Also for fixed $(x, u), d(h(x, u) h)=d h$ and for fixed $(x, u, h)$, $d(n(x, u, h) n)=d n$ on account of the left invariance of the Haar measures. Hence

$$
e^{-4 \rho(H)} d u d h d n=d(x y)=e^{-4 \rho(H)-4 \rho(H(x, u))} d u_{x} d h d n
$$

and therefore

$$
d u_{x}=e^{4 \rho(B(x, u))} d u .
$$

Let $f$ be any function on $G_{K}$. For any $x \in G$ we define a new function $f^{x}$ on $G_{K}$ as follows: 


$$
f^{x}(u)=f\left(u_{x^{-1}}\right) .
$$

Let $L_{2}\left(G_{K}\right)$ be the Hilbert space consisting of all measurable functions $f$ on $G_{K}$ such that $\int_{G_{K}}|f(u)|^{2} d u<\infty$. Then if $f \in L_{2}\left(G_{K}\right)$,

$$
\int_{G_{K}}\left|f^{x}(u)\right|^{2} d u=\int_{G_{K}}|f(u)|^{2} d u_{x}=\int_{G_{K}}|f(u)|^{2} e^{4 \rho(\boldsymbol{H}(x, u))} d u<\infty
$$

since the function $e^{4 \rho(H(x, u))}$, being continuous, is bounded on the compact set $G_{K}$. Notice that if $y \in G$,

$$
y x u=y u_{x} h(x, u) n=\left(u_{x}\right)_{y} h\left(y, u_{x}\right) h(x, u) n^{\prime}
$$

where $n, n^{\prime} \in N$. Hence $u_{y x}=\left(u_{x}\right)_{y}$ and $H(u, y x)=H\left(y, u_{x}\right)+H(x, u) . \nu$ being any linear function on $\mathfrak{h}_{K}$ and $x \in G, f \in L_{2}\left(G_{K}\right)$, we define $\pi(x) f=f^{\prime} \in L_{2}\left(G_{K}\right)$ as follows

$$
f^{\prime}\left(u_{x}\right)=e^{\nu(\boldsymbol{H}(x, u))} f(u) \quad\left(u \in G_{K}\right) .
$$

Then by using the above relations it is easily verified that $\pi$ is a representation of $G$ on $L_{2}\left(G_{K}\right)$. We shall write $\pi(x) f(u)$ for $f^{\prime}(u)\left(u \in G_{K}\right)$.

For any $x \in G$ and $W \in \Omega_{0}$ put $W^{x}=\operatorname{Ad}(x) W$. Then we have the following lemma.

Lemma 45. Let $u \in G_{K}$ and $\varphi \in L_{2}\left(G_{K}\right)$. Then if $x \in u N u^{-1}, \pi(x) \varphi(u)=\varphi(u)$. Moreover if $x=\exp H^{u}\left(H \in \mathfrak{h}_{0}^{*}\right), \pi(x) \varphi(u)=e^{\nu(H)} \varphi(u)$.

Let $x=u n u^{-1}(n \in N)$. Then $x u=u n$. Hence $u_{x}=u$ and $H(x, u)=0$. Therefore $\pi(x) \varphi(u)=\pi(x) \varphi\left(u_{x}\right)=\varphi(u)$. Now let $x=\exp H^{u}\left(H \in \mathfrak{h}_{0}^{*}\right)$. Then $x u$ $=u \exp H$. Hence $u_{x}=u, H(x, u)=H$. Hence $\pi(x) \varphi(u)=\pi(x) \varphi\left(u_{x}\right)=e^{\nu(H)} \varphi(u)$.

Let $A_{K}$ be the analytic subgroup of $G_{K}$ corresponding to the subalgebra $\mathfrak{h}_{K, 0}$ of $\mathfrak{R}_{K, 0}$.

Lemma 46. Let $u \in G_{K}$ and $h \in A_{K}$. Then for any $x \in G$,

$$
(u h)_{x}=u_{x} h, \quad H(x, u h)=H(x, u) .
$$

Notice that elements of $A_{K}$ and $A$ commute and $\mathfrak{N}$ is invariant under ad $H$ for all $H \in \mathfrak{h}_{K, 0}$. Hence $h^{-1} N h \subset N$. But

$$
x u h=u_{x} h(x, u) n h=u_{x} h(x, u) h\left(h^{-1} n h\right)
$$

where $n \in N$. Hence

$$
u_{x}=u_{x} h, \quad h(x, u h)=h(x, u) .
$$

This proves the lemma.

Let $\Lambda$ be any integral function on $\mathfrak{h}_{K}$. Then for some $\Lambda_{0} \in \mathrm{P}_{K},-\Lambda$ is a weight of $\mathfrak{D}_{\Lambda_{0}}$. Since every finite-dimensional irreducible representation of $G_{K}$ occurs in the right regular representation of $G_{K}$ we can find a continuous function $\psi \neq 0$ on $G_{K}$ such that $\psi$ transforms according to $\mathfrak{D}_{\Lambda_{0}}$ under the right 
regular representation of $G_{K}$ and belongs to the weight $-\Lambda$. Then $\psi(u \exp H)$ $=e^{-\Lambda(H)} \psi(u)$ for any $u \in G_{K}$ and $H \in \mathfrak{h}_{K, 0}$.

Lemma 47. Let $x \in G, u \in G_{K}$, and $H \in \mathfrak{h}_{K, 0 .}$. Then

$$
\pi\left(\exp H^{u}\right) \pi(x) \psi(u)=e^{\Delta(H)} \pi(x) \psi(u) .
$$

Now

$$
\pi\left(\exp H^{u}\right) \pi(x) \psi(u)=\pi(x) \psi\left(\left(\exp \left(-H^{u}\right)\right) u\right)=\pi(x) \psi\left(u h^{-1}\right)
$$

where $h=\exp H$. But from Lemma 46,

$$
\begin{aligned}
\pi(x) \psi\left(u h^{-1}\right) & =\exp \left(\nu\left(H\left(x,\left(u h^{-1}\right)_{x^{-1}}\right)\right) \psi\left(\left(u h^{-1}\right)_{x^{-1}}\right)\right. \\
& =\exp \left(\nu\left(H\left(x, u_{x^{-1}} h^{-1}\right)\right)\right) \psi\left(u_{x^{-1}}\right) e^{\Delta(H)} \\
& =\exp \left(\nu\left(H\left(x, u_{x^{-1}}\right)\right)\right) \psi\left(u_{x^{-1}}\right) e^{\Delta(B)} \\
& =e^{\Delta(H)} \pi(x) \psi(u) .
\end{aligned}
$$

Hence the result.

LEMмa 48. Let $V_{\psi}$ be the set of all $\varphi \in L_{2}(G)$ which are of the form

$$
\varphi=\int_{G} f(x) \pi(x) \psi d x \quad\left(f \in C_{c}^{\infty}\right) .
$$

Then $\pi(z) \varphi=\chi(z) \varphi \quad\left(z \in \mathcal{B}, \varphi \in V_{\psi}\right)$ where $\chi=\chi_{\lambda} \times \chi_{-\mu}^{-}$and $\lambda=(\Lambda+\nu) / 2$, $\mu=(\nu-\Lambda) / 2$.

It is clear that $V_{\psi}$ is invariant under $\pi(\mathfrak{B})$ and $\pi(G)$. Also it follows from Lemmas 45 and 47 that

$$
\begin{array}{rlrl}
\pi\left(\exp t W_{-\alpha}^{u}\right) \varphi(u) & =\pi\left(\exp t W_{-\alpha}^{\prime u}\right) \varphi(u)=\varphi(u) & (\alpha \in P), \\
\pi\left(\exp t H^{u}\right) \varphi(u)=e^{t \nu(H)} \varphi(u) & \left(H \in \mathfrak{h}_{0}^{*}\right), \\
\pi\left(\exp t H^{u}\right) \varphi(u)=e^{t \Delta(H)} \varphi(u) & \left(H \in \mathfrak{h}_{0, K}\right)
\end{array}
$$

for any $\varphi \in V_{\psi}$ and $u \in G_{K}$. Since $V_{\psi}$ is invariant under $\pi(\mathfrak{B})$, the same relations hold true if we replace $\varphi$ by $\pi(b) \varphi(b \in \mathfrak{B})$. Hence it is clear that

$$
\begin{array}{rlrl}
\pi\left(W_{-\alpha}^{u} b\right) \varphi(u) & =\pi\left(W_{-\alpha}^{\prime u} b\right) \varphi(u)=0 & & (\alpha \in P), \\
\pi\left(H^{u} b\right) \varphi(u) & =\nu(H) \pi(b) \varphi(u) & \left(H \in \mathfrak{h}_{0}^{*}\right), \\
\pi\left(H^{u} b\right) \varphi(u) & =\Lambda(H) \pi(b) \varphi(u) & \left(H \in \mathfrak{h}_{0, K}\right) .
\end{array}
$$

Now for any $Z=W_{1}+(-1)^{1 / 2} W_{2} \in \mathfrak{R}\left(W_{1}, W_{2} \in \mathcal{R}_{0}\right)$ put

$$
Z^{x}=W_{1}^{x}+(-1)^{1 / 2} W_{2}^{x} \quad(x \in G) .
$$


Then from (33) it follows that

$$
\begin{aligned}
\pi\left(Z_{-\alpha}^{u} b\right) \varphi(u) & =\pi\left(Z_{\alpha}^{+u} b\right) \varphi(u)=0 & & (\alpha \in P) \\
\pi\left(H^{u} b\right) \varphi(u) & =\left(\lambda^{+}(H)-\mu^{-}(H)\right) \pi(b) \varphi(u) & & (H \in \mathfrak{h})
\end{aligned}
$$

where $\lambda=(\Lambda+\nu) / 2, \mu=(\nu-\Lambda) / 2$. For any $x \in G$, the mapping $Z \rightarrow Z^{x}(Z \in \mathbb{R})$ is an automorphism of $\mathbb{R}$ which can be extended uniquely to an automorphism of $\mathfrak{B}$ which we denote by $b \rightarrow b^{x}(b \in \mathfrak{B})$. It is clear that if $z \in \mathfrak{Z}, z^{x}=z$. Let $\mathfrak{S}$ be the subalgebra of $\mathfrak{B}$ generated by 1 and $\mathfrak{h}=\gamma\left(\mathfrak{h}_{K}\right)+\gamma^{+}\left(\mathfrak{h}_{K}\right)$. Let $\beta$ denote the isomorphism of $\mathfrak{S}$ with the ring $C[x, y]$ of polynomials in the indeterminates $\left(x_{1}, \cdots, x_{l}, y_{1}, \cdots, y_{l}\right)$ with coefficients in $C$ given by

$$
\beta\left(\gamma\left(H_{1}^{m_{1}} \cdots H_{l}^{m_{l}}\right) \gamma^{+}\left(H_{1}^{n_{1}} \cdots H_{l}^{n_{l}}\right)\right)=x_{1}^{m_{1}} \cdots x_{l}^{m_{l}} y_{1}^{n_{1}} \cdots y_{l}^{n_{l}}, \quad m_{i}, n_{i} \geqq 0 .
$$

Then if we take( $\left.{ }^{23}\right)\left(\alpha_{i}, 0\right),\left(0,-\alpha_{i}\right), 1 \leqq i \leqq l$, as a fundamental system of roots of $\mathfrak{Z}$ with respect to $\mathfrak{h}$, for every $z \in \mathfrak{Z}$ we can find a unique element $f_{z}(x, y) \in C[x, y]$ such that

$$
z-\beta^{-1}\left(f_{z}(x, y) \in \sum_{\alpha \in P} \mathfrak{B} Z_{\alpha}+\sum_{\alpha \in P} \mathfrak{B} Z_{-\alpha}^{+}\right.
$$

Hence

$$
z-\beta^{-1}\left(f_{z}(x, y)\right)=\sum_{\alpha \in P} a_{-\alpha} Z_{\alpha}+\sum_{\alpha \in P} b_{\alpha} Z_{-\alpha}^{+} \quad\left(a_{-\alpha}, b_{\alpha} \in \mathfrak{B}\right) .
$$

Since the left-hand side is of rank zero, we may suppose that $a_{-\alpha}$ is of rank $(-\alpha, 0)$ and $b_{\alpha}$ is of rank $(0, \alpha)$ with respect to $\mathfrak{h}$. Then we can show exactly as in the proof of Lemma 3, that $a_{-\alpha} \in \sum_{\alpha \in P} Z_{-\alpha} \mathfrak{B}, b_{\alpha}=\sum_{\alpha \in P} Z_{\alpha}^{+} \mathfrak{B}$. Hence

$$
z-\beta^{-1}\left(f_{z}(x, y)\right)=\sum_{\alpha \in P} Z_{-\alpha} c_{\alpha}+\sum_{\alpha \in P} Z_{\alpha}^{+} d_{\alpha} \quad\left(c_{\alpha}, d_{\alpha} \in \mathfrak{B}\right)
$$

and therefore

$$
z=z^{u}=\left(\beta^{-1}\left(f_{z}(x, y)\right)\right)^{u}+\sum_{\alpha \in P} Z_{-\alpha}^{u} c_{\alpha}^{u}+\sum_{\alpha \in P}\left(Z_{\alpha}^{+}\right)^{u} d_{\alpha}^{u} \quad\left(u \in G_{K}\right) .
$$

But then it follows from (41) that

$$
\pi(z) \varphi(u)=f_{z}(\lambda,-\mu) \varphi(u) .
$$

Since this is true for all $u \in G_{K}$,

$$
\pi(z) \varphi=f_{z}(\lambda,-\mu) \varphi .
$$

But it is clear that $f_{z}(\lambda,-\mu)=\chi(z)$ where $\chi=\chi_{\lambda} \times \chi_{-\mu}^{-}$. Hence the lemma is proved.

${ }^{(23)}$ As already mentioned earlier, we identify linear functions on $\mathfrak{h}$ with pairs of linear function on $\mathfrak{h}_{\boldsymbol{K}}$. 
The next two lemmas are due to Mautner. We use the usual terminology of Hilbert space. If $Q$ is an operator with a dense domain we denote by $Q^{*}$ its adjoint. Also if $Q_{1}, Q_{2}$ are two operators we say that $Q_{1} \subset Q_{2}$ if the domain $D_{1}$ of $Q_{1}$ is contained in the domain of $Q_{2}$ and $Q_{1}$ and $Q_{2}$ coincide on $D_{1}$.

Lemмa 49. Let $Q_{0}$ and $Q$ be two operators on a Hilbert space $V$ such that $Q_{0}$ and $Q^{*}$ are densely defined and $Q_{0} \subset Q$. Then if $Q_{0}$ is bounded so also is $Q$.

Since $Q_{0} \subset Q$ and $Q_{0}$ is bounded, $Q^{*} \subset Q_{0}{ }^{*}$ and $Q_{0}{ }^{*}$ is also bounded. Hence $Q^{*}$ and therefore $Q^{* *}$ is bounded. But $Q^{* *} \supset Q$. Therefore $Q$ is also bounded.

Lемма 50. Let $\pi$ be a representation of $G$ on a Hilbert space $V$. Then for any $b \in \mathfrak{B},(\pi(b)) *$ has a dense domain.

Let $V_{1}^{*}$ denote the set of all vectors $\varphi$ in $V$ of the form

$$
\varphi=\int_{G} f(g) \pi^{*}(g) \psi d g \quad\left(f \in C_{c}^{\infty}(G), \psi \in V\right)
$$

where $\pi^{*}(g)$ is the adjoint of $\pi(g)$. Also for any $X \in \mathfrak{R}_{0}$ and $f \in C_{c}^{\infty}(G)$ put

$$
\left(X^{*} f\right)(g)=\left\{\frac{d}{d t} f(g \exp t X)\right\}_{t=0} .
$$

Now

$$
\begin{aligned}
\pi^{*}(\exp (-t X)) \varphi & =\int_{G} f(g) \pi^{*}(g \exp (-t X)) \psi d g \\
& =\int_{G} f(g \exp t X) \pi^{*}(g) \psi d g
\end{aligned}
$$

since the Haar measure on $G$ is both left and right invariant. Hence

$$
\operatorname{Lim}_{t \rightarrow 0} \frac{1}{t}\left\{\pi^{*}(\exp t X) \varphi-\varphi\right\}=-\int_{G} X^{*} f(g) \pi^{*}(g) \psi d g \in V_{1}^{*} .
$$

From this it is clear that for any $X \in \Omega_{0},(\pi(X))^{*}$ is defined on $V_{1}^{*}$ and leaves it invariant. Hence it follows immediately that for any $b \in \mathfrak{B},(\pi(b))^{*}$ is defined on $V_{1}^{*}$. Since $V_{1}^{*}$ is dense in $V$ the assertion follows.

Now we return to the notation of Lemma 48 . Let $U$ be the closure of $V_{\psi}$. Then $U$ is clearly invariant under $\pi(G)$. Let $\pi_{0}$ be the representation of $G$ induced on $U$. Let $U_{1}$ be the Garding subspace of $U$.

LEMma 51. $\pi(z) \varphi=\chi(z) \varphi$ for any $z \in B$ and $\varphi \in U_{1}$. Hence $\pi_{0}$ has the character $\chi=\chi_{\lambda} \times \chi_{-\mu}^{-}$.

Clearly $V_{\psi} \subset U_{1}$. For any fixed $z \in ß$ let $Q_{0}$ and $Q$ be the operators in $U$ with the domains $V_{\psi}$ and $U_{1}$ respectively such that $Q_{0} \varphi=\pi(z) \varphi\left(\varphi \in V_{\psi}\right)$ 
and $Q \varphi=\pi(z) \varphi\left(\varphi \in U_{1}\right)$. Then $Q_{0} \subset Q$ and $Q_{0}$ is densely defined. Moreover by applying Lemma 46 to the representation $\pi_{0}$ of $G$ on $U$ we see that $Q^{*}$ is also densely defined. On the other hand we know from Lemma 48 that $Q_{0} \varphi$ $=\chi(z) \varphi\left(\varphi \in V_{\psi}\right)$. Hence $Q_{0}$ is bounded. Therefore from Lemma $46, Q$ is also bounded. Hence $Q_{0}$ and $Q$ have a unique common extension on $U$ which must be $\chi(z) I$ where $I$ is the unit operator. Therefore $Q \varphi=\chi(z) \varphi\left(\varphi \in U_{1}\right)$ and the lemma is proved.

We are now in a position to prove Theorem 7. Put $\lambda-\mu=\Lambda, \lambda+\mu=\nu$. Since $\Lambda$ is integral by hypothesis, the first assertion of the theorem follows from Lemma 51. Notice that if $\varphi \in L_{2}\left(G_{K}\right)$ and $x \in G$ we get from (40)

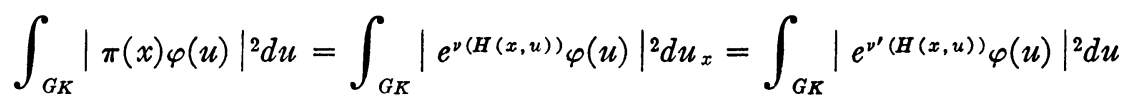

where $\nu^{\prime}=\nu+2 \rho$. Now suppose $\nu^{\prime}=(-1)^{1 / 2} \sigma$ where $\sigma$ is a real linear function on $\mathfrak{h}_{K}$ (that is, $\sigma\left(H_{i}\right), 1 \leqq i \leqq l$, are all real). Then $\nu^{\prime}(H)$ is pure imaginary for all $H \in \mathfrak{h}_{0}{ }^{*}$ and it is clear that the representation $\pi$ of $G$ on $L_{2}\left(G_{K}\right)$ is unitary. Moreover in this case $\lambda+\rho=\left(\Lambda+(-1)^{1 / 2} \sigma\right) / 2,-(\mu+\rho)=\left(\Lambda-(-1)^{1 / 2} \sigma\right) / 2$. Hence $-(\mu+\rho)=\bar{\lambda}+\rho$ and so from equation (23) of Part III, $\chi_{-\mu}^{-}=\chi_{\bar{\lambda}}$. Therefore $\pi_{0}$ is a unitary representation with the character $\chi_{\lambda} \times \chi_{\bar{\lambda}}$. Since $\Lambda$ can be any integral function and $\sigma$ any real function on $\mathfrak{h}_{K}$ it is obvious that $\lambda$ is arbitrary apart from the condition that $\lambda+\bar{\lambda}=\Lambda-2 \rho$ be integral. This completes the proof of Theorem 7 .

From the theory of representations of compact groups it follows that the function $\psi$ of Lemma 48 is a linear combination of the matrix elements of some representation of $G_{K}$ which belongs to $\mathfrak{D}_{\Lambda_{0}}$. Let $\mathfrak{D}_{\Lambda_{0}}^{*}=\mathfrak{D}_{\Lambda_{1}}\left(\Lambda_{1} \in \mathrm{P}_{K}\right)$ be the equivalence class of representations of $G_{K}$ which are dual to the representations in $\mathfrak{D}_{\Lambda_{0}}$. It is easily seen that under the left regular representation of $G_{K}, \psi$ transforms according to $\mathfrak{D}_{\Lambda_{1}}$. Hence $\mathfrak{D}_{\Lambda_{1}}$ occurs in the representation $\pi_{0}$ of Lemma 51 . Moreover it is clear that $-\Lambda$ is a weight of $\mathfrak{D}_{\Lambda_{0}}$ if and only if $\Lambda$ is a weight of $\mathfrak{D}_{\Lambda_{1}}$. Therefore we get the following extension of Theorem 7 .

THEOREM 8. Given any $\Lambda_{0} \in \mathrm{P}_{K}$ such that $\lambda-\mu$ is a weight of $\mathfrak{D}_{\Lambda_{0}}$ we can find a representation $\pi$ of $G$ on a Hilbert space such that $\pi$ has the character $\chi_{\lambda} \times \chi_{-\mu}^{-}$ and $\mathfrak{D}_{\Lambda_{0}}$ occurs in $\pi$. Similarly if $\lambda+\bar{\lambda}+2 \rho$ is a wcisht of $\mathfrak{D}_{\Lambda_{0}}$, we can find a unitary representation $\pi$ of $G$ with the character $\chi_{\lambda} \times \chi_{\bar{\lambda}}^{-}$such that $\mathfrak{D}_{\Lambda_{0}}$ occurs in $\pi$.

This is a sort of converse of Theorem 6 . We have mentioned earlier that every irreducible unitary representation $\pi$ of $G$ has a character $\chi$. It is not difficult to prove that in this case $\chi=\chi^{*}$ in the notation of Lemma 44. Taking into account the corollary to Lemma 44 , we see that Theorem 7 provides a partial answer to the problem of determining those characters of $\mathfrak{B}$ which correspond to some unitary representation of $G$ (see also Gelfand and Naimark [7]). 


\section{REFERENCES}

1. G. Birkhoff, Ann. of Math. vol. 38 (1937) pp. 526-532.

2. E. Cartan, Bull. Soc. Math. France vol. 41 (1913) pp. 53-96.

3. C. Chevalley, C. R. Acad. Sci. Paris vol. 227 (1948) pp. 1136-1138.

4. - Amer. J. Math. vol. 63 (1941) pp. 785-793.

5. - Ann. of Math. vol. 48 (1947) pp. 91-100.

6. - Theory of Lie groups, Princeton University Press, 1946.

7. I. M. Gelfand and M. A. Naimark, Doklady Akademii Nauk SSSR (N.S.) vol. 63 (1948) pp. 225-228.
8. L. Gårding, Proc. Nat. Acad. Sci. U.S.A. vol. 33 (1947) pp. 331-332.
9. Harish-Chandra, Ann. of Math. vol. 50 (1949) pp. 900-915.
10. K. Iwasawa, Ann. of Math. vol. 50 (1949) pp. 507-557.
11. F. I. Mautner, Ann. of Math. vol. 52 (1951) pp. 528-556.
12. H. Weyl, Math. Zeit. vol. 24 (1925) pp. 328-395.
13. - The classical groups, Princeton University Press, 1939.
14. E. Witt, J. Reine Angew. Math. vol. 177 (1937) pp. 152-160.

\section{HARVARD UNIVERSITY,}
Cambridge, Mass. 